\title{
Putting competing orders in their place near the Mott transition
}

\author{
Leon Balents, ${ }^{1}$ Lorenz Bartosch, ${ }^{2,3}$ Anton Burkov, ${ }^{1}$ Subir Sachdev, ${ }^{2}$ and Krishnendu Sengupta ${ }^{2}$ \\ ${ }^{1}$ Department of Physics, University of California, Santa Barbara, CA 93106-4030 \\ ${ }^{2}$ Department of Physics, Yale University, P.O. Box 208120, New Haven, CT 06520-8120 \\ ${ }^{3}$ Institut für Theoretische Physik, Universität Frankfurt, Postfach 111932, 60054 Frankfurt, Germany
}

(Dated: August 13, 2004)

\begin{abstract}
We describe the localization transition of superfluids on two-dimensional lattices into commensurate Mott insulators with average particle density $p / q(p, q$ relatively prime integers) per lattice site. For bosons on the square lattice, we argue that the superfluid has at least $q$ degenerate species of vortices which transform under a projective representation of the square lattice space group (a PSG). The formation of a single vortex condensate produces the Mott insulator, which is required by the PSG to have density wave order at wavelengths of $q / n$ lattice sites ( $n$ integer) along the principle axes; such a second-order transition is forbidden in the Landau-Ginzburg-Wilson framework. We also discuss the superfluid-insulator transition in the direct boson representation, and find that an interpretation of the quantum criticality in terms of deconfined fractionalized bosons is only permitted at special values of $q$ for which a permutative representation of the PSG exists. We argue (and demonstrate in detail in a companion paper: L. Balents et al., cond-mat/0409470) that our results apply essentially unchanged to electronic systems with short-range pairing, with the PSG determined by the particle density of Cooper pairs. We also describe the effect of static impurities in the superfluid: the impurities locally break the degeneracy between the $q$ vortex species, and this induces density wave order near each vortex. We suggest that such a theory offers an appealing rationale for the local density of states modulations observed by Hoffman et al, Science 295, 466 (2002), in scanning tunnelling microscopy (STM) studies of the vortex lattice of $\mathrm{Bi}_{2} \mathrm{Sr}_{2} \mathrm{CaCu}_{2} \mathrm{O}_{8+\delta}$, and allows a unified description of the nucleation of density wave order in zero and finite magnetic fields. We note signatures of our theory that may be tested by future STM experiments.
\end{abstract}

\section{INTRODUCTION}

One of the central debates in the study of cuprate superconductivity is on the nature of the electronic correlations in the 'underdoped' materials. At these low hole densities, the superconductivity is weak and appears only below a low critical temperature $\left(T_{c}\right)$, if at all. Nevertheless, there is no evidence of metallic behavior and an underlying Fermi surface of long-lived quasiparticles, as would be expected in a conventional BCS theory. This absence of Fermi liquid physics, and the many unexplained experimental observations, constitute key open puzzles of the field.

In very general and most basic terms, the passage from optimal doping to the underdoped region may be characterized by an evolution from conducting (even superconducting) to insulating behavior. Indeed, most thinking on the cuprates is informed by their proximity to a Mott insulating state, in which interaction-induced carrier localization is the primary driving influence. Many recent attempts to describe the underdoped region have, by contrast, focused on the presence or absence of conventional orders (magnetism, stripe, superconductivity, etc.) as a means of characterization. This focus has practical merit, such orders being directly measured by existing experimental probes. Theoretically, however, the immense panoply of competing potential orders reduces the predictive power of this approach, which moreover obscures the basic conducting to insulating transition in action in these materials. In this paper, we describe a theoretical approach in which Mott localization is "back in the driver's seat". Less colloquially, we characterize the behavior near to quantum critical points $(\mathrm{QCPs})$ whose dual "order parameter" primarily describes the emergence of an insulating state from a superconducting one. Remarkably, a proper quantum mechanical treatment of this Mott transition leads naturally to the appearance of competing conventional orders. The manner in which this occurs is described in detail below.

Among recent experiments, two classes are especially noteworthy for the issues to be discussed in our paper. Measurements of thermoelectric transport in a number of cuprates $^{1}$ have uncovered a large Nernst response for a significant range of temperatures above $T_{c}$. This response is much larger than would be expected in a Fermi liquid description. However, a model of vortex fluctuations over a background of local superfluid order does appear to lead to a satisfactory description of the data ${ }^{1}$. These experiments suggest a description of the underdoped state in terms of a fluctuating superconducting order parameter, $\Psi_{s c}$. Numerous such theories ${ }^{2}$ have been considered in the literature, under guises such as "preformed pairs" and "phase fluctuations". Intriguing new evidence in support of such a "bosonic Cooper pair" approach has been presented in recent work by Kapitulnik and collaborators ${ }^{3}$.

On the other hand, a somewhat different view of the underdoped state has emerged from a second class of experiments. Scanning tunnelling microscopy (STM) studies ${ }^{4-8}$ of $\mathrm{Bi}_{2} \mathrm{Sr}_{2} \mathrm{CaCu}_{2} \mathrm{O}_{8+\delta}$ and $\mathrm{Ca}_{2-x} \mathrm{Na}_{x} \mathrm{CuO}_{2} \mathrm{Cl}_{2}$ show periodic modulations in the electronic density of states, suggesting the appearance of a state with density wave order. Such density wave order can gap out portions of the Fermi surface, and so could be responsi- 
ble for onset of a spin-gap observed in the underdoped regime. However, the strength of the density wave modulations is quite weak, and so it is implausible that they produce the large spin gap. The precise microscopic nature of the density wave modulations also remains unclear, and they could represent spatial variations in the local charge density, spin exchange energy, and pairing or hopping amplitudes. Using symmetry considerations alone, all of these modulations are equivalent, because they are associated with observables which are invariant under time-reversal and spin rotations. It is useful, therefore, to discuss a generic density wave order, and to represent it by density wave order parameters $\rho_{\mathbf{Q}}$ which determine the modulations in the 'density' by

$$
\delta \rho(\mathbf{r})=\sum_{\mathbf{Q}} \rho_{\mathbf{Q}} e^{i \mathbf{Q} \cdot \mathbf{r}}
$$

where $\mathbf{Q} \neq 0$ extends over some set of wavevectors.

We will argue here that the apparent conflict between these two classes of experiments is neatly resolved by our approach. The strong vortex fluctuations suggest we examine a theory of superfluidity in the vicinity of a Mott transition. We will show that in a quantum theory of such a transition, which carefully accounts for the complex Berry phase terms, fluctuations of density wave order emerge naturally, and can become visible after pinning by impurities.

Within the conventional Landau-Ginzburg-Wilson (LGW) theory ${ }^{9,10}$, with the order parameters $\Psi_{s c}$ and $\rho_{\mathbf{Q}}$ at hand, a natural next step is to couple them to each other. Here, one begins by writing down the most general effective free energy consistent with the underlying symmetries. A key point is that $\Psi_{s c}$ and $\rho_{\mathbf{Q}}$ have non-trivial transformations under entirely distinct symmetries. The density wave order transforms under space group operations e.g. $T_{\mathbf{a}}$, translation by the lattice vector a

$$
T_{\mathbf{a}}: \rho_{\mathbf{Q}} \rightarrow \rho_{\mathbf{Q}} e^{i \mathbf{Q} \cdot \mathbf{a}} ; \Psi_{s c} \rightarrow \Psi_{s c},
$$

while $\Psi_{s c}$ transforms under the electromagnetic gauge transformation, $G$,

$$
G: \rho_{\mathbf{Q}} \rightarrow \rho_{\mathbf{Q}} \quad ; \quad \Psi_{s c} \rightarrow \Psi_{s c} e^{i \theta} .
$$

We could combine the two competing orders in a common 'superspin' order parameter (as in the ' $\mathrm{SO}(5)$ ' theory ${ }^{11}$ ), but neither of the symmetry operations above rotate between the two orders, and so there is no a priori motivation to use such a language. The transformations in Eqs. (1.2) and (1.3) place important constraints on the LGW functional of $\Psi_{s c}$ and $\rho_{\mathbf{Q}}$, which can be written as

$$
\mathcal{F}=\mathcal{F}_{1}\left[\Psi_{s c}\right]+\mathcal{F}_{2}\left[\rho_{\mathbf{Q}}\right]+\mathcal{F}_{3}\left[\Psi_{s c}, \rho_{\mathbf{Q}}\right] .
$$

Here $\mathcal{F}_{1}$ is an arbitrary functional of $\Psi_{s c}$ containing only terms which are invariant under Eq. (1.3), while $\mathcal{F}_{2}$ is a function of the $\rho_{\mathbf{Q}}$ invariant under space group operations like Eq. (1.2). These order parameters are coupled by $\mathcal{F}_{3}$, and the symmetry considerations above imply that $\mathcal{F}_{3}$ only contains terms which are products of terms already contained in $\mathcal{F}_{1}$ and $\mathcal{F}_{2}$. In other words, only the energy densities of the two order parameters couple to each other, and neither order has an information on the local 'orientation' of the other order. The crucial coupling between the orders is actually in an oscillatory Berry phase term, but this leaves no residual contribution in the continuum limit implicit in the LGW analysis. To leading order in the order parameters, the terms in Eq. (1.4) are

$$
\begin{aligned}
\mathcal{F}_{1}\left[\Psi_{s c}\right] & =r_{1}\left|\Psi_{s c}\right|^{2}+\ldots \\
\mathcal{F}_{2}\left[\rho_{\mathbf{Q}}\right] & =\sum_{\mathbf{Q}} r_{|\mathbf{Q}|}\left|\rho_{\mathbf{Q}}\right|^{2}+\ldots \\
\mathcal{F}_{3}\left[\Psi_{s c}, \rho_{\mathbf{Q}}\right] & =\sum_{\mathbf{Q}} v_{|\mathbf{Q}|}\left|\Psi_{s c}\right|^{2}\left|\rho_{\mathbf{Q}}\right|^{2}+\ldots
\end{aligned}
$$

The phase diagrams implied by the LGW functionals Eqs. (1.4) and (1.5) were determined by Liu and Fisher ${ }^{9}$, and are summarized here in Fig 1 . The transition between a state with only superconducting order $\left(\left\langle\Psi_{s c}\right\rangle \neq\right.$ $\left.0,\left\langle\rho_{\mathbf{Q}}\right\rangle=0\right)$ and a state with only density wave or$\operatorname{der}\left(\left\langle\Psi_{s c}\right\rangle=0,\left\langle\rho_{\mathbf{Q}}\right\rangle \neq 0\right)$ can follow one of 3 possible routes: (a) via a direct first order transition; (b) via an intermediate supersolid phase with both order parameters non-zero, which can be bounded by two second order transitions; and (c) via an intermediate "disordered" phase with both order parameters zero, which can also be bounded by two second order transitions. An important prediction of LGW theory is that there is generically no direct second order transition between the flanking states of Fig 1: such a situation requires fine-tuning of at least one additional parameter.

While the predictions of LGW theory in Fig 1 are appropriate for classical phase transitions at nonzero temperatures, they develop a crucial shortcoming upon a naive extension to quantum phase transitions at zero temperature. Importantly, the intermediate "disordered" phase in Fig 1 has no clear physical interpretation. At the level of symmetry, LGW theory informs us that this intermediate phase fully preserves electromagnetic gauge invariance and the space group symmetry of the lattice. Thinking quantum mechanically, this is not sufficient to specify the wavefunction of such a state. We could guess that this "disordered" state is a metallic Fermi liquid, which does preserve the needed symmetries. However, a Fermi liquid has a large density of states of low energy excitations associated with the Fermi surface, and these surely have to be accounted for near the quantum phase transitions to the other phases; clearly, such excitations are not included in the LGW framework. At a more sophisticated level, a variety of insulating states which preserve all symmetries of the Hamiltonian have been proposed in recent years ${ }^{12-14}$, but these have a subtle 'topological' order which is not contained in the LGW framework. Such topologically ordered states have global (and possibly local) low energy excitations and these have to be properly accounted for near quantum critical 


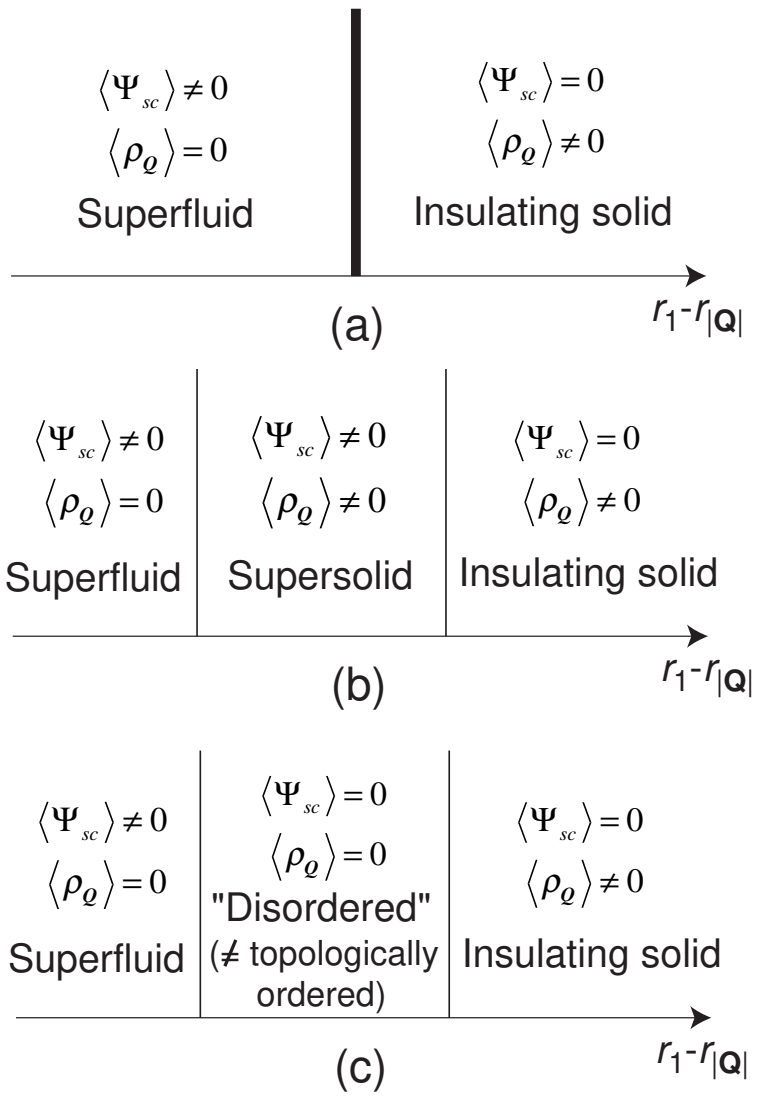

FIG. 1: LGW phase diagrams of the competition between the superconducting and density wave orders. The choice between the cases (a), (b), and (c) is made by the sign of $v_{|\mathbf{Q}|}$ and by the values of the higher order couplings in (1.5). The thick line is a first order transition, and the thin lines are second order transitions. The "disordered" phase of LGW theory preserves all symmetries but is not the "topologically ordered' phase which also preserves all symmetries: LGW theory does not predict the degeneracies of the latter state on topologically non-trivial spatial manifolds. It is believed there is no such "disordered" quantum state in the underlying quantum system at $T=0$.

points ${ }^{15}$; again, such excitations are entirely absent in the LGW framework. Thus the purported existence of a truly featureless intermediate "disordered" state in Fig 1c suggests serious shortcomings of the LGW description of multiple order parameters in quantum systems ${ }^{13-16}$.

Conceptually, it is useful to emphasize that the LGW approach to the conducting-insulating transition is quite the reverse of the Mott picture. Indeed, the above symmetry considerations do not at all refer to transport, or to localization of the carriers. To address such properties, a conventional application of the LGW method would be to introduce the $\rho_{\mathbf{Q}}$ and $\Psi_{s c}$ order parameters by a mean-field decoupling of a quantum model of bosons $^{17}$ or fermions ${ }^{18}$. These order parameters, once established, appear then as perturbations to the "quasiparticles" in the decoupled model. If $\rho_{\mathbf{Q}}$ becomes strong enough, the coincident potential barriers can localize the carriers. Thus in the LGW approach the Mott transition eventually arises out of the competing order, not viceversa.

In this paper, we will present an alternative approach to studying the interplay between the superfluid and density wave ordering in two spatial dimensions. Our analysis is based upon the recent work of Senthil et al. ${ }^{15}$ (and its precedents ${ }^{13,14,16}$ ), although we will present a different perspective. Also, the previous work ${ }^{15}$ was focused on systems at half-filling, while our presentation is applicable to lattice systems at arbitrary densities. One of the primary benefits of our approach is that the formalism naturally excludes the featureless 'disordered' states that invariably appear in the more familiar LGW framework. Our theory also makes it evident that the experimental observations of 'phase' fluctuations and pinned density wave order noted earlier are closely tied to each other, and, in principle, allows a compact, unified description of these experiments and also of the pinned density wave order around vortices in the superconductor ${ }^{19}$.

We will present our results here in the context of boson systems: specifically, in a general model of bosons hopping on a square lattice with short-range interactions. However, all the results of this paper apply essentially unchanged to electronic systems with short-range pairing. This is clearly the case for a toy model in which electrons experience a strong attractive interaction in the $s$-wave (or $d$-wave, though this is less obvious) channel, causing them to form tightly bound "molecular" Cooper pairs. Below the pair binding energy - i.e. the gap to unbound electron excitations - a description of the physics in terms of bosonic Cooper pairs clearly applies within such a model. Since we discuss universal physics near a bosonic superfluid to charge ordered transition, the theory here clearly describes such a transition from a short-range paired (also called the "strong pairing" regime/phase) superconductor to a density-wave ordered phase of any model, provided the electronic gap is maintained throughout the critical region. As we discuss below, a key parameter determining the character of the theories presented here is the number $f$, which for the lattice boson models is the average density of bosons per site of the square lattice in the Mott insulating state. Clearly, for electrons on the lattice with filling $1-\delta$, i.e. hole density $\delta$ relative to half-filling, the density of bosonic Cooper pairs is

$$
f=\frac{1-\delta}{2}
$$

We note in passing that the density of carriers in the superfluid proximate to the Mott insulator is allowed to be different from that of the Mott insulator, as elaborated in Section IIF.

These features will be explicitly demonstrated in a companion paper ${ }^{20}$, hereafter referred to as II. We argue in II that a convenient phenomenological model for capturing the spin $S=0$ sector of electrons near a superfluidinsulator transition is the doped quantum dimer model 
of the cuprate superconductors proposed by Fradkin and Kivelson $^{21}$. At zero hole concentration, the quantum dimer model ${ }^{22}$ generically has a Mott insulating ground state with valence bond solid (VBS) order ${ }^{16,23}$ (this order constitutes a particular realization of the generalized density wave order discussed above). Above some finite hole density, the dimer model has a superconducting ground state in which neutral observables preserve all lattice symmetries. (As we will discuss in II, the electronic pairing in this superconductor could have a $d$ - or $s$ (or other) wave character, depending upon microscopic details not contained in the dimer model). The evolution of the phase diagram between the insulating VBS state and the superconductor can occur via many possible sequences of transitions, all of which will be shown to be equivalent to those in the simpler boson models discussed in the present paper. A duality analysis of the doped dimer model presented in II directly gives the expected relation, Eq. (1.6). The paper II will also extend the dimer model to include fermionic $S=1 / 2$ excitations: this theory will be shown to have a close connection to other ${ }^{24} \mathrm{U}(1)$ or $\mathrm{SU}(2)$ gauge-theoretic "slave-particle" approaches to cuprate physics. Alternative formulations appropriate to long-range paired superconducting states (i.e. those with gapless nodal quasiparticle excitations) will also be discussed in II.

Let us turn, then, to a model of bosons hopping on a square lattice, with short-range interactions, ${ }^{61}$ and at a density $f$ per site of the square lattice. Our primary technical tool will be a dual description of this boson system using vortex degrees of freedom ${ }^{25-27}$. Briefly, the world lines of the bosons in three-dimensional spacetime are reinterpreted as the trajectories of vortices in a dual, classical, three-dimensional 'superconductor' with a dual 'magnetic' field oriented along the 'time' direction. The dual superconducting order parameter, $\psi$, is the creation and annihilation operator for vortices in the original boson variables. The density of the vortices in $\psi$ should equal the density of the bosons, and hence the dual 'magnetic' field acting on the $\psi$ has a strength of $f$ flux quanta per unit cell of the dual square lattice.

It is valuable to characterize the phases in Fig 1 in terms of the dual field $\psi$. It is easy to determine the presence/absence of superfluid order (which is associated with the presence/absence of a condensate in $\Psi_{s c}$ ). This has a dual relationship to the dual 'superconducting' order and is therefore linked to the absence/presence of a condensate in $\psi$ :

$$
\begin{aligned}
\text { Superfluid : }\langle\psi\rangle=0 \\
\text { Insulator : }\langle\psi\rangle \neq 0 .
\end{aligned}
$$

A condensate in $\psi$ implies a proliferation of vortices in $\Psi_{s c}$, and hence the loss of superfluid order. Strictly speaking, the loss of superfluidity does not necessarily require the appearance of a condensate of elementary vortices. It is sufficient that at least a composite of $n$ vortices condense, with $\left\langle\psi^{n}\right\rangle \neq 0$, where $n$ is a positive integer. The insulating states with $n>1$ and $\langle\psi\rangle=0$ have topological order ${ }^{12}$. For simplicity, we will not consider such insulating states here, although it is not difficult to extend our formalism to include such states and the associated multi-vortex condensates.

The characterization of the density wave order in terms of the vortex field, $\psi$, is more subtle, and a key ingredient in our analysis. We have noted that the field $\psi$ experiences a dual magnetic field of strength $f$ flux quanta per unit cell. Consequently, just as is familiar from the Hofstadter problem of electrons moving in a crystal lattice in the presence of a magnetic field ${ }^{28,29}$, the $\psi$ Hamiltonian transforms under a projective representation of the space group $^{30,31}$ or a PSG. A central defining property of a projective representation is that the operations $T_{x}, T_{y}$ of translation by one lattice site along the $x, y$ directions do not commute (as they do in any faithful representation of the space group), but instead obey

$$
T_{x} T_{y}=\omega T_{y} T_{x}
$$

where

$$
\omega \equiv e^{2 \pi i f}
$$

The PSG also contains elements corresponding to all other members of the square lattice space group. Among these are $R_{\pi / 2}^{\text {dual }}$ (rotation by angle $\pi / 2$ about a site of the dual lattice i.e. the sites of the lattice upon which the vortex field $\psi$ resides) and $I_{x}^{\text {dual }}, I_{y}^{\text {dual }}$ (reflections about the $x, y$ axes of the dual lattice); these obey ${ }^{32}$ group multiplication laws identical to those in the ordinary space group, e.g.

$$
\begin{aligned}
T_{x} R_{\pi / 2}^{\text {dual }} & =R_{\pi / 2}^{\text {dual }} T_{y}^{-1} \\
T_{y} R_{\pi / 2}^{\text {dual }} & =R_{\pi / 2}^{\text {dual }} T_{x} \\
\left(R_{\pi / 2}^{\text {dual }}\right)^{4} & =1
\end{aligned}
$$

All the elements of the PSG are obtained by taking arbitrary products of the elements already specified.

A useful and explicit description of this projective representation is obtained by focusing on the low energy and long wavelength fluctuations of $\psi$ at boson density

$$
f=\frac{p}{q}
$$

where $p$ and $q$ are relatively prime integers. We assume that the average density in the Mott insulator has the precise commensurate value in Eq. (1.11). In most of the paper we will also assume that the superfluid has the density value in Eq. (1.11), but it is not difficult to extend our analysis to allow the density of the superfluid (or supersolid) to stray from commensurate values to arbitrary incommensurate values: this will be discussed in Section II F. As we will review in Section II, the spectrum of $\psi$ fluctuations in the phase with $\langle\psi\rangle=0$ has $q$ distinct minima in the magnetic Brillouin zone. We can use these minima to define $q$ complex fields, $\varphi_{\ell}, \ell=0,1, \ldots, q-1$ 
which control the low energy physics. These $q$ vortex fields play a central role in all our analyses, as they allow us to efficiently characterize the presence or absence of superfluid and/or density wave order. In a convenient Landau gauge, the $q$ vortex fields can be chosen to transform under $T_{x}$ and $T_{y}$ as

$$
\begin{aligned}
& T_{x}: \varphi_{\ell} \rightarrow \varphi_{\ell+1} \\
& T_{y}: \varphi_{\ell} \rightarrow \varphi_{\ell} \omega^{-\ell} .
\end{aligned}
$$

Here, and henceforth, the arithmetic of all indices of the $\varphi_{\ell}$ fields is carried out modulo $q$, e.g. $\varphi_{q} \equiv \varphi_{0}$. The action of all the PSG elements on the $\varphi_{\ell}$ will be specified in Section II B; here we also note the important transformation under $R_{\pi / 2}^{\text {dual }}$

$$
R_{\pi / 2}^{\text {dual }}: \varphi_{\ell} \rightarrow \frac{1}{\sqrt{q}} \sum_{m=0}^{q-1} \varphi_{m} \omega^{-m \ell},
$$

which is a Fourier transform in the space of the $q$ fields. It is instructive to verify that Eqs. (1.12) and (1.13) obey Eqs. (1.8) and (1.10).

The existence of $q$ degenerate species of vortices in the superfluid is crucial to all the considerations of this paper. At first sight, one might imagine that quantum tunnelling between the different vortex species should lift the degeneracy, and we should only focus on the lowest energy linear combination of vortices. However, while such quantum tunnelling may lead to a change in the preferred basis, it does not lift the $q$-fold degeneracy. The degeneracy is imposed by the structure of the PSG, in particular the relation Eq. (1.8), and the fact that the minimum dimension of a PSG representation is $q$.

Using the transformations in Eqs. (1.12) and (1.13), we can now easily construct the required density wave order parameters $\rho_{\mathbf{Q}}$ as the most general bilinear, gaugeinvariant combinations of the $\varphi_{\ell}$ with the appropriate transformation properties under the square lattice space group. The density wave order parameters appear only at the wavevectors

$$
\mathbf{Q}_{m n}=2 \pi f(m, n),
$$

where $m, n$ are integers, and take the form

$$
\rho_{m n} \equiv \rho_{\mathbf{Q}_{m n}}=S\left(\left|\mathbf{Q}_{m n}\right|\right) \omega^{m n / 2} \sum_{\ell=0}^{q-1} \varphi_{\ell}^{*} \varphi_{\ell+n} \omega^{\ell m} .
$$

Here $S(Q)$ is a general 'form-factor' which cannot be determined from symmetry considerations, and has a smooth $Q$ dependence determined by microscopic details and the precise definition of the density operator. It is easy to verify that $\rho_{m n}^{*}=\rho_{-m,-n}$, and from Eqs. (1.12) and (1.13) that the space group operations act on $\rho_{m n}$ just as expected for a density wave order parameter

$$
\begin{aligned}
T_{x} & : \rho_{m n} \rightarrow \omega^{-m} \rho_{m n} \\
T_{y} & : \rho_{m n} \rightarrow \omega^{-n} \rho_{m n} \\
R_{\pi / 2}^{\text {dual }} & : \rho_{m n} \rightarrow \rho_{-n, m} .
\end{aligned}
$$

So by studying the bilinear combinations in Eq. (1.15), we can easily characterize the density wave order in terms of the $\varphi_{\ell}$. It is worth reiterating here that the $\rho_{m n}$ order parameters in Eq. (1.15) are generic density wave operators, and could, in principle, be adapted to obtain the actual density of either the bosons or the vortices. Indeed, as we noted above Eq. (1.1), we can obtain information on arbitrary observables invariant under spin rotations and time reversal, including the local density of states. The different specific observables will differ only in their values of the form factor $S(Q)$, which we do not specify in the present paper. Note also that because the $R_{\pi / 2}^{\text {dual }}$ rotation is about a dual lattice site, the last relation in Eq. (1.16) implies that the Fourier components $\rho_{\mathbf{Q}}$ are defined by taking the origin of the spatial co-ordinates on a dual lattice site. Upon performing the inverse Fourier transform from the $\rho_{m n}$ to real space (as in Eq. (2.26) below) the resulting density gives a measure (up to a factor of the unknown $S(Q)$ ) of the vortex density on the dual lattice sites, and of the boson density on the direct lattice sites. ${ }^{62}$

For completeness, we note that the characterizations in Eq. (1.7) of the superfluid order in terms of the vortex fields has an obvious expression in terms of the $\varphi_{\ell}$ :

$$
\begin{aligned}
\text { Superfluid }:\left\langle\varphi_{\ell}\right\rangle=0 \text { for every } \ell \\
\text { Insulator : }\left\langle\varphi_{\ell}\right\rangle \neq 0 \text { for at least one } \ell \text {. }
\end{aligned}
$$

The key relations in Eqs. (1.15) and (1.17) allow us to obtain an essentially complete characterization of all the phases of the boson model of interest here: this is a major reason for expressing the theory in terms of the vortex fields $\varphi_{\ell}$. Furthermore, the symmetry relations in Eqs. (1.12) and (1.13) impose strong constraints on the effective theory for the $\varphi_{\ell}$ fields whose consequences we will explore in Section II C. Indeed, even before embarking upon a study of this effective theory, we can already see that our present theory overcomes one of the key shortcomings of the LGW approach. We note from Eq. (1.17) that the non-superfluid phase has a condensate of one or more of the $\varphi_{\ell}$. Inserting these condensates into Eq. (1.15) we observe that $\left\langle\rho_{m n}\right\rangle \neq 0$ for at least one nonzero $\mathbf{Q}_{m n}$ i.e. the non-superfluid insulator has density wave order. Consequently the intermediate "disordered" phase in Fig 1c has been automatically excluded from the present theory. Instead, if the $\varphi_{\ell}$ condensate appears in a second-order transition (something allowed by the general structure of the theory), we have the possibility of a generic second-order transition from a superfluid phase to an insulator with density wave order, a situation which was forbidden by the LGW theory. ${ }^{63}$ We summarize the possible phase diagrams of the $\varphi_{\ell}$ theory in Fig 2 .

In addition to characterizing modulations in the "density" in terms of the $\rho_{m n}$, the $\varphi_{\ell}$ fields can also determine modulations in the local vorticity. This is a measure of circulating boson currents around plaquettes of the direct lattice ${ }^{33}$, and has zero average in all phases because time-reversal invariance is preserved. However, in the 


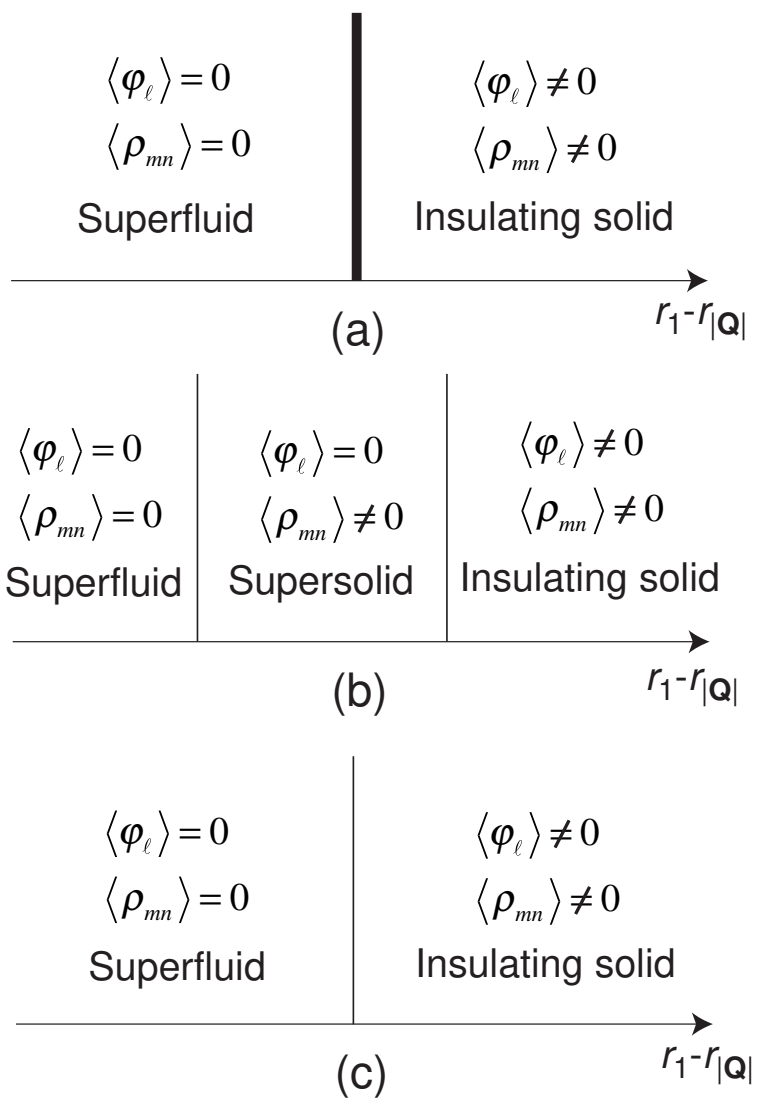

FIG. 2: Possible phase diagrams in the vortex theory of the interplay of superfluid and density wave order. Conventions are as in Fig 1. Notice that the objectionable "disordered" phase of the LGW theory has disappeared, and is replaced by a direct second order transition. Also, the first order transition in (a) is "fluctuation induced", i.e. unlike the LGW theory of Fig 1, the mean field vortex theory only predicts a second order transition, but fluctuations could induce a weak first order transition.

presence of an applied magnetic field, this constraint no longer applies. The magnetic field, of course, induces a net circulation of boson current, and the total number of vortices (or anti-vortices) is non-zero. However, using a reasoning similar to that applied above to the density, the PSG of the vortices implies there are also modulations in the vorticity at the $\mathbf{Q}_{m n}$ wavevectors. We can define a corresponding set of $V_{m n}$ which are Fourier components of the vorticity at these wavevectors. Details of this analysis, and explicit expressions for the $V_{m n}$ appear in Appendix A.

An explicit derivation of the above vortex theory of the Mott transition of the superfluid appear in Section II where we consider a model of bosons on the square lattice. Here we derive an effective action for the $q$ vortex species in Eqs. (2.19) and (2.21) which is invariant under the PSG, and which controls the phases and phase diagrams outlined above.

A complementary perspective is presented in Section III where we formulate the physics using the direct boson representation. In particular, following Senthil et $a l .^{15}$, we explore the possibility that the LGW forbidden superfluid-insulator transitions discussed above are associated with the boson fractionalization. The structure of the theory naturally suggests a fractionalization of each boson into $q$ components, each with boson number $1 / q$. However, the PSG places strong restrictions on the fractionalized boson theory. On the rectangular lattice, such a theory is consistent for all $q$ but only for certain insulating phases obtained for restricted parameter values. On the square lattice, with the additional $R_{\pi / 2}^{\text {dual }}$ element of the PSG, a fractionalized boson theory is permitted only for a limited set of $q$ values. Specifically, it is required that the $\varphi_{\ell}$ vortex fields transform under a permutative representation of the PSG. We define a permutative representation as one in which all group elements can be written as $\Lambda P$, where $\Lambda$ is a unitary diagonal matrix (i.e. a matrix whose only non-zero elements are complex numbers of unit magnitude on the diagonal), and $\mathrm{P}$ is a permutation matrix. For the representation defined in Eqs. (1.12) and (1.13), $T_{x}$ and $T_{y}$ are of this form, while $R_{\pi / 2}^{\text {dual }}$ is not. When a permutative representation exists, it is possible to globally unitarily transform the $\varphi_{\ell}$ fields to a new basis of $\zeta_{\ell}$ fields, which realize the permutative representation. For some range of parameters - values of the quantum tunnelling terms between the vortex flavors - one can see that the PSG is expected to be elevated at the critical point to include an emergent $U(1)^{q}$ (modulo the global $U(1)$ gauge invariance) symmetry of independent rotations of the $\zeta_{\ell}$ fields. This emergent symmetry is the hallmark of fractionalization, and corresponds to emergence of conserved $U(1)$ gauge fluxes in the direct representation. Such an emergent symmetry is clearly not generic, and this turns out to place quite a restrictive condition on the existence of a fractionalized $1 / q$ boson representation, as we discuss in detail in Section III, and in a number of appendices.

Section IV applies our theory to the STM observations of local density of states modulations in zero and nonzero magnetic field. A virtue of our approach is that it naturally connects density wave order with vorticity and allows a unified description of the experiments in zero and non-zero magnetic field. As we will discuss, there are several intriguing consequences of our approach, and we will analyze prospects for observing these in future STM experiments in Sections IV and V.

We briefly mention other recent works which have addressed related issues. Zaanen et al. ${ }^{34}$ have examined the superfluid to insulator transition from a complementary perspective: they focus on the dislocation defects of a particular insulating solid in the continuum, in contrast to our focus here on the vortex defects of the superfluid. We do consider the 'melting' of defects in the solid in Section III C, but the underlying lattice plays a crucial role in our considerations. In a work which appeared while our analysis was substantially complete, Tešanović ${ }^{35}$ has applied the boson-vortex duality to Cooper pairs and considered the properties of vortices in a dual 'magnetic' 
field.

\section{DUAL VORTEX THEORY OF BOSONS ON THE SQUARE LATTICE}

We consider an ordinary single-species boson model on the square lattice. The bosons are represented by rotor operators $\hat{\phi}_{i}$ and conjugate number operators $\hat{n}_{i}$ where $i$ runs over the sites of the direct square lattice. These operators obey the commutation relation

$$
\left[\hat{\phi}_{i}, \hat{n}_{j}\right]=i \delta_{i j}
$$

A simple boson Hamiltonian in the class of interest has the structure

$$
\begin{gathered}
\mathcal{H}=-t \sum_{i \alpha} \cos \left(\Delta_{\alpha} \hat{\phi}_{i}-2 \pi g_{i \alpha}\right)+\sum_{i} V\left(\hat{n}_{i}\right) \\
+\sum_{i \neq j} \Lambda_{i j} \hat{n}_{i} \hat{n}_{j}+\ldots
\end{gathered}
$$

where the interaction $V(\hat{n})$ has the on-site terms $V(\hat{n})=$ $-\bar{\mu} \hat{n}+U \hat{n}(\hat{n}-1) / 2$. The $\Lambda_{i j}$ are repulsive off-site interactions, and can also include the long-range Coulomb interaction. We will focus on the case of short-range $\Lambda_{i j}$, and note the minor modifications necessary for the longrange case. The general structure of our theory also permits a variety of exchange and ring-exchange terms, such as those in the studies of Sandvik et al. ${ }^{36}$. These off-site or ring exchange couplings are essential for our analysis, as they are needed to stabilize insulating phases of the bosons away from integer filling. Nevertheless, many aspects of our results are independent upon the particular form of these couplings; their specific form will only influence the numerical values of the non-linear couplings that appear in our phenomenological actions (such as those in Eq. (2.21)). The index $\alpha$ extends over the spatial directions $x, y$, while we will use indices $\mu, \nu, \lambda$ to extend over all three spacetime directions $x, y, \tau$. The symbol $\Delta_{\alpha}$ is a discrete lattice derivative along the $\alpha$ direction: $\Delta_{\alpha} \hat{\phi}_{i}=\hat{\phi}_{i+\alpha}-\hat{\phi}$ (and similarly for $\Delta_{\mu}$ ). We have also included a static external magnetic field represented by the vector potential $g_{i \alpha}$ for convenience. This is a uniform field which obeys

$$
\epsilon_{\mu \nu \lambda} \Delta_{\nu} g_{i \lambda}=h \delta_{\mu \tau}
$$

where $h$ is the strength of the physical magnetic field (which should be distinguished from the dual "magnetic" flux $f$ discussed in Section I).

\section{A. Dual lattice representation}

We proceed with a standard duality mapping, following the methods of Refs. 25-27 and the notational conventions of Ref. 37. We represent the partition function as Feynman integral over states at a large number of intermediate time slices, separated by the interval $\Delta \tau$. The intermediate states use a basis of $\hat{n}_{i}$ and $\hat{\phi}_{i}$ at alternate times. The hopping term in $\mathcal{H}$ acts between $\hat{\phi}_{i}$ eigenstates, and we evaluate its matrix elements by using the Villain representation

$$
\begin{gathered}
\exp \left(t \Delta \tau \cos \left(\Delta_{\alpha} \hat{\phi}_{i}-2 \pi g_{i \alpha}\right)\right) \\
\rightarrow \sum_{\left\{J_{i \alpha}\right\}} \exp \left(-\frac{J_{i \alpha}^{2}}{2 t \Delta \tau}+i J_{i \alpha} \Delta_{\alpha} \hat{\phi}_{i}-2 \pi i J_{i \alpha} g_{i \alpha}\right) .
\end{gathered}
$$

We have dropped an unimportant overall normalization constant, and will do so below without comment. The $J_{i \alpha}$ are integer variables residing on the links of the direct lattice, representing the current of the bosons.

After integrating over the $\phi_{i}$ on all sites and at all intermediate times, the partition function becomes

$$
\begin{aligned}
\mathcal{Z}=\sum_{\left\{J_{i \mu}\right\}} \exp \left(-\frac{1}{2 e^{2}} \sum_{i}\left(J_{i \mu}-H \delta_{\mu \tau}\right)^{2}\right. \\
\left.-\Delta \tau \sum_{i \neq j} \Lambda_{i j} J_{i \tau} J_{j \tau}-2 \pi i g_{i \mu} J_{i \mu}\right) \\
\times \prod_{i} \delta\left(\Delta_{\mu} J_{i \mu}\right)
\end{aligned}
$$

where $J_{i \mu} \equiv\left(n_{i}, J_{i x}, J_{i y}\right)$ is the integer-valued boson current in spacetime, $i$ now extends over the sites of the cubic lattice, and we have chosen $\Delta \tau$ so that $e^{2}=t \Delta \tau=$ $1 / U \Delta \tau$, and $H=\bar{\mu} / U+1 / 2$. We now solve the constraint in Eq. (2.5) by writing

$$
J_{i \mu}=\epsilon_{\mu \nu \lambda} \Delta_{\nu} A_{a \lambda}
$$

where $a$ labels sites on the dual lattice, and $A_{a \lambda}$ is an integer-valued gauge field on the links of the dual lattice. We promote $A_{a \mu}$ from an integer-valued field to a real field by the Poisson summation method, while "softening" the integer constraint with a fugacity $y_{v}$. It is convenient to make the gauge invariance of the dual theory explicit by introducing an angular field $\vartheta_{a}$ on the sites of the dual lattice, and mapping $2 \pi A_{a \mu} \rightarrow 2 \pi A_{a \mu}-\Delta_{\mu} \vartheta_{a}$. The operator $e^{i \vartheta_{a}}$ is then the creation operator for a vortex in the boson phase variable $\phi_{i}$. These transformations yield the dual partition function

$$
\begin{aligned}
\mathcal{Z}_{d}=\prod_{a} \int & d A_{a \mu} \int d \vartheta_{a} \exp ( \\
& -\frac{1}{2 e^{2}} \sum_{\square}\left(\epsilon_{\mu \nu \lambda} \Delta_{\nu} A_{a \lambda}-H \delta_{\mu \tau}\right)^{2} \\
& +y_{v} \sum_{a \mu} \cos \left(\Delta_{\mu} \vartheta_{a}-2 \pi A_{a \mu}\right) \\
& \left.-i h \sum_{a}\left(\Delta_{\tau} \vartheta_{a}-2 \pi A_{a \tau}\right)\right) .
\end{aligned}
$$


We have not explicitly displayed the $\Lambda_{i j}$ term above, and assumed it has been absorbed into a renormalized value of $e^{2}{ }^{64}$ This is the theory of a dual vortex boson represented by the angular rotor variable $\vartheta_{a}$, coupled to a dual gauge field $A_{a \mu}$. The Berry phase term proportional to $h$ is precisely the constraint that the rotor number variable (canonically conjugate to $\vartheta_{a}$ ) takes values which are integers plus $h$ (see Ref. 27): so there is a background density of vortices of $h$ per site which is induced by the magnetic field acting on the direct bosons.

The remainder of Section II will consider only the case $h=0$; the analog of the $h \neq 0$ case will appear later in the dimer model analyses of II.

We obtain the dual theory in its final form on the cubic lattice by replacing the field $e^{i \vartheta_{a}}$ by a "soft-spin" dual vortex field $\psi_{a}$, which yields

$$
\begin{aligned}
\mathcal{Z}_{d}=\prod_{a} \int & d A_{a \mu} \int d \psi_{a} \exp ( \\
& -\frac{1}{2 e^{2}} \sum_{\square}\left(\epsilon_{\mu \nu \lambda} \Delta_{\nu} A_{a \lambda}-H \delta_{\mu \tau}\right)^{2} \\
& +\frac{y_{v}}{2} \sum_{a \mu}\left[\psi_{a+\mu}^{*} e^{2 \pi i A_{a \mu}} \psi_{a}+\text { c.c. }\right] \\
& \left.-\sum_{a}\left[s\left|\psi_{a}\right|^{2}+\frac{u}{2}\left|\psi_{a}\right|^{4}\right]\right)
\end{aligned}
$$

The last line in Eq. (2.8) is the effective potential for the complex vortex field $\psi_{a}$. Increasing the parameter $s$ scans the system from the insulating solid at $s \ll 0$ to the superfluid at $s \gg 0$ 1.e. the system moves from right to left in the phase diagrams of Fig 2.

\section{B. Symmetries}

We will now present a careful analysis of the symmetries of Eq. (2.8), with the aim of deducing general constraints that must be obeyed by the low energy theory near the superfluid-to-insulator transition.

We begin in the superfluid regime with $s$ large, so that $\left\langle\psi_{a}\right\rangle=0$. The direct boson density is the $\tau$ component of the dual 'magnetic' flux $\epsilon_{\mu \nu \lambda} \Delta_{\nu} A_{\lambda}$, and for $s$ large in the action in Eq. (2.8) it is clear that the saddle point of the $A_{\mu}$ fluctuations occurs at $A_{a \mu}=\bar{A}_{a \mu}$ with $\epsilon_{\mu \nu \lambda} \Delta_{\nu} \bar{A}_{a \lambda}=$ $H \delta_{\mu \tau}$. We want this boson density to be the value $f$ in Eq. (1.11), and so we should choose $H=f$.

We now wish to examine the structure of $\psi_{a}$ fluctuations about this saddle point. Let us choose the Landau gauge with $\bar{A}_{a \tau}=\bar{A}_{a x}=0$ and

$$
\bar{A}_{a y}=f a_{x}
$$

here $a_{x}$ is the $x$ co-ordinate of the dual lattice $a$. We now need to determine the $\psi_{a}$ spectrum in a background $\bar{A}_{a \mu}$ field.

The basic symmetry operations are $T_{x}, T_{y}$, and $R_{\pi / 2}^{\text {dual }}$, introduced in Section I. The action of these operators on $\psi_{a} \equiv \psi\left(a_{x}, a_{y}\right)$ required to keep the Hamiltonian invariant is

$$
\begin{aligned}
T_{y} & : \psi\left(a_{x}, a_{y}\right) \rightarrow \psi\left(a_{x}, a_{y}-1\right) \\
T_{x} & : \psi\left(a_{x}, a_{y}\right) \rightarrow \psi\left(a_{x}-1, a_{y}\right) \omega^{a_{y}} \\
R_{\pi / 2}^{\text {dual }} & : \psi\left(a_{x}, a_{y}\right) \rightarrow \psi\left(a_{y},-a_{x}\right) \omega^{a_{x} a_{y}}
\end{aligned}
$$

Notice that Eqs. (1.8) and (1.10) are obeyed by the above.

It is also useful to collect the representation of Eq. (2.10) in momentum space. Implying that all momenta are reduced back to the extended Brillouin zone with momenta $-\pi<k_{x}, k_{y}<\pi$ we find

$$
\begin{aligned}
T_{y} & : \psi\left(k_{x}, k_{y}\right) \rightarrow \psi\left(k_{x}, k_{y}\right) e^{-i k_{y}} \\
T_{x} & : \psi\left(k_{x}, k_{y}\right) \rightarrow \psi\left(k_{x}, k_{y}-2 \pi f\right) e^{-i k_{x}} \\
R_{\pi / 2}^{\text {dual }} & : \psi\left(k_{x}, k_{y}\right) \rightarrow \\
& \frac{1}{q} \sum_{m, n=0}^{q-1} \psi\left(k_{y}+2 \pi n f,-k_{x}-2 \pi m f\right) \omega^{-m n}
\end{aligned}
$$

The most important $\psi_{a}$ fluctuations will be at momenta at which the spectrum has minima. It is not difficult to show from the above symmetry relations that any such minimum is at least $q$-fold degenerate: Let $|\Lambda\rangle$ be a state at a minimum of the spectrum. Because, the operator $T_{y}$ commutes with the Hamiltonian, this state can always be chosen to be an eigenstate of $T_{y}$, with eigenvalue $e^{-i k_{y}^{*}}$. Now the above relations imply immediately that $T_{x}|\Lambda\rangle$ is also an eigenstate of the Hamiltonian with $T_{y}$ eigenvalue $e^{-i k_{y}^{*} \omega^{-1}}$. Also, because the $T_{y}$ eigenvalue is distinct from that of $|\Lambda\rangle$, this state is an orthogonal eigenstate of the Hamiltonian. By repeated application of this argument, we obtain $q$ orthogonal eigenstates of the Hamiltonian whose $T_{y}$ eigenvalues are $e^{-i k_{y}^{*}}$ times integer powers of $\omega^{-1}$. Because we are working with the gauge of Eq. (2.9), our Hamiltonian couples momenta $\left(k_{x}, k_{y}\right)$ to momenta $\left(k_{x} \pm 2 \pi f, k_{y}\right)$. It is therefore advantageous to look at the spectrum in the reduced Brillouin zone with $-\pi / q<k_{x}<\pi / q$ and $-\pi<k_{y}<\pi$. For the nearestneighbor model under consideration here, the minima of the spectrum are then at the $q$ wavevectors $(0,2 \pi \ell p / q)$ with $\ell=0, \ldots q-1$. Let us label the eigenmodes at these wavevectors $\varphi_{\ell}$. We therefore have to write down the field theory in terms of these $q$ complex fields $\varphi_{\ell}$.

It is useful, especially when analyzing the influence of $R_{\pi / 2}^{\text {dual }}$, to make the above symmetry considerations explicit. In the extended Brillouin zone, for the nearestneighbor model under consideration here, let us label the $q^{2}$ states at the wavevectors $(2 \pi m p / q, 2 \pi n p / q)$ by $|m, n\rangle$. Then one of the minima of the spectrum corresponds to the state

$$
\left|\varphi_{0}\right\rangle=\sum_{m=0}^{q-1} c_{m}|m, 0\rangle
$$

where the $c_{m}$ are some complex numbers. Then, by operation of $T_{x}$ on $\left|\varphi_{0}\right\rangle$ we obtain the $q$ degenerate eigenstates 
as

$$
\left|\varphi_{\ell}\right\rangle=\sum_{m=0}^{q-1} c_{m} \omega^{-\ell m}|m, \ell\rangle
$$

Now let us consider the action of $R_{\pi / 2}^{\text {dual }}$ on the states in Eq. (2.13). Using Eq. (2.11), and after some simple changes of variables we obtain

$$
R_{\pi / 2}^{\mathrm{dual}}\left|\varphi_{\ell}\right\rangle=\frac{1}{q} \sum_{m, m^{\prime}, \ell^{\prime}=0}^{q-1} c_{m} \omega^{-\left(m^{\prime} \ell^{\prime}+\ell \ell^{\prime}-m m^{\prime}\right)}\left|m^{\prime}, \ell^{\prime}\right\rangle
$$

Now, because $R_{\pi / 2}^{\text {dual }}$ commutes with $H$, the right-handside of Eq. (2.14) must be a linear combination of the $\left|\varphi_{\ell}\right\rangle$ states in Eq. (2.13). The matrix elements of the rotation operator are then given by $\left\langle\varphi_{\ell^{\prime}}\left|R_{\pi / 2}^{\text {dual }}\right| \varphi_{\ell}\right\rangle=c \omega^{-\ell \ell^{\prime}}$, where $c=\frac{1}{q} \sum c_{m^{\prime}}^{*} c_{m} \omega^{m m^{\prime}}$ is independent of $\ell$ and $\ell^{\prime}$. For the nearest-neighbor model under consideration here, it can be easily checked that the $c_{m}$ 's are invariant under a Fourier transform such that $c=1 / \sqrt{q}$. Hence we find

$$
R_{\pi / 2}^{\mathrm{dual}}\left|\varphi_{\ell}\right\rangle=\frac{1}{\sqrt{q}} \sum_{\ell^{\prime}=0}^{q-1} \omega^{-\ell \ell^{\prime}}\left|\varphi_{\ell^{\prime}}\right\rangle
$$

Our discussion above has now established that the low energy vortex fields must have an action invariant under the transformations in Eqs. (1.12) and (1.13). In a similar manner we can also determine the transformations associated with the remaining elements of the square lattice space group. These involve the operations $I_{x}^{\text {dual }}$ and $I_{y}^{\text {dual }}$ which are reflections about the $x$ and $y$ axes of the dual lattice. Under these operations we find

$$
\begin{aligned}
& I_{x}^{\text {dual }}: \varphi_{\ell} \rightarrow \varphi_{\ell}^{*} \\
& I_{y}^{\text {dual }}: \varphi_{\ell} \rightarrow \varphi_{-\ell}^{*} .
\end{aligned}
$$

Finally it is interesting to consider the point inversion operator $I_{p}^{\text {dual }} \equiv\left(R_{\pi / 2}^{\text {dual }}\right)^{2}$, with

$$
I_{p}^{\mathrm{dual}}: \varphi_{\ell} \rightarrow \varphi_{-\ell} .
$$

As in the ordinary space group we have

$$
I_{p}^{\text {dual }}=I_{x}^{\text {dual }} I_{y}^{\text {dual }}=I_{y}^{\text {dual }} I_{x}^{\text {dual }} .
$$

\section{Continuum field theories}

We have established that fluctuations of the vortex fields about the saddle point of Eq. (2.8) in Eq. (2.9) transform under a projective representation of the square lattice space group which is defined by Eqs. (1.12), (1.13), and (2.16). In this section we will write down the most general continuum theory of the $\varphi_{\ell}$ fields which is invariant under these projective transformations. The action should include fluctuations in $A_{a \mu}$ about $\bar{A}_{a \mu}$ - this will be represented by the continuum non-compact $\mathrm{U}(1)$ gauge field $a A_{\mu} /(2 \pi)$, where $a$ is the lattice spacing.

First, we consider quadratic order terms about the saddle point of Eq. (2.8). The most general action has the familiar terms of scalar electrodynamics

$$
\begin{aligned}
\mathcal{S}_{0} & =\int d^{2} r d \tau\left(\sum_{\ell=0}^{q-1}\left[\left|\left(\partial_{\mu}-i A_{\mu}\right) \varphi_{\ell}\right|^{2}+s\left|\varphi_{\ell}\right|^{2}\right]\right. \\
& \left.+\frac{1}{2 e^{2}}\left(\epsilon_{\mu \nu \lambda} \partial_{\nu} A_{\lambda}\right)^{2}\right) .
\end{aligned}
$$

We have rescaled the coupling $e$ here by a factor of $2 \pi$ from Eq. (2.8).

Next, we consider terms which are quartic in the $\varphi_{\ell}$, but which contain no spatial or temporal derivatives. These will be contained in the action $\mathcal{S}_{1}$. We discuss two approaches to obtaining the most general quartic invariants. The first is the most physically transparent, but turns out to be eventually inconvenient for explicit computations. In this approach we use density operators defined in Eq. (1.15), and their simple transformation properties in Eq. (1.16), to build up quartic invariants. In particular, the quartic invariants are only quadratic in the $\rho_{m n}$, and we need only the most general quadratic term invariant under Eq. (1.16). This has the form

$$
\begin{aligned}
\mathcal{S}_{1}=\int d^{2} r d \tau & \left(\sum _ { n = 0 } ^ { q / 2 } \sum _ { m = 0 } ^ { n } \lambda _ { n m } \left[\left|\rho_{n m}\right|^{2}+\left|\rho_{n,-m}\right|^{2}\right.\right. \\
& \left.\left.+\left|\rho_{m n}\right|^{2}+\left|\rho_{m,-n}\right|^{2}\right]\right)
\end{aligned}
$$

However, not all the invariants above are independent, and there are often linear relations between them - this reduces the number of independent coupling constants $\lambda_{n m}$. Determining the linear relations between the couplings turns out to be inconvenient, and we found it easier to proceed by the second method described below.

In the second approach, we first impose only the constraints imposed by the translation operations $T_{x}, T_{y}$. By inspection, it is easy to see that the most general quartic term invariant under these operations has the structure

$$
\mathcal{S}_{1}=\frac{1}{4} \int d^{2} r d \tau \sum_{\ell m n} \gamma_{m n} \varphi_{\ell}^{*} \varphi_{\ell+m}^{*} \varphi_{\ell+n} \varphi_{\ell+m-n}
$$

Here the integers $\ell, m, n, \ldots$ range implicitly from 0 to $q-1$ and all additions over these integers are taken modulo $q$. Imposing in addition the reflection operations in Eq. (2.16), and accounting for the internal symmetries in Eq. (2.21), it is easy to show that the couplings $\gamma_{m n}$ can always be taken to be real and to obey the relations

$$
\begin{aligned}
\gamma_{m n} & =\gamma_{-m,-n} \\
\gamma_{m n} & =\gamma_{m, m-n} \\
\gamma_{m n} & =\gamma_{m-2 n,-n} .
\end{aligned}
$$


We have so far not yet imposed the constraints implied by the lattice rotation $R_{\pi / 2}^{\text {dual }}$. Consequently, Eqs. (2.21) and (2.22) define the most general quartic terms for a system with rectangular symmetry, which may be appropriate in some physical situations. By explicit solution of the constraints defined by Eq. (2.22), we found that the quartic terms are determined by $N_{\text {rect }}$ independent real coupling constants, where

$$
N_{\text {rect }}=\frac{(n+1)(n+2)}{2} \text { for } q=2 n, 2 n+1,
$$

with $n$ a positive integer. Note that the number of couplings grows quite rapidly with increasing $q: N_{\text {rect }} \sim$ $q^{2} / 8$.

Finally, let us consider the consequences of the $R_{\pi / 2}^{\text {dual }}$ symmetry. By applying Eq. (1.13) to Eq. (2.21) we find that $\mathcal{S}_{1}$ remains invariant provided the $\gamma_{m n}$ obey the relations

$$
\gamma_{\bar{m} \bar{n}}=\frac{1}{q} \sum_{m n} \gamma_{m n} \omega^{-[n(\bar{m}-\bar{n})+\bar{n}(m-n)]}
$$

Naïvely, there are $q^{2}$ relations implied by Eq. (2.24), but they are not all independent of each other. By explicitly solving the relations in Eq. (2.24) for a range of $q$ values we found that the number of independent quartic coupling constants for a system with full square lattice symmetry is

$$
N_{\text {square }}=\left\{\begin{array}{cc}
(n+1)^{2} & \text { for } q=4 n, 4 n+1 \\
(n+1)(n+2) & \text { for } q=4 n+2,4 n+3
\end{array}\right.
$$

with $n$ a positive integer. The additional restrictions of square relative to rectangular symmetry reduce the number of independent coupling constants by roughly half at large $q: N_{\text {square }} \sim q^{2} / 16$.

\section{Mean field theory}

This section will examine the mean-field phase diagrams of the general theory $\mathcal{S}_{0}+\mathcal{S}_{1}$ proposed in Section II C. From the discussion in Section I, it is clear that such a procedure can yield a direct second order transition from a superfluid state (with $\left\langle\varphi_{\ell}\right\rangle=0$ ) to an insulating state with density wave order (with $\left\langle\varphi_{\ell}\right\rangle \neq 0$ ). We are interested in determining the possible configurations of values of the $\varphi_{\ell}$ and the associated patterns of density wave order for a range of $q$ values (in this subsection, we will simply write $\left\langle\varphi_{\ell}\right\rangle$ as $\varphi_{\ell}$ because there is no distinction between the two quantities in mean field theory). After determining the $\varphi_{\ell}$, we determined the $\rho_{m n}$ by Eq. (1.15) (using a Lorentzian for the form factor $\left.S(Q)=1 /\left(1+Q^{2}\right)\right)$, and then computed the density wave order using

$$
\delta \rho(\mathbf{r})=\sum_{m, n=-q}^{q-1} \rho_{m n} e^{2 \pi i f\left(m r_{x}+n r_{y}\right)}
$$

We evaluated Eq. (2.26) at $\mathbf{r}$ values corresponding to the sites, bonds, and plaquettes of the direct lattice, and plotted the results in the square lattice figures that appear below. In the formalism of Section II, $\mathbf{r}$ values with integer co-ordinates correspond to the sites of the dual lattice, and hence to plaquettes of the direct lattice. The value of $\delta \rho(\mathbf{r})$ on such plaquette co-ordinates can be considered a measure of the ring-exchange amplitude of bosons around the plaquette. By a similar reasoning, $\mathbf{r}$ values with half-odd-integer co-ordinates represent sites of the direct lattice, and the values of $\delta \rho(\mathbf{r})$ on such sites measure the boson density on these sites. Finally, $\mathbf{r}$ values with $r_{x}$ integer and $r_{y}$ half-odd-integer correspond to horizontal links of the square lattice (and vice versa for vertical links), and the values of $\delta \rho(\mathbf{r})$ on the links is a measure of the mean boson kinetic energy; if the bosons represent a spin system, this is a measure of the spin exchange energy.

Our results appear for a range of $q$ values in the following subsections.

$$
\text { 1. } q=2
$$

After imposing the symmetry relations in Eqs. (2.22) and (2.24), the quartic potential in Eq. (2.21) (defined by $\left.\mathcal{S}_{1}=\int d^{2} r d \tau \mathcal{L}_{4}\right)$ is

$$
\mathcal{L}_{4}=\frac{\gamma_{00}}{4}\left(\left|\varphi_{0}\right|^{2}+\left|\varphi_{1}\right|^{2}\right)^{2}+\frac{\gamma_{01}}{4}\left(\varphi_{0} \varphi_{1}^{*}-\varphi_{0}^{*} \varphi_{1}\right)^{2}
$$

We now make the change of variables

$$
\begin{aligned}
\varphi_{0} & =\frac{\zeta_{0}+\zeta_{1}}{\sqrt{2}} \\
\varphi_{1} & =-i \frac{\zeta_{0}-\zeta_{1}}{\sqrt{2}} .
\end{aligned}
$$

As we will discuss in detail in Section III A, the new variables have the advantage of realizing a permutative representation of the PSG, i.e. all representation matrices of the PSG have only one non-zero unimodular element in every row and column. From Eqs. (1.12), (1.13), and $(2.28)$ it is easy to deduce that in the $\zeta_{\ell}$ variables,

$$
\begin{aligned}
T_{x}=\left(\begin{array}{cc}
0 & -i \\
i & 0
\end{array}\right) \quad & ; T_{y}=\left(\begin{array}{ll}
0 & 1 \\
1 & 0
\end{array}\right) \\
R_{\pi / 2}^{\text {dual }} & =\left(\begin{array}{cc}
0 & e^{-i \pi / 4} \\
e^{i \pi / 4} & 0
\end{array}\right) .
\end{aligned}
$$

The action in Eq. (2.27) reduces to

$$
\mathcal{L}_{4}=\frac{\gamma_{00}}{4}\left(\left|\zeta_{0}\right|^{2}+\left|\zeta_{1}\right|^{2}\right)^{2}-\frac{\gamma_{01}}{4}\left(\left|\zeta_{0}\right|^{2}-\left|\zeta_{1}\right|^{2}\right)^{2}
$$

The result in Eq. (2.30) is identical to that found in earlier studies ${ }^{13,14}$ of the $q=2$ case.

Minimizing the action implied by Eq. (2.30), it is evident that for $\gamma_{01}<0$ there is a one parameter family of 


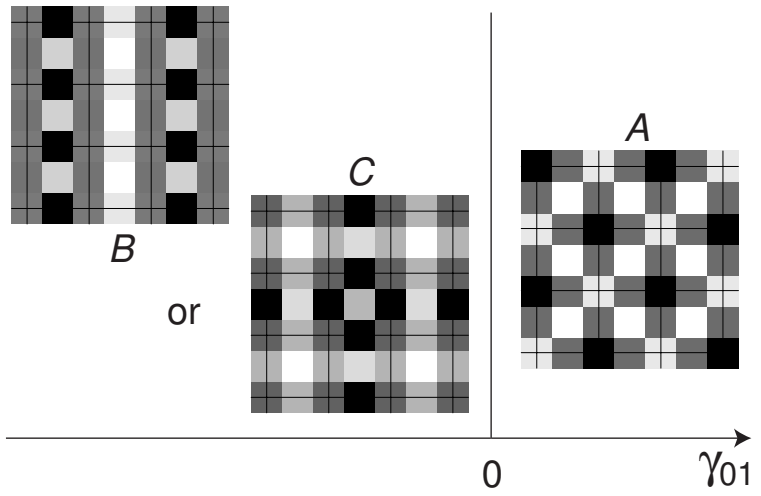

FIG. 3: Mean field phase diagram of $\mathcal{S}_{0}+\mathcal{S}_{1}$ defined in Eqs. (2.19) and (2.21). The couplings obey Eqs. (2.22) and (2.24). We assumed $s=-1$ to obtain an insulating state, and $\gamma_{00}=1$. Plotted are the values of $\delta \rho(\mathbf{r})$, defined as in Eq. (2.26), on the sites, links and plaquettes of the direct lattice: the lines represent links of the direct lattice. As discussed in the text, these values represent the boson density, kinetic energy, and ring-exchange amplitudes respectively. The saddle point values of the fields in the states are shown in Eq. (2.31). The choice between the states $B$ and $C$ is made by a eighth order term in the action.

gauge-invariant solutions in which the relative phase of $\zeta_{0}$ and $\zeta_{1}$ remains undetermined. Computing the values of $\delta \rho(\mathbf{r})$ establishes that this phase is physically significant, because it leads to distinguishable density wave modulations. This indicates that the phase will be determined by higher order terms in the effective potential. As shown in earlier work ${ }^{13}$, one needs to go to a term which is eighth order in the $\varphi_{\ell}$ before the value of $\arg \left(\zeta_{0} / \zeta_{1}\right)$ is pinned at specific values. In the present formalism, the required eighth order term is $\rho_{0,1}^{2} \rho_{1,0}^{2}$, which is invariant under all the transformations in Eq. (1.16); this contains a term $\sim\left(\zeta_{0} \zeta_{1}^{*}\right)^{4}+$ c.c.

Our specific results from the mean field theory for $q=2$ are summarized in Fig 3. The state (A) has an ordinary charge density wave (CDW) at wavevector $(\pi, \pi)$. The other two states are VBS states in which all the sites of the direct lattice remain equivalent, and the VBS order appears in the (B) columnar dimer or (C) plaquette pattern. The saddle point values of the fields associated with these states are:

$$
\begin{aligned}
& (A): \zeta_{0} \neq 0, \zeta_{1}=0 \text { or } \zeta_{0}=0, \zeta_{1} \neq 0 . \\
& (B): \zeta_{0}=e^{i n \pi / 2} \zeta_{1} \neq 0 . \\
& (C): \quad \zeta_{0}=e^{i(n+1 / 2) \pi / 2} \zeta_{1} \neq 0,
\end{aligned}
$$

where $n$ is any integer. At quartic order, the states $B$ and $C$ are degenerate with all states with $\zeta_{0}=e^{i \theta} \varphi_{1}$, with $\theta$ arbitrary; the value of $\theta$ is selected only by the eighth order term.

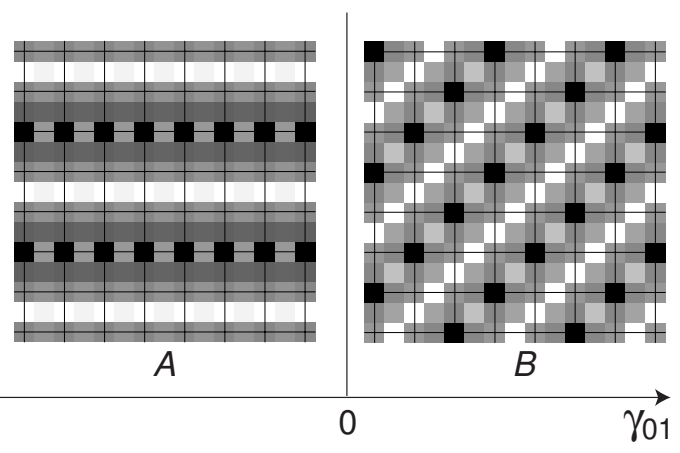

FIG. 4: As in Fig 3 but for $q=3$

$$
\text { 2. } q=3
$$

Now there are $3 \varphi_{\ell}$ fields, and the quartic potential in Eq. (2.27) is replaced by

$$
\begin{aligned}
\mathcal{L}_{4} & =\frac{\gamma_{00}}{4}\left(\left|\varphi_{0}\right|^{2}+\left|\varphi_{1}\right|^{2}+\left|\varphi_{2}\right|^{2}\right)^{2} \\
& +\frac{\gamma_{01}}{2}\left(\varphi_{0}^{*} \varphi_{1}^{*} \varphi_{2}^{2}+\varphi_{1}^{*} \varphi_{2}^{*} \varphi_{0}^{2}+\varphi_{2}^{*} \varphi_{0}^{*} \varphi_{1}^{2}+\right.\text { c.c. } \\
& \left.-2\left|\varphi_{0}\right|^{2}\left|\varphi_{1}\right|^{2}-2\left|\varphi_{1}\right|^{2}\left|\varphi_{2}\right|^{2}-2\left|\varphi_{2}\right|^{2}\left|\varphi_{0}\right|^{2}\right)(2.32)
\end{aligned}
$$

We show in Appendix E that there is now no transformation of the $\varphi_{\ell}$ variables which realize a permutative representation of the PSG, and in which Eq. (2.32) may take a more transparent form. The results of the minimization of $\mathcal{S}_{0}+\mathcal{S}_{1}$ for the insulating phases are shown in Fig 4. The states have stripe order, one along the diagonals, and the other along the principle axes of the square lattice. Both states are 6-fold degenerate, and the characteristic saddle point values of the fields are

$$
\begin{gathered}
(A): \varphi_{0} \neq 0, \varphi_{1}=\varphi_{2}=0 \text { or } \\
e^{i 4 n \pi / 3} \varphi_{2}=e^{i 2 n \pi / 3} \varphi_{1}=\varphi_{0} \neq 0 \\
(B): \varphi_{0}=\varphi_{1}=e^{ \pm 2 i \pi / 3} \varphi_{2} \\
\text { and permutations. }
\end{gathered}
$$

$$
\text { 3. } q=4
$$

For $q=4$, there are 4 independent quartic invariants in Eq. (2.21), and they can be combined into the following forms.

$$
\begin{aligned}
& I_{1}=\rho_{0,0}^{2}=\left(\left|\varphi_{0}\right|^{2}+\left|\varphi_{1}\right|^{2}+\left|\varphi_{2}\right|^{2}+\left|\varphi_{3}\right|^{2}\right)^{2} \\
& I_{2}=\rho_{2,2}^{2}=\left(\varphi_{0}^{*} \varphi_{2}+\varphi_{2}^{*} \varphi_{0}-\varphi_{1}^{*} \varphi_{3}-\varphi_{3}^{*} \varphi_{1}\right)^{2} \\
& I_{3}=\left|\varphi_{0}\right|^{2}\left|\varphi_{1}\right|^{2}+\left|\varphi_{1}\right|^{2}\left|\varphi_{2}\right|^{2}+\left|\varphi_{2}\right|^{2}\left|\varphi_{3}\right|^{2}+\left|\varphi_{3}\right|^{2}\left|\varphi_{0}\right|^{2} \\
& -\varphi_{0}^{*} \varphi_{1}^{*} \varphi_{2} \varphi_{3}-\varphi_{1}^{*} \varphi_{2}^{*} \varphi_{3} \varphi_{0}-\varphi_{2}^{*} \varphi_{3}^{*} \varphi_{0} \varphi_{1}-\varphi_{3}^{*} \varphi_{0}^{*} \varphi_{1} \varphi_{2} \\
& I_{4}=\varphi_{0}^{*} \varphi_{2}^{*}\left(\varphi_{1}^{2}+\varphi_{3}^{2}\right)+\text { c.c. }+\varphi_{1}^{*} \varphi_{3}^{*}\left(\varphi_{0}^{2}+\varphi_{2}^{2}\right)+\text { c.c. } \\
& \quad-4\left|\varphi_{0}\right|^{2}\left|\varphi_{2}\right|^{2}-4\left|\varphi_{1}\right|^{2}\left|\varphi_{3}\right|^{2}
\end{aligned}
$$

Now as for $q=2$, the invariants for $q=4$ do simplify by redefinitions of the fields analogous to Eq. (2.28), which 
realize a permutative representation of the PSG. We define

$$
\begin{aligned}
\varphi_{0} & =\left(\zeta_{0}+\zeta_{1}\right) / \sqrt{2} \\
\varphi_{2} & =\left(\zeta_{0}-\zeta_{1}\right) / \sqrt{2} \\
\varphi_{1} & =\left(\zeta_{3}+\zeta_{2}\right) / \sqrt{2} \\
\varphi_{3} & =\left(\zeta_{3}-\zeta_{2}\right) / \sqrt{2}
\end{aligned}
$$

The PSG operations on the $\zeta_{\ell}$ can then easily be shown to take the permutative form specified in Eq. (C5) (in a slightly different notation). In the new variables, the quartic potential can be written in the following form

$$
\begin{aligned}
\mathcal{L}_{4} & =\frac{u}{2}\left(\left|\zeta_{0}\right|^{4}+\left|\zeta_{1}\right|^{4}+\left|\zeta_{2}\right|^{4}+\left|\zeta_{3}\right|^{4}\right) \\
& +v_{1}\left(\left|\zeta_{0}\right|^{2}+\left|\zeta_{2}\right|^{2}\right)\left(\left|\zeta_{1}\right|^{2}+\left|\zeta_{3}\right|^{2}\right) \\
& +v_{2}\left(\left|\zeta_{0}\right|^{2}\left|\zeta_{2}\right|^{2}+\left|\zeta_{1}\right|^{2}\left|\zeta_{3}\right|^{2}\right) \\
& -\frac{\lambda}{2}\left(\zeta_{0}^{* 2} \zeta_{1}^{2}+\zeta_{1}^{* 2} \zeta_{0}^{2}-\zeta_{1}^{* 2} \zeta_{2}^{2}-\zeta_{2}^{* 2} \zeta_{1}^{2}\right. \\
& \left.+\zeta_{2}^{* 2} \zeta_{3}^{2}+\zeta_{3}^{* 2} \zeta_{2}^{2}+\zeta_{3}^{* 2} \zeta_{0}^{2}+\zeta_{0}^{* 2} \zeta_{3}^{2}\right)
\end{aligned}
$$

where $u, v_{1,2}$ and $\lambda$ are coupling constants. Notice that at $\lambda=0$ the action is independent of the relative phases of the $\zeta_{\ell}$, and so there is a 3 parameter family of degenerate states. It is therefore convenient to first determine the minima at $\lambda=0$ (which can be done analytically), and to then determine the fate of the minima so found at small $\lambda$. The results of such a procedure are summarized in Fig 5 . The states in Fig 5 are characterized by the following parameter values

$(A$ and $B)$ : One of $\zeta_{0}$ or $\zeta_{2}$ non-zero, one of $\zeta_{1}$ or $\zeta_{3}$ non-zero, magnitudes of non-zero $\zeta_{\ell}$ equal

$C$ : Only one of $\left|\zeta_{\ell}\right|$ non-zero

$D:$ Either $\zeta_{0}=\zeta_{2}=0$ and $\left|\zeta_{1}\right|=\left|\zeta_{3}\right| \neq 0$, or $\zeta_{1}=\zeta_{3}=0$ and $\left|\zeta_{0}\right|=\left|\zeta_{2}\right| \neq 0$

$E$ : All $\left|\zeta_{\ell}\right|$ equal and non-zero

By inserting the saddle point values of state $D$ in Eq. (2.36) we observe that this state has a continuous degeneracy with $\zeta_{1}=e^{i \theta} \zeta_{3}$ (for the first choice of the saddle point) and $\theta$ arbitrary. We expect that higher order terms in the action will select the value of $\theta$, and we show the higher symmetry states at special values of $\theta$ in Fig 6.

The phases in Fig 5 all survive for a finite range of $\lambda$. However, for large enough $|\lambda|$ a number of additional phases, with distinct patterns of lattice symmetry breaking, appear. We have not determined the full phase diagram in the three-dimensional $v_{1}, v_{2}, \lambda$ space, but here merely list the additional phases that were found in our numerical analysis. The new large $|\lambda|$ phases are shown in Fig 7, and the typical saddle points had the following characteristics:

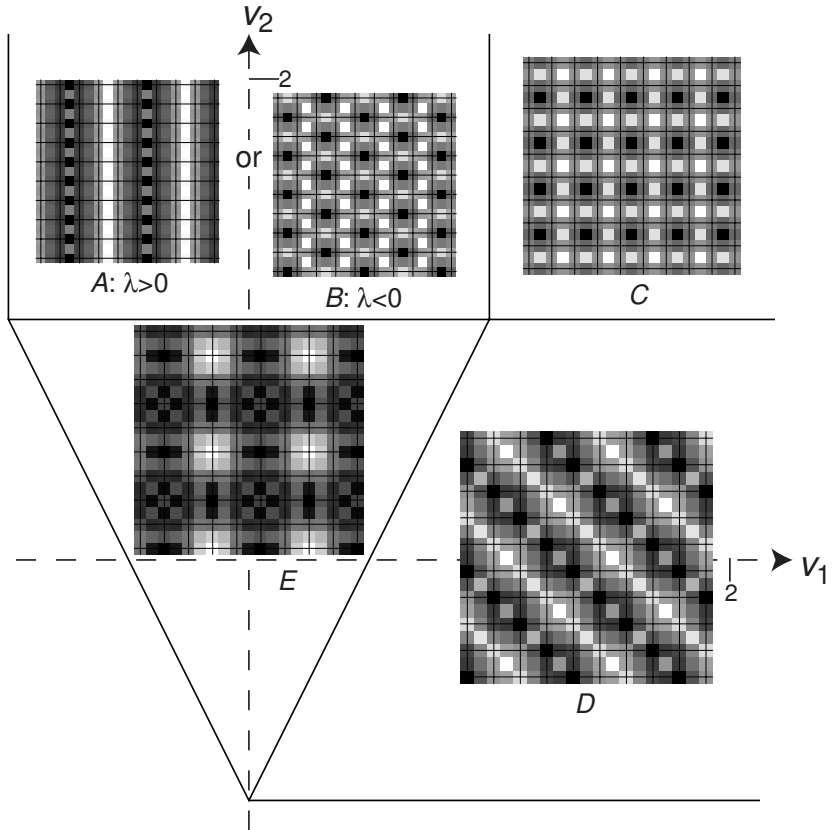

FIG. 5: As in Fig 3, but for $q=4$ with the quartic potential as in Eq. (2.36). We have set $u=1$, and then determined the phase diagram as function of $v_{1}$ and $v_{2}$ for infinitesimal values of $\lambda$. The dashed lines represent the $v_{1}$ and $v_{2}$ axes, while the full lines are phase boundaries.

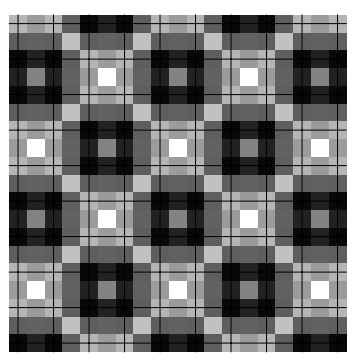

$D_{1}$

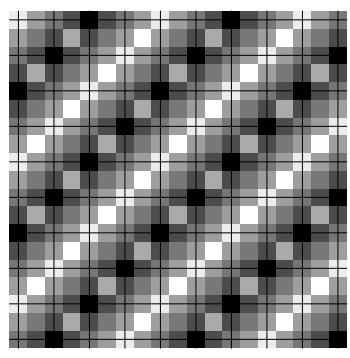

$D_{2}$
FIG. 6: Higher symmetry cases of the state $D$ in Fig. 5. With the angle $\theta$ defined as in the text, state $D_{1}$ corresponds to $\theta=n \pi / 2$, and the state $D_{2}$ to $\theta=(n+1 / 2) \pi / 2$ ( $n$ integer $)$.

$$
\begin{aligned}
F & : \zeta_{0}=\zeta_{3}, \zeta_{1}=\zeta_{2},\left|\zeta_{0}\right| \neq\left|\zeta_{1}\right| \\
G \quad: & \zeta_{0}=i \zeta_{2},\left|\zeta_{1}\right| \neq\left|\zeta_{0}\right|, \arg \left(\zeta_{0}\right)=\arg \left(\zeta_{1}\right)+\pi \\
& \quad \text { and } \zeta_{3}=0 \\
H \quad: & \zeta_{3}=i \zeta_{0}, \zeta_{1}=i \zeta_{2},\left|\zeta_{0}\right| \neq\left|\zeta_{1}\right| \\
I & : \quad \zeta_{1}=i \zeta_{3},\left|\zeta_{1}\right| \neq\left|\zeta_{2}\right|, \arg \left(\zeta_{2}\right)=\arg \left(\zeta_{1}\right) \\
& \quad \text { and } \zeta_{0}=0 .
\end{aligned}
$$

\section{General $q$}

The insulating states obtained above clearly have the dimensions of their unit cells constrained by the value of $q$, and we will discuss these constraints more explicitly 


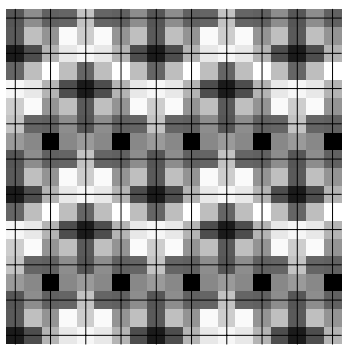

F

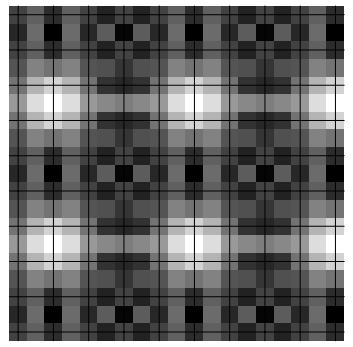

H

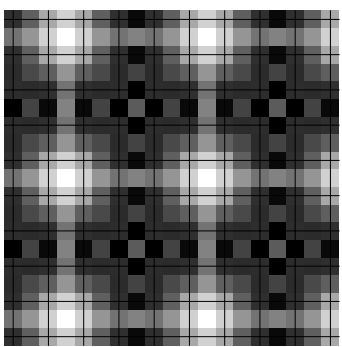

G

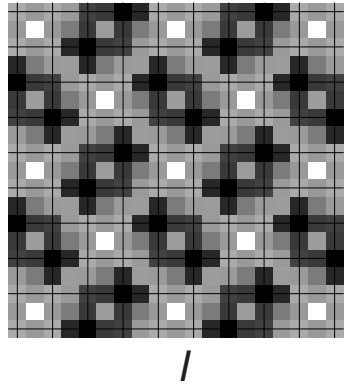

FIG. 7: Additional insulating mean-field states for $q=4$ with the quartic potential as in Eq. (2.36). These states appear for larger values of $|\lambda|$ and the field configurations are described in the text.

here with special attention to the value $q=16$. From Eq. (1.6) and the analysis of II, this value of $q$ is of particular relevance to the cuprates at the hole density of $1 / 8$. From Eq. (2.25), we note that the $q=16$ case has 25 independent quartic coupling constants. The full parameter space of insulating states is therefore of immense complexity, and we will not attempt to map out any phase diagram. Instead we shall examine the spatial structure of a few simple configurations of the $\varphi_{\ell}$.

Let us assume that the insulating state has a unit cell of size $a \times b$ lattice sites ( $a, b$ integers). We now discuss the general constraints on the values of $a$ and $b$. From the allowed values of the wavevectors in Eq. (1.14) we see that generically we will have $a=q$ and $b=q$. However, it could be that the $\varphi_{\ell}$ take special values so that some of the $\rho_{m n}$ vanish. In this case, the periods $a$ and $b$ could be any integer divisor of $q$. However, an interesting property of Eq. (1.15) is that it is not possible to simultaneously reduce the values of both $a$ and $b$. The non-commutativity of $T_{x}$ and $T_{y}$ in Eq. (1.8) effectively imposes an "uncertainty relation" between their values so that the product $a b$ must be a multiple of $q$ : intuitively, we see this as a requirement that the unit cell must contain an integer number of bosons. Summarizing, the values of $a$ and $b$ are expected to be constrained by the requirements that

$$
\frac{q}{a}, \frac{q}{b}, \frac{a b}{q} \text { are all integers. }
$$

To illustrate the origin of the constraints in Eq. (2.39), let us consider the $q=16$ case, and attempt to construct insulating states with $a$ and $b$ as small as possible. Motivated by the application to the cuprates at hole density $\delta=1 / 8$, let us look for solutions in which $b$ is 4 or smaller. From Eq. (1.15) it is clear that a simple way to achieve this is to take $\varphi_{\ell}=0$ unless $\ell$ is a multiple of 4 , so that $\rho_{m n}$ is zero unless $n$ is a multiple of 4 . A generic solution with this property has $\rho_{m n}$ non-zero for all $m$, and hence a period $a=16$. To obtain a smaller value of $a$ we must make the $\rho_{m n}$ vanish for as large a set of $m$ values as possible. However, with this particular choice of the $\varphi_{\ell}$ notice that $\rho_{m n}$ is proportional to $\rho_{m+4, n}$. So the best we can do is to have $\rho_{m n}$ non-zero only for $m$ a multiple of 4 . In this case we obtain $a=4$, which results in a unit cell consistent with Eq. (2.39).

We used the above strategy to identify a number of $\varphi_{\ell}$ configurations with highly symmetric, small unit cell density modulations. A few sample results are shown in Fig 8. All unit cells are clearly consistent with Eq. (2.39).

\section{E. Renormalization group analysis}

We now briefly address fluctuation effects across the mean field transitions found in Section IID. The analysis follows previous work ${ }^{38,39}$ on scalar quantum electrodynamics in three dimensions who examined the theory $\mathcal{S}_{0}+\mathcal{S}_{1}$ for the case where the quartic interactions had a full $\mathrm{U}(q)$ invariance. Here we will extend the earlier one loop results to the particular quartic couplings in Eq. (2.21). Unfortunately, we find runaway flows to strong coupling for all values of $q$ we have studied, and no stable fixed points which are accessible in a one loop analysis.

For the renormalization group (RG) analysis it is useful to write the quartic terms in $\mathcal{S}_{1}$ in the more general form

$$
\mathcal{S}_{1}=\frac{1}{4} \int d^{2} r d \tau \sum_{\ell m n i} u_{\ell m ; n i} \varphi_{\ell}^{*} \varphi_{m}^{*} \varphi_{n} \varphi_{i}
$$

where

$$
u_{\ell m ; n i} \equiv \delta_{\ell+m, n+i} \gamma_{m-\ell, n-\ell} .
$$

These couplings obey the symmetry relations

$$
\begin{aligned}
& u_{\ell m ; n i}=u_{m \ell ; n i} \\
& u_{\ell m ; n i}=u_{\ell m ; i n} \\
& u_{\ell m ; n i}=u_{n i ; \ell m} \\
& u_{\ell m ; n i}=u_{\ell+j, m+j ; n+j, i+j},
\end{aligned}
$$

(as always, all index arithmetic is modulo $q$ ) and the trace identity ${ }^{40}$

$$
\sum_{\ell} u_{\ell m ; \ell i}=U \delta_{m i}
$$

with $U \equiv \sum_{\ell} \gamma_{\ell 0}$. 


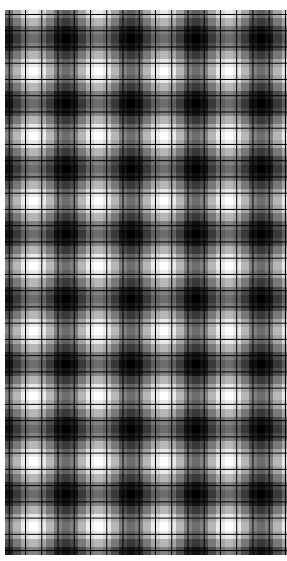

(a)

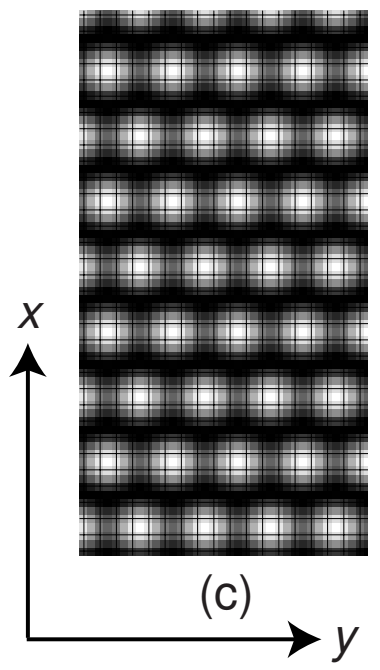

FIG. 8: A few $\varphi_{\ell}$ configurations for $q=16$ with higher symmetry and unit cells smaller than the maximal $16 \times 16$. All figures have $\varphi_{\ell}=0$ unless $\ell$ is a multiple of 4 . (a) $\varphi_{0}=1$, $\varphi_{4}=1, \varphi_{8}=1, \varphi_{12}=1,4 \times 4$ unit cell. (b) $\varphi_{0}=1, \varphi_{4}=-1$, $\varphi_{8}=1, \varphi_{12}=1,16 \times 4$ unit cell. (c) $\varphi_{0}=1, \varphi_{4}=1$, $\varphi_{8}=-1, \varphi_{12}=-1,8 \times 4$ unit cell. (d) $\varphi_{0}=1, \varphi_{4}=0$, $\varphi_{8}=1, \varphi_{12}=0,8 \times 2$ unit cell.

The RG equations can be derived by a simple generalization of the methods in Refs. 39,40. At one loop order the results are

$$
\begin{aligned}
\frac{d e^{2}}{d l} & =\epsilon e^{2}-\frac{C q}{3} e^{4} \\
\frac{d u_{\ell m ; n k}}{d l} & =(\epsilon-2 \eta) u_{\ell m ; n k}-C \sum_{i j}\left(\frac{1}{2} u_{\ell m ; i j} u_{i j ; n k}\right. \\
& \left.+u_{\ell i ; n j} u_{m j ; k i}+u_{\ell i ; k j} u_{m j ; n i}\right) \\
& -a_{2} e^{4} C\left(\delta_{\ell n} \delta_{m k}+\delta_{\ell k} \delta_{m n}\right) \\
\eta & =-a_{1} e^{2} C .
\end{aligned}
$$

The equations have been derived in $3-\epsilon$ spatial dimen- sions and $\eta$ is the anomalous dimension of the $\varphi_{\ell}$ field. The numerical constants $C, a_{1,2}$ can be obtained both in the $\epsilon$ expansion and in the fixed dimension RG performed directly in two spatial dimensions. For the $\epsilon$ expansion we have $C^{-1}=8 \pi^{2}, a_{1}=3, a_{2}=6$. For the fixed dimension expansion we have $C^{-1}=8 \pi, a_{1}=8 / 3, a_{2}=4$. Using Eq. (2.41), the equation for the quartic couplings becomes

$$
\begin{aligned}
\frac{d \gamma_{m n}}{d l} & =(\epsilon-2 \eta) \gamma_{m n}-C_{d} \sum_{\ell}\left(\frac{1}{2} \gamma_{m \ell} \gamma_{2 n-m, n-\ell}\right. \\
& \left.+\gamma_{\ell n} \gamma_{m+n-\ell, n}+\gamma_{\ell, m-n} \gamma_{\ell-n, m-n}\right) \\
& -a_{2} e^{4} C_{d}\left(\delta_{n 0}+\delta_{m n}\right)
\end{aligned}
$$

We have verified that this flow equation for the couplings $\gamma_{m n}$ preserves the symmetry constraints in Eq. (2.22) (for rectangular symmetry) and Eq. (2.24) (for square symmetry).

We searched for fixed points of Eqs. (2.44) and Eqs. (2.45) for small values of $q$, and found that all were unstable (as expected from Refs. 38,39). The analysis becomes rapidly more complicated with increasing values of $q$, and we do not have general understanding of the flow structure of these equations.

\section{F. Deviations from density $p / q$}

Our formalism has so far been explicitly designed for rational boson densities $f$ obeying Eq. (1.11). In principle, it would appear then we can model essentially any density simply by taking $q$ large enough. However, this is not an attractive way to proceed for reasons discussed below.

First, recall that there are $q$ minima in the Brillouin zone, and so these minima become more closely spaced with increasing $q$. So, to consider these minima independent degrees of freedom (as is implicitly done in our continuum field theory), we can only work at momenta which are significantly smaller than the spacing between the minima. Hence the continuum theory of Section II C only applies beyond a large length scale which increases linearly with $q$.

Second, the $q$ minima are very shallow for large $q$. In other words, the bandwidth of the lowest lowest energy Hofstadter band containing these minima is very narrow. When integrating out the vortex modes away from the minima to arrive at the continuum field theory, one encounters energy denominators determined by this bandwidth. In fact, this is likely the most severe restriction on the naïve application of the $q$-vortex method. In particular, one may argue that for $p=1$ (and likely for any $p$ by some refinement of this argument) the bandwidth of the lowest Hofstadter band is exponentially small, $O\left(e^{-c q}\right)$ with some $O(1)$ constant $c$, for large $q .{ }^{65}$ This is a much smaller energy scale than the splitting between Landau 
bands, which is also small but $O(1 / q)$ for large $q$. A quantitative physical consequence of the narrow bandwidth is a reduction of the vortices' tendency to condense, i.e. an enhancement of the domain of the boson superfluid state.

As a consequence of these difficulties, it is preferable to take the continuum limit appropriate to the low energy fluctuations of some nearby insulating commensurate state with a moderate value of $q$. The actual density of the boson system may not exactly equal $p / q$. However, it is easy to modify the theory for the system at density $p / q$ (presented in Section II C), to allow for this density deviation. As the boson density is the average $A_{\mu}$ 'magnetic' flux, we simply have to replace the action $\mathcal{S}_{0}$ in Eq. (2.19) by

$$
\begin{aligned}
\mathcal{S}_{0} & =\int d^{2} r d \tau\left(\sum_{\ell=0}^{q-1}\left[\left|\left(\partial_{\mu}-i A_{\mu}\right) \varphi_{\ell}\right|^{2}+s\left|\varphi_{\ell}\right|^{2}\right]\right. \\
& \left.+\frac{1}{2 e^{2}}\left(\epsilon_{\mu \nu \lambda} \partial_{\nu} A_{\lambda}-\frac{2 \pi \delta f}{a^{2}} \delta_{\mu \tau}\right)^{2}\right)
\end{aligned}
$$

where $\delta f$ is the difference between $p / q$ and the actual boson density, and $a$ is the lattice spacing. All the higher order terms in the action remain as discussed in Section II C.

The theory in Eq. (2.46) has a structure similar to the Ginzburg-Landau model for a 'superconductor' in a 'magnetic' field, and has corresponding possibilities for its phases. It can behave like a type I 'superconductor' and expel the flux $\delta f$, while condensing the $\varphi_{\ell}$ : this clearly yields the commensurate Mott insulator with density $p / q$. In the 'normal' phase of this 'superconductor' the $\varphi_{\ell}$ are uncondensed and flux $\delta f$ pierces the system: this is the superfluid with density $p / q+\delta f$. This 'normal' phase can also have a condensate of vortex-anti-vortex composites with some of $\left\langle\rho_{m n}\right\rangle$ non-zero, and this is a supersolid with density $p / q+\delta f$, but with density wave order similar to that in the commensurate Mott insulator. Finally, the model Eq. (2.46) can also behave like a type II 'superconductor' and allow the 'flux' to penetrate the system in an Abrikosov lattice: this is an incommensurate insulator (or floating Wigner solid) in which the particle density is $p / q+\delta f$. Partial 'flux' penetration is also possible, and this yields Mott insulators at other densities.

\section{BOSON FRACTIONALIZATION}

The discussion of the previous sections generalizes the dual effective action of Refs. 15 (and its predecessors) for $f=1 / 2$. In that work, it was pointed out that for $f=1 / 2$ the theory with $\gamma_{01}<0$ is itself equivalent to a fractionalized representation in which the elementary boson is split into two "half" bosons, carrying equal ( $\frac{1}{2}$ the elementary value) physical charge but opposite values of an emergent $U(1)$ gauge charge. These fractional particles are deconfined at the critical point in the sense that the gauge magnetic flux is conserved by the fixed point theory. In the language of the present paper, this conservation law is nothing but the conservation of the difference of the two $\left(\zeta_{0}\right.$ and $\left.\zeta_{1}\right)$ vorticities.

Two important features are critical to this conclusion. First, we used the equivalence of the dual 2 -vortex action - in a particular regime $\left(\gamma_{01}<0\right)$ - to a fractionalized 1/2-boson representation. This mapping is nontrivial and indeed does not generalize to all $f$, as we will discuss below. Second, even when such a representation does obtain, deconfinement at the QCP is contingent upon the fixed point theory possessing the appropriate emergent conservation law(s). This is true for $f=1 / 2$ roughly because the most relevant operator to violate the conservation law $\left(\left(\zeta_{0}^{*} \zeta_{1}\right)^{4}+\right.$ h.c. $)$ is sufficiently high order that it can be persuasively argued ${ }^{15}$ to be irrelevant. In general, the relevance or irrelevance of such operators is far from obvious, as the interacting $q$-vortex gauge theory in $2+1$ dimensions is strongly coupled, so that the critical indices needed to answer this question are not calculable analytically. For this reason, we will have little to say in this paper about this second issue, which is best resolved by numerical (Monte Carlo) studies.

In this section we will however discuss the first requirement for a fractionalized boson interpretation of these Mott QCPs. Perhaps surprisingly, we will see that this can be satisfied in a theory with full square lattice symmetry only for a rather special set of values of rational $f$. We do not at present have a general criteria to determine these values of $f$. By explicit example, we find that a fractionalized formulation is possible for $f=1 / 2,1 / 4,1 / 8,1 / 9$, and have proven (see below and Appendix E) that none exists for $f=1 / 3$. We speculate that the sequences of $f=p / q$ with $q=n^{2}, 2 n^{2}$ and integer $n$ all admit a fractionalized formulation. If the microscopic symmetry is relaxed from square to rectangular (i.e. invariance under only twofold rather than fourfold rotations), however, the fractionalization requirements can be satisfied for any rational $f$. We show how these conclusions can be obtained both from an analysis of the dual $q$-vortex action of this paper, and from a complementary direct analysis of the original boson problem. The agreement between these two completely distinct methods provides an important check on the results, as well as useful physical interpretation. The direct method is a generalization of the $U(1)$ gauge theory approach employed in Ref. 15.

Although this analysis provides a formal means of understanding fractionalization at such Mott QCPs, a more physical picture is also desirable. In Ref. 45, Levin and Senthil provided such a simple real-space picture of charge- $1 / 2$ excitations for $f=1 / 2$ coming from the Mott side of the transition. They showed that these particles can be understood as $Z_{4}$ vortices - points of connection of four elementary domain walls - in the density wave (valence bond solid) order parameter. For the specific case of $f=1 / 4$, we discuss, in Section III C, a similar picture - as $Z_{4} \times Z_{4}$ vortices - for the charge $1 / 4$ excitations that 
(may) become deconfined at the Mott transition.

\section{A. Fractionalization in the vortex picture}

Let us consider the general $q$-vortex action at filling $f=p / q$. By blind analogy with half filling $(f=1 / 2)$, one might expect this to describe a QCP in which the elementary boson has been fractionalized into $q$ bosons with charge $1 / q$. If we examine this proposition in a little detail, it becomes clear that this is not quite generally correct.

For half-filling, ${ }^{15}$ this conclusion was based on a duality mapping of the 2-vortex theory back to a half-boson theory. The physical content of this mapping is that a 'vortex' in a single vortex field $\varphi_{\ell}$ (this is a sometimes confusing double duality which unfortunately cannot be avoided in this particular method of analysis) is a point particle whose creation or annihilation operator becomes the fundamental field in a new representation. Here and in the following we use single quotes to distinguish this 'vortex' from a true physical vortex, which is created by a linear combination of $\varphi_{\ell}$ fields. As in any other GinzburgLandau-like theory, such a 'vortex' will bind a quantized amount of 'flux' $\int d^{2} r \epsilon_{i j} \partial_{i} A_{j}$. The 'flux' however is $2 \pi$ times the physical charge (boson number), so this 'vortex' is actually a particle carrying some amount of boson charge, which we will see is fractional under favorable circumstances.

The above argument has one crucial un-stated assumption: that vortex-like topological defects in the $\varphi_{\ell}$ fields are well-defined. This is true only if the magnitudes $\left|\varphi_{\ell}\right|$ are constrained to be approximately constant, i.e. that the dominant terms in the action are minimized by this condition. Moreover, phases of these fields should be unconstrained. Formally, this requirement is necessary to pass from the soft-spin Ginzburg-Landau theory of the $\varphi_{\ell}$ fields to a hard-spin description in terms of phaseonly variables. Duality transformations are formulated in a hard-spin description.

Actually, we can relax this requirement slightly, as already hinted by the mean-field analysis of the previous section. In particular, since the quadratic action $\mathcal{S}_{0}$ is invariant under a global $U(q)$ unitary rotation of the $\varphi_{\ell}$ fields, it is sufficient that some unitarily transformed fields $\zeta_{\ell}=\sum_{\ell^{\prime}} U_{\ell \ell^{\prime}} \varphi_{\ell^{\prime}}$ (with $U^{\dagger} U=1$ ) can be regarded as having approximately constant magnitude, $\left|\zeta_{\ell}\right|=\bar{\varphi}$, a constant, but arbitrary phases. One may think of this as follows. Consider the evolution of the system from the superconducting to insulating state, by reducing $s$ starting from $s>0$. Due to fluctuations, the actual location of the critical point $s=s_{c}$ at which vortex condensation occurs is reduced to $s_{c}<0$. Thus one may expect that locally, for $s_{c} \lesssim s<0$, the $\zeta_{\ell}$ fields may develop some non-zero magnitude. This occurs because of a balance between the negative quadratic term $-|s| \sum_{\ell}\left|\zeta_{\ell}\right|^{2}$ and the quartic terms in $\mathcal{L}_{4}$. From the form of the negative quadratic term, this balance clearly favors states with a non-zero generalized "radius" $\sqrt{\sum_{\ell}\left|\zeta_{\ell}\right|^{2}}$ in $\zeta_{\ell}$ space. The preferred directions in this $2 q$-dimensional (due to real and imaginary parts of each $\zeta_{\ell}$ ) space, however, are determined by the terms in $\mathcal{L}_{4}$. To obtain a phase-only description, one needs that the dominant terms in $\mathcal{L}_{4}$ favor states with $\left|\zeta_{\ell}\right|=\bar{\varphi}$.

This requirement places strong constraints on the form of $\mathcal{L}_{4}$. In particular, one must have that, for fixed $\sum_{\ell}\left|\zeta_{\ell}\right|^{2}=q \bar{\varphi}^{2}$, the minima of $\mathcal{L}_{4}$ satisfy $\left|\zeta_{\ell}\right|=\bar{\varphi}$ for all $\ell$. Explicit examples with rotational invariance can be seen for $q=2,4$ from Sec. II D 1,IID 3. For $q=2$, this is true if $\gamma_{01}<0$. For $q=4$ this is true in region $\mathrm{E}$ when $\lambda=0$. Thus if $\lambda$ is irrelevant in region $\mathrm{E}$ at the QCP, the requirement is satisfied. On the other hand, for $q=3$, it is clearly not satisfied. For $\gamma_{01}=0$, there is a full $2 q-1=5$-sphere of degenerate states, clearly of different topology than the 3-torus of states of three angular variables. For $\gamma_{01}>0$, the degeneracy of minima of $\mathcal{L}_{4}$ is just a discrete multiple of the overall $U(1)$ (singular angular degree of freedom) gauge symmetry.

We do not have a general analysis of when the constant amplitude condition can be satisfied. One can, however, place a necessary condition on the existence of any Lagrangian invariant under the symmetries of the problem for which all minimum action configurations satisfy $\left|\zeta_{\ell}\right|=\bar{\varphi}$ and conversely all such configurations have minimal action. In particular, for any such configuration, any new configuration equivalent to it by symmetry must have equal action. Thus if these are the unique configurations with minimal action, all symmetry operations must preserve this condition. That is, the space of states with $\left|\zeta_{\ell}\right|=\bar{\varphi}$ should be closed under all symmetry operations. Unfortunately, this is not trivial to check, since we still have a large freedom to choose the unitary transformation $U_{\ell \ell^{\prime}}$ defining the $\zeta_{\ell}$ fields.

For the simpler case in which the requirement of rotational invariance is removed, i.e. for a system with rectangular rather than square symmetry, it is straightforward to see that this condition can be achieved for all $f$. This is easily seen from Eqs. (2.21) (2.22). In particular, a quartic term of the form $\mathcal{L}_{4}=\frac{u}{2} \sum_{\ell}\left|\varphi_{\ell}\right|^{4}$ is allowed for all $q$, and favors equal amplitude states. It is clearly invariant under all symmetry operations save rotations. Hence no unitary rotation is required, and we can simply take $\zeta_{\ell}=\varphi_{\ell}$.

The case with fourfold rotational symmetry remains an interesting open problem. While we have not yet obtained a general solution, we formulate the problem mathematically. In particular, the requirement that the constant magnitude configurations be closed under the action of the PSG is quite strong. In particular, an arbitrary such configuration, $\zeta_{\ell}=\bar{\varphi} e^{i \vartheta_{\ell}}$, must map to another such configuration. Since the transformations are linear, and the $\vartheta_{\ell}$ are arbitrary, this requires that the representation matrices of the PSG elements in this basis must take the form $G=\Lambda P$, where, as we noted in Section I, $\Lambda$ is a unitary diagonal matrix (i.e. a matrix whose only non-zero elements are complex numbers of 
unit magnitude on the diagonal), and $P$ is a permutation matrix. Thus it is sufficient to determine whether the algebra of the generators of the PSG (unit $x$ and $y$ translations, and $\pi / 2$ rotations) can be faithfully represented by such matrices.

We believe that such permutative representations of the full (square symmetry) PSG exist only for specific $q$. As indicated above, we have constructed explicit examples in detail in the text for $q=2,4$. The ability to do so in these two cases is not obvious in the Landau gauge construction of the previous Sections. In Appendix C, we show that it can be clarified for $q=4$ (for $q=2$ it is rather straightforward) by choosing a different "symmetric" gauge which more closely respects the fourfold rotational symmetry of the lattice. The dual vortex theory constructed in this gauge immediately yields a permutative representation of the PSG. Generally, a permutative representation exists only for specially chosen $q$. In Appendix E, we prove that no such representation exists for $q=3$, and also give explicit examples of such representations for $q=8,9$. The latter examples are obtained from a construction which can be generalized to $q=n^{2}, 2 n^{2}$ with any integer $n$, but which we cannot guarantee yields a solution. Nonetheless, we speculate that all members of this family support a permutative representation.

For those cases for which the phase-only description can be achieved, we return to the question of charge fractionalization by considering the 'flux' (charge) of the dual 'vortices'. Supposing the constant amplitude condition, for slowly varying $\vartheta_{\ell}$ the effective phase-only action can be written

$$
S_{e f f}=\int d^{2} r d \tau \sum_{\ell} \frac{\rho_{s}^{\prime}}{2}\left|\partial_{\mu} \vartheta_{\ell}-A_{\mu}\right|^{2}+\frac{1}{2 e^{2}}\left(\epsilon_{\mu \nu \lambda} \partial_{\nu} A_{\lambda}\right)^{2},
$$

with $\rho_{s}^{\prime}=2 \bar{\varphi}^{2}$. Consider a fixed 'vortex' (independent of $\tau$ ) in one - say $\vartheta_{0}$ - of the $q$ phase fields, centered at the origin $r=0$. One has the spatial gradient $\vec{\nabla} \vartheta_{0}=\hat{\phi} / r$, all other $\vec{\vartheta}_{\ell}=0$ for $\ell \neq 0$ (here $\hat{\phi}=(-y, x) / r$ is the tangential unit vector). Clearly, the action is minimized for tangential $\vec{A}=A \hat{\phi}$. Far from the 'vortex' core, the Maxwell term $\left(\epsilon_{\mu \nu \lambda} \partial_{\nu} A_{\lambda}\right)^{2}$ is negligible, so one need minimize only the first term in Eq. 3.1. The corresponding Lagrange density at a distance $r$ from the origin is thus

$$
\mathcal{L}_{v}^{\prime}=\frac{\rho_{s}^{\prime}}{2}\left[(1 / r-A)^{2}+(q-1) A^{2}\right] .
$$

Minimizing this over $A$, one finds $A=1 /(q r)$. Integrating this to find the flux gives

$$
\oint \vec{A} \cdot d \vec{r}=\frac{2 \pi}{q} .
$$

Since the physical charge is just this dual flux divided by $2 \pi$, the 'vortex' in $\varphi_{0}$ indeed carries fractional boson charge $1 / q$.

This simple analysis can be confirmed by a straightforward but more formal duality calculation on a latticeregularized hard-spin $\vartheta_{\ell}$ model. In the continuum, the theory of the $q \varphi_{\ell}$ vortex fields coupled to the single noncompact $\mathrm{U}(1)$ gauge field $A_{\mu}$ is described by the action $\mathcal{S}_{0}+\int d^{2} x d \tau \mathcal{L}_{4}$ where $\mathcal{S}_{0}$ is as in Eq. (2.19) and $\mathcal{L}_{4}$ has the structure (in an appropriate basis which realizes the permutative PSG)

$$
\mathcal{L}_{4}=\sum_{m, n} v_{m n}\left|\varphi_{m}\right|^{2}\left|\varphi_{n}\right|^{2},
$$

where the co-efficients $v_{m n}$ are constrained by the permutative PSG; specifically, the matrix $v$ must be invariant under all the permutative transformations $P$ of the PSG: $v=P^{-1} v P$. As we have discussed above, this theory has $q-1$ globally conserved charges associated with the symmetries of phase rotations of the differences of the $\vartheta_{\ell}$ fields. The naive dual form of this continuum theory obtained ${ }^{41,42}$ from a lattice-regularized hard-spin $\vartheta_{\ell}$ model has the following continuum representation in a theory of $q$ bosons $\xi_{\ell}$ (these are the 'vortices' in the vortex field $\varphi_{\ell}$ ) each carrying boson charge $1 / q$, and $q$ non-compact $\mathrm{U}(1)$ gauge fields $\widetilde{\mathcal{A}}_{\mu}^{\ell}$ with the action

$$
\begin{aligned}
\mathcal{S}_{\xi} & =\int d^{2} x d \tau\left[\sum_{\ell=0}^{q-1}\left[\left|\left(\partial_{\mu}-i \widetilde{\mathcal{A}}_{\mu}^{\ell}\right) \xi_{\ell}\right|^{2}+\widetilde{s}\left|\xi_{\ell}\right|^{2}\right]\right. \\
& +\sum_{m, n} K_{m n}\left(\epsilon_{\mu \nu \lambda} \partial_{\nu} \widetilde{\mathcal{A}}_{\lambda}^{m}\right)\left(\epsilon_{\mu \rho \sigma} \partial_{\rho} \widetilde{\mathcal{A}}_{\sigma}^{n}\right) \\
& \left.+\widetilde{K}\left(\sum_{\ell=0}^{q-1} \widetilde{\mathcal{A}}_{\mu}^{\ell}\right)^{2}+\sum_{m, n} \widetilde{v}_{m n}\left|\xi_{m}\right|^{2}\left|\xi_{n}\right|^{2}\right] .
\end{aligned}
$$

As below Eq. (3.4), the matrices $K$ and $\widetilde{v}$ are also invariant under the permutative transformations $P$ of the PSG. Note that the 'center-of-mass' gauge field $\sum_{\ell} \widetilde{\mathcal{A}}_{\mu}^{\ell}$ is 'Higgsed' out into a massive mode by the coupling $\widetilde{K}$. It is therefore convenient to make a new choice of variables in which this 'center-of-mass' mode has been explicitly set to zero

$$
\widetilde{\mathcal{A}}_{\mu}^{\ell}=\mathcal{A}_{\mu}^{\ell}-\mathcal{A}_{\mu}^{\ell-1} .
$$

In these new variables, the $\sum_{\ell} \mathcal{A}_{\mu}^{\ell}$ gauge field is decoupled from all matter fields, and so is a redundant degree of freedom which drops out. The conserved gauge fluxes of the remaining $(q-1) \mathcal{A}_{\mu}^{\ell}$ gauge fields represent the $(q-1)$ conserved currents of the global symmetries of the $\varphi_{\ell}$ theory noted above. The field theories discussed in this paragraph have a convenient representation in 'moose' or 'quiver' diagrams ${ }^{43}$, as illustrated in Fig 9.

The following subsections will present a careful analysis of the reverse of the above duality, with special attention being paid to lattice Berry phases and symmetries.

\section{B. Fractionalization in the direct picture: $U(1)^{q-1}$ slave bosons}

Having seen that the dual $q$-vortex theory is under particular circumstances itself dual to a theory of $q$ charge 


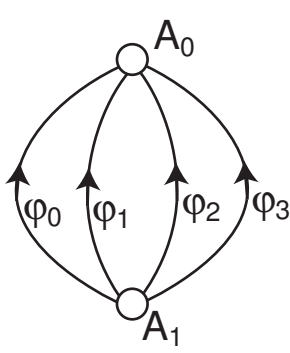

(a)

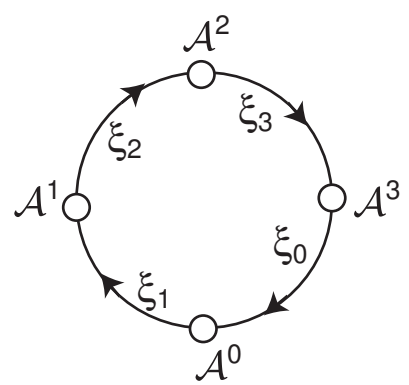

(b)
FIG. 9: Graphical representation of the field theories discussed towards the end of Section III A in "moose" diagrams for $q=4$. The circles represent non-compact $\mathrm{U}(1)$ gauge fields, while the directed lines represent complex scalar fields which are charged $+1 /-1$ under the gauge field site at their beginning/end. The $\varphi_{\ell}$ vortex theory in (a) is specified in and above Eq. (3.4) with $A_{\mu}=A_{0 \mu}-A_{1 \mu}$. Its dual theory of charge $1 / q$ bosons $\xi_{\ell}$ (which are 'vortices' in $\varphi_{\ell}$ ) in (b) is specified in Eqs. (3.5) and (3.6). In both diagrams the centerof-mass gauge field, $A_{0 \mu}+A_{1 \mu}$ or $\sum_{\ell} \mathcal{A}_{\mu}^{\ell}$, decouples from all matter fields, and is therefore immaterial. If we visualize the $A_{0} / A_{1}$ gauge fields on the north/south pole of a sphere, and the ring in (b) along the equator, then the geometric interpretation of the duality becomes evident. It is also amusing to note that the two diagrams are identical only for $q=2$, and hence only this theory is self-dual, as noted by Motrunich and Vishwanath. ${ }^{44}$

$1 / q$ bosons, it is natural to ask whether this fractionalized representation can be directly obtained from the original boson problem, without recourse to duality. We show how this is done here, and further how the $q$-vortex theory can be recovered from this route by dualizing the fractional boson theory directly.

\section{Construction of an effective gauge theory Hamiltonian}

Starting from a microscopic Hamiltonian like Eq. (2.2), one may introduce charge $1 / \tilde{q}$ slave-rotor variables, $\hat{\phi}_{i \ell}, \hat{n}_{i \ell}$, with $\left[\hat{\phi}_{i \ell}, \hat{n}_{j \ell^{\prime}}\right]=i \delta_{i j} \delta_{\ell \ell^{\prime}}$ :

$$
\begin{aligned}
e^{i \hat{\phi}_{i}} & =\prod_{\ell=0}^{\tilde{q}-1} e^{i \hat{\phi}_{i \ell}} \\
\hat{n}_{i} & =\frac{1}{\tilde{q}} \sum_{\ell=0}^{\tilde{q}-1} \hat{n}_{i \ell} .
\end{aligned}
$$

Clearly, the operator $\hat{n}_{i \ell}$ counts the number of flavor $\ell$ bosons, each of which has physical charge $1 / \tilde{q}$. This is a microscopically faithful representation of the original rotor states if we, e.g. impose the constraints

$$
\hat{n}_{i \ell}-\hat{n}_{i \ell^{\prime}}=0
$$

for all $\ell, \ell^{\prime}$. Thus, the physical state with $\hat{n}_{i}=n_{i}$ is represented by the state with all slave rotors identical $\hat{n}_{i \ell}=n_{i}$. In this subsubsection we will keep $\tilde{q}$ arbitrary, with no a priori connection to the filling $f=p / q$. This connection is made in the following subsubsection.

Eq. (3.9) expresses $\tilde{q}-1$ independent constraints, which generate the same number of independent unphysical gauge rotations of the $\hat{\phi}_{i \ell}$. That is, any rotation $\hat{\phi}_{i \ell} \rightarrow \hat{\phi}_{i \ell}+\chi_{i \ell}$ with $\sum_{\ell} \chi_{i \ell}=0$ leaves the physical boson creation/annihation operators invariant. It should be noted that the representation above has another set of discrete $\left(S_{\tilde{q}}\right)$ gauge symmetries: the $\tilde{q}$ slave rotor variables can be permuted arbitrarily and independently on each site without changing $\hat{n}_{i}$ or $\hat{\phi}_{i}$. If the constraint in Eq. (3.9) is imposed exactly on every lattice site, however, the $S_{\tilde{q}}$ gauge symmetry does not lead to any additional constraints, and it leaves the $U(1)^{\tilde{q}-1}$ constrained states invariant (i.e. these states form a trivial identity representation of $S_{\tilde{q}}$ ).

Such a microscopic slave-rotor rewriting of the problem has, unfortunately, no physical content. It becomes physical only if, on long scales, a set of renormalized effective degrees of freedom "descended" from the slave rotors become physically meaningful quasiparticles, which locally violate these hard constraints. We would like, therefore, to consider a renormalized effective theory in which the fractional rotors have some local independence. In principle such a theory can be constructed by implementing the hard constraints in a path integral formulation, and studying fluctuations around a mean-field solution of this lattice field theory. For the universal phenomenological purposes of this paper, however, this is unnecessary, and we instead prefer to proceed on conceptual grounds. Importantly, it is essential that globally, in any finite system, all quantum numbers be physical, i.e. the total number of bosons must be integer. This goal is satisfied along with local fluctuations of the fractional rotors by introducing dynamical compact $U(1)$ gauge fields:

$$
\left[\mathcal{E}_{i \alpha}^{\ell}, \mathcal{A}_{j \beta}^{\ell^{\prime}}\right]=i \delta_{i j} \delta_{\alpha \beta} \delta^{\ell \ell^{\prime}}
$$

with $\{\alpha, \beta\} \in\{x, y\}$. Here $\mathcal{E}_{i \alpha}^{\ell}$ has integer eigenvalues, and $\mathcal{A}_{i \beta}^{\ell}$ is a $2 \pi$-periodic angular variable. To keep the formalism as symmetric as possible, we have introduced $\tilde{q}$ $\left(\ell, \ell^{\prime}=0 \ldots \tilde{q}-1\right)$ such fields despite there being only $\tilde{q}-1$ independent constraints. The redundancy is eliminated by requiring

$$
\sum_{\ell=0}^{\tilde{q}-1} \overrightarrow{\mathcal{E}}_{i}^{\ell}=0 .
$$

The vector overline indicates the usual two-dimensional vector $(x, y)$ notation. With these fields, we replace Eq. (3.9) by a set of Gauss' law constraints

$$
\left[\vec{\Delta} \cdot \overrightarrow{\mathcal{E}}^{\ell}\right]_{i}=\hat{n}_{i, \ell}-\hat{n}_{i, \ell+1}
$$

where, as usual, $\hat{n}_{i, \tilde{q}}=\hat{n}_{i 0}$, and the $\vec{\Delta}$. symbol indicates a lattice divergence. If we do not allow the gauge electric flux to exit the system boundaries (or there are no 
boundaries), Gauss' theorem applied to Eq. (3.12) implies as desired that the total boson charge in the system $\left(\frac{1}{\tilde{q}} \sum_{i \ell} \hat{n}_{i \ell}\right)$ is integral. The associated effective Hamiltonian is then naïvely

$$
\begin{aligned}
\mathcal{H}_{\tilde{q}}^{0}= & -t \sum_{i \ell} \cos \left(\Delta_{\alpha} \hat{\phi}_{i \ell}-\mathcal{A}_{i \alpha}^{\ell}+\mathcal{A}_{i \alpha}^{\ell-1}\right)+u\left(\hat{n}_{i \ell}-f\right)^{2} \\
& +\frac{v}{2} \sum_{i \ell}\left|\overrightarrow{\mathcal{E}}_{i}^{\ell}\right|^{2}-K \sum_{a \ell} " \cos "\left(\vec{\Delta} \times \overrightarrow{\mathcal{A}}^{\ell}\right)_{a} .
\end{aligned}
$$

In the second line above we have added quotes around the cosine term to indicate that care must actually be taken here to ensure that the unphysical center of mass of the gauge fields $\left(\sum_{\ell} \overrightarrow{\mathcal{A}}^{\ell}\right)$ is decoupled from the other physical orthogonal linear combinations. This will be achieved in the path integral formulation to which we will soon turn through the use of a Villain potential.

We now come to a subtle and crucial point in the gauge theory formulation. Eqs. (3.12), (3.13) incorporate on long scales the gauge constraints necessary to remove the unphysical aspects of the $U(1)^{\tilde{q}-1}$ gauge redundancy. Further, the Gauss' law relations as formulated in Eq. (3.12) largely remove the $S_{\tilde{q}}$ permutation gauge symmetry. What remains of the latter is an invariance under some group of global permutations, under which

$$
\begin{aligned}
& \hat{n}_{\ell} \rightarrow \hat{n}_{P(\ell)}, \\
& \hat{\phi}_{\ell} \rightarrow \hat{\phi}_{P(\ell)},
\end{aligned}
$$

where $P(\ell)$ is a permutation on $0 \ldots \tilde{q}-1$. These permutations $P(\ell)$ are not arbitrary: only for particular choices can linear transformations of $\overrightarrow{\mathcal{A}}^{\ell}$ and $\overrightarrow{\mathcal{E}}^{\ell}$ be chosen along with Eqs. (3.14) to keep Eq. (3.13) invariant. This global permutation invariance in general depends upon the particular value of $\tilde{q}$. At a minimum, it includes all cyclic permutations (generated by the elementary cyclic permutation $P(\ell)=\ell+1$, for which $\overrightarrow{\mathcal{A}}^{\ell} \rightarrow \overrightarrow{\mathcal{A}}^{\ell+1}$ and $\overrightarrow{\mathcal{E}}^{\ell} \rightarrow \overrightarrow{\mathcal{E}}^{\ell+1}$ leaves Eqs. (3.13) invariant), but it is larger for special values of $\tilde{q}$. The permutation symmetry has been reduced to a global one since the gauge fields couple different lattice sites, so bosons can no longer be permuted locally. This permutation invariance, if the microscopic constraints, Eq. (3.9), are strictly enforced, has no consequence. In our effective theory, however, for which states violating these constraints are included, this global symmetry has meaning. Since the original microscopic boson model has no corresponding symmetry, this will lead to unphysical aspects of the effective theory if it is not corrected somehow.

An appealing and simple way to remove the permutation invariance is to add a term to the Hamiltonian which removes this symmetry. We will construct such a term, guided by a few requirements. First, it should reduce to a trivial constant if the hard constraints in Eq. (3.9) are enforced exactly. Second, it should not affect the average density of the slave bosons. Third, for reasons that will become apparent in the next subsubsection, it should enlarge the naive unit cell of the fractional boson kinetic terms. Fourth, it should allow for some projective implementation of all the symmetry operations of the lattice. The last requirement is the most non-trivial, and moreover seems somewhat at odds with the third one. As we will see, it precludes us from constructing an appropriate slave-boson theory at an arbitrary filling factor. Our tentative choice is to include a term of the form

$$
\mathcal{H}_{\tilde{q}}^{1}=-\mu_{s} \sum_{i \ell}\left(m_{i \ell}-\frac{1}{\tilde{q}}\right) \hat{n}_{i \ell} .
$$

Here $m_{i \ell}$ is an integer field defined by introducing $\tilde{q}$ sublattices (the choice of sublattices will be made later), with

$$
m_{i \ell}=\left\{\begin{array}{ll}
1 & \text { for } i \in \text { sublattice } \ell \\
0 & \text { otherwise }
\end{array} .\right.
$$

This term clearly satisfies the first three requirements, but the implementation of symmetry operations is problematic. In particular, as we have actually tried to do (requirement 3), Eq. (3.15) breaks the nä̈ve translational and rotational invariances of the original model. Indeed, a non-trivial implementation of these symmetries is possible only for special values of $\tilde{q}$ and/or lattice symmetries.

A relatively simple case is that of rectangular symmetry. In this case, it is natural to choose the set of $\tilde{q}$ sublattices by defining the $\ell^{t h}$ sublattice as the set of sites $i$ for which $i_{x}=\ell(\bmod \tilde{q})-$ just labelling sequentially columns by $\ell=0,1, \ldots \tilde{q}-1,0, \ldots$ Then translations along $y$ are trivially preserved, while an $x$ translation is implemented by translating by one lattice spacing and simultaneously cyclically permuting $\ell \rightarrow \ell+1$.

Unfortunately, this prescription fails to provide a means of implementing the $\pi / 2$ rotation operation of the square symmetry group. In general, we have been unable to find any choice of sublattices which does so for arbitrary $\tilde{q}$. In fact, the only two cases for which we have succeeded in finding a sublattice choice that fully implements the square symmetry group are $\tilde{q}=2,4$. For $\tilde{q}=2$, it is achieved by taking the two checkerboard sublattices of the square lattice. Then both $x$ and $y$ translations and $\pi / 2$ rotations about a dual lattice site must be accompanied by a (there is only one!) cyclic permutation, while a $\pi / 2$ rotation about a direct lattice site requires no permutation. For $\tilde{q}=4$, one defines the four sublattices according to

$$
i \in \text { sublattice }\left\{\begin{array}{l}
0 \text { for }\left(i_{x}, i_{y}\right)=(0,0)(\bmod 2), \\
1 \text { for }\left(i_{x}, i_{y}\right)=(1,0)(\bmod 2), \\
2 \text { for }\left(i_{x}, i_{y}\right)=(1,1)(\bmod 2), \\
3 \text { for }\left(i_{x}, i_{y}\right)=(0,1)(\bmod 2) .
\end{array} .\right.
$$

These are constructed by choosing the sites of the $2 \times 2$ square including the origin at its lower-left corner, labeling them counter-clockwise $0,1,2,3$, and translating this square vertically and horizontally to cover the plane. This can also be seen (less trivially) to preserve all symmetry operations. For instance, a $\pi / 2$ rotation about a dual lattice site should be combined with a cyclic permutation, and a unit translation in the $x$ direction is effected 
by

$$
\begin{aligned}
& \hat{n}_{i 0} \leftrightarrow \hat{n}_{i+\hat{x}, 1}, \\
& \hat{n}_{i 2} \leftrightarrow \hat{n}_{i+\hat{x}, 3}, \\
& \overrightarrow{\mathcal{E}}_{i}^{0} \rightarrow-\overrightarrow{\mathcal{E}}_{i+\hat{x}}^{0}, \\
& \overrightarrow{\mathcal{E}}_{i}^{1} \rightarrow-\overrightarrow{\mathcal{E}}_{i+\hat{x}}^{3}, \\
& \overrightarrow{\mathcal{E}}_{i}^{2} \rightarrow-\overrightarrow{\mathcal{E}}_{i+\hat{x}}^{2}, \\
& \overrightarrow{\mathcal{E}}_{i}^{3} \rightarrow-\overrightarrow{\mathcal{E}}_{i+\hat{x}}^{1},
\end{aligned}
$$

and similar relations for $\hat{\phi}_{i \ell}, \overrightarrow{\mathcal{A}}_{i}^{\ell}$. One can catalogue and verify the remaining symmetries for $\tilde{q}=4$.

Crucially, we see that it is not even possible in principle to choose a set of sublattices that is preserved under rotations for most values of $\tilde{q}$, which is a minimal requirement for successfully implementing rotational symmetry.

\section{Analysis of the effective gauge theory}

Having discussed the construction of the effective gauge theory $\mathcal{H}_{\tilde{q}}=\mathcal{H}_{\tilde{q}}^{0}+\mathcal{H}_{\tilde{q}}^{1}$ in Eqs. (3.13), (3.15), with the constraint of Eq. (3.12), we now turn to its analysis. Notably, up to this point, we have rather arbitrarily chosen to split the original boson into $\tilde{q}$ parts, without any direct reference to the filling factor. From the dual analysis of Sec. III A, we expect however that the physically appropriate fraction corresponds to $\tilde{q}=q$. The connection is as follows. Due to the presence of the staggered potential $\mu_{s}$, each of the fractional bosons moves in an environment of reduced translational symmetry, with a $\tilde{q}$-site enlarged Bravais lattice unit cell. At a filling $f=p / q$, the average number of each fractional boson per enarged unit cell is then $f \tilde{q}=p \tilde{q} / q$. The smallest amount of fractionalization then consistent with an integer number of fractional bosons per unit cell is then $\tilde{q}=q$. Intuitively, with this condition, it is possible for the fractional bosons to form a featureless Mott insulator, apart from the effect of gauge fluctuations. We therefore now focus on this case, $\tilde{q}=q$. The problem as described by $\mathcal{H}_{q}=\mathcal{H}_{q}^{0}+\mathcal{H}_{q}^{1}$ (Eqs. (3.13), (3.15)), is amenable to a simple direct analysis.

Consider first the Mott phase. In Eq. (3.13), this occurs for large $u \gg t$, for which $\hat{n}_{i \ell}$ will be approximately good quantum numbers with only only weak local fluctuations in the ground state. For a properly chosen range (fine-tuning is not necessary) of $\tilde{\mu}_{s}$, one expects $\hat{n}_{i \ell}=p m_{i \ell}$ to be a qualitatively good approximation to the ground state. ${ }^{66}$ Having determined the state of the fractional bosons, one may then study just the pure $U(1)^{q-1}$ gauge theory

$$
\mathcal{H}_{U(1)}=\frac{v}{2} \sum_{i \ell}\left|\overrightarrow{\mathcal{E}}_{i}^{\ell}\right|^{2}-K \sum_{a \ell} " \cos "\left(\vec{\Delta} \times \overrightarrow{\mathcal{A}}^{\ell}\right)_{a},
$$

with the simplified Gauss' law constraint

$$
\left[\vec{\Delta} \cdot \overrightarrow{\mathcal{E}}^{\ell}\right]_{i}=\epsilon_{i \ell}
$$

where $\epsilon_{i \ell}=p\left(m_{i, \ell}-m_{i, \ell+1}\right)$.

In arriving at Eqs. (3.19), (3.20), we have, to a first approximation, treated the Mott insulator as a deconfined $U(1)^{q-1}$ "spin liquid" with only gapped gauge-charged excitations. The particular liquid in question is characterized by the presence of $q$ gapped boson excitations and the PSG of the previous subsubsection specifying the implementation of physical symmetries. As is well-known for $q=2$, however, the deconfined (or more precisely Coulomb) phase of a pure compact $U(1)$ gauge theory in $2+1$ dimensions is unstable to confinement via the proliferation of monopole instantons, as first understood by Polyakov. This is true here as well, and the monopole unbinding will lead to the various possible density-wave orders associated with the Mott state.

To see this explicitly, one can perform a simple duality transformation for the gauge theory. In its Hamiltonian version $^{67}$, this is simply the change of variables

$$
\begin{aligned}
\mathcal{E}_{\alpha}^{\ell} & =\frac{\epsilon_{\alpha \beta} \Delta_{\beta} \chi_{\ell}}{2 \pi}+\overline{\mathcal{E}}_{\alpha}^{\ell}, \\
\frac{\vec{\Delta} \times \overrightarrow{\mathcal{A}}^{\ell}}{2 \pi} & =B^{\ell},
\end{aligned}
$$

where $\overline{\mathcal{E}}_{\alpha}^{\ell}$ is an integer-valued classical $c$-number field satisfying

$$
\left[\Delta_{\alpha} \overline{\mathcal{E}}_{\alpha}^{\ell}\right]_{i}=\epsilon_{i \ell}
$$

and $\chi_{\ell}$ is an integer scalar field. Due to the redundant extra gauge field that has been introduced, we need to enforce $\sum_{\ell} \chi_{\ell}=\sum_{\ell} \overline{\mathcal{E}}^{\ell}=0$. The former can be implemented by including a large mass term, $m^{2}\left(\sum_{\ell} \chi_{\ell}\right)^{2}$ term in $\mathcal{H}$, but for simplicity of presentation we will just keep this constraint implicit. One can verify that the new variables are canonical

$$
\left[B_{a}^{\ell}, \chi_{b}^{\ell^{\prime}}\right]=i \delta_{a b} \delta_{\ell \ell^{\prime}}
$$

This reformulates the gauge theory in terms of gaugeinvariant variables. The Hamiltonian becomes

$$
\mathcal{H}_{U(1)}=\sum_{\ell}\left[\frac{v}{8 \pi^{2}}\left|\Delta_{\alpha} \chi_{\ell}-2 \pi \epsilon_{\alpha \beta} \overline{\mathcal{E}}_{\beta}^{\ell}\right|^{2}-K^{\prime \prime} \cos ^{\prime \prime}\left(2 \pi B^{\ell}\right)\right] .
$$

To treat the theory analytically is simplest for large $K \gg v$, where fluctuations of $B^{\ell}$ are typically small. One may the naively expand the "cos" term. This approximation, however, spoils the periodicity $B^{\ell} \leftrightarrow B^{\ell}+1$, or equivalently the $2 \pi \times$ integer constraint on $\chi_{\ell}$. Hence, when making this expansion, one should include an extra term in the Hamiltonian to favor integer values of $\chi_{\ell}$. One obtains then

$$
\begin{aligned}
\mathcal{H}_{U(1)}= & \sum_{\ell}\left[\frac{\tilde{v}}{2}\left|\Delta_{\alpha} \chi_{\ell}-2 \pi \epsilon_{\alpha \beta} \overline{\mathcal{E}}_{\beta}^{\ell}\right|^{2}+\frac{\tilde{K}}{2}\left(B^{\ell}\right)^{2}\right. \\
& \left.-\lambda \cos \chi_{\ell}\right]
\end{aligned}
$$


with $\tilde{K}=(2 \pi)^{2} K, \tilde{v}=v /(2 \pi)^{2}$. It is useful now to decompose $\overline{\mathcal{E}}^{\ell}$ into transverse and longitudinal parts,

$$
\overline{\mathcal{E}}_{\alpha}^{\ell}=-\frac{\epsilon_{\alpha \beta} \Delta_{\beta} \eta_{\ell}}{2 \pi}+\Delta_{\alpha} \gamma_{\ell}
$$

Again, it is preferable to take $\sum_{\ell} \eta_{\ell}=0$. Provided one chooses $\eta_{\ell}, \gamma_{\ell}$ bounded at infinity, the latter is decoupled from $\chi_{\ell}$ and contributes only an unimportant constant to $\mathcal{H}$. Since $\chi_{\ell}$ has been promoted now to a continuous field, one can then make the shift $\chi_{\ell} \rightarrow \chi_{\ell}+\eta_{\ell}$. One obtains finally

$$
\begin{aligned}
\mathcal{H}_{U(1)}= & \sum_{a \ell}\left[\frac{\tilde{v}}{2}\left|\Delta_{\alpha} \chi_{a \ell}\right|^{2}+\frac{\tilde{K}}{2}\left(B_{a}^{\ell}\right)^{2}\right. \\
& \left.-\lambda \cos \left(\chi_{a \ell}+\eta_{a \ell}\right)\right] .
\end{aligned}
$$

This is just a set of $q 2+1$-dimensional lattice sine-Gordon theories (with the implicit constraint that the center of mass does not fluctuate, $\sum_{\ell} \chi_{\ell}=0$ ). To interpret the sine-Gordon terms, recall that $B^{\ell}$ and $\chi_{\ell}$ are canonically conjugate. Hence, the operator $e^{i \chi_{\ell}}$ generates a unit shift of $B^{\ell}$. It thus creates a single quantum of the $\ell^{t h}$ gauge flux. Based on the corresponding space-time configurations, such operators are called "monopole" (instanton) operators.

It is clear from this discussion that such monopole operators transform non-trivially under space group operations. In particular, if for a particular space group element $g$, the site $a \rightarrow g(a)$ and under the PSG $\ell \rightarrow G(\ell)$, one has

$$
e^{i \chi_{a \ell}} \rightarrow e^{i \chi_{a \ell}} e^{i\left(\eta_{g(a), G(\ell)}-\eta_{a, \ell}\right)}
$$

For large $\tilde{K} \gg \tilde{v}$, the fluctuations of $\chi_{a \ell}$ are large on short scales, and one can integrate out these short scale fluctuations ("coarse grain") perturbatively in $\lambda$. This gives a continuum theory in which the sine-Gordon terms are spatially averaged in a cumulant expansion. One expects on general grounds that all those and only those multi-monopole terms that combine to form scalars under the space group survive in the continuum theory:

$$
\mathcal{H}_{U(1)}=\int d^{2} r\left[\frac{\tilde{v}}{2}\left|\Delta_{\alpha} \chi_{\ell}\right|^{2}+\frac{\tilde{K}}{2}\left(B^{\ell}\right)^{2}+V\left(\left\{\chi_{\ell}\right\}\right)\right],
$$

where

$$
V\left(\left\{\chi_{\ell}\right\}\right)=-\sum_{n} \frac{\lambda^{n}}{n !}\left\langle\left(\sum_{\ell} \cos \left(\chi_{\ell}+\eta_{a \ell}\right)\right)^{n}\right\rangle_{a, C} .
$$

Here the angular brackets indicate (cumulant) spatial averaging of the oscillating $\eta_{a \ell}$ factors over sites $a$ of the dual lattice (it is sufficient in practice to average over the $2 q$ sites in which $\eta_{a \ell}$ takes independent values).

In general, this averaging leaves some non-vanishing 2monopole and/or 4-monopole sine-Gordon terms $\lambda_{2}, \lambda_{4}$,

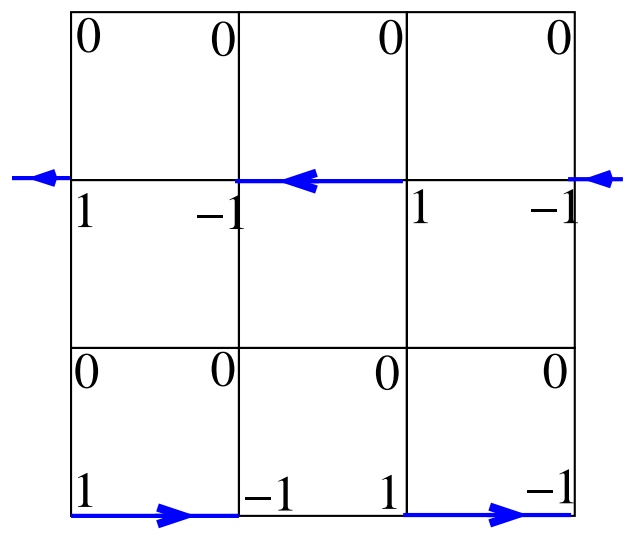

FIG. 10: Background electric field $\mathcal{E}_{i}^{0}$ configuration for $q=$ 4. The other three background fields can be obtained by rotation.

\begin{tabular}{|c|c|c|c|}
\hline$-\pi / 4$ & $\pi$ & $3 \pi / 4$ & 0 \\
\hline 0 & $3 \pi / 4$ & $\pi$ & $-\pi / 4$ \\
\hline
\end{tabular}

(3)

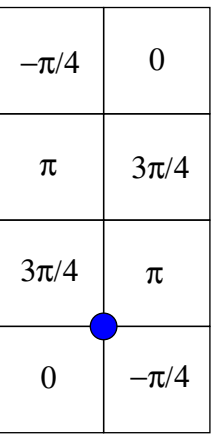

\begin{tabular}{|c|c|c|c|}
\hline $3 \pi / 4$ & $\pi$ & $-\pi / 4$ & 0 \\
\hline$\pi$ & $3 \pi / 4$ & 0 & $-\pi / 4$ \\
\hline
\end{tabular}

(1)
(0)

FIG. 11: Background scalar fields $\eta_{\ell}$ for $q=4$. Rectangular unit cells are shown for $\ell=0,1,2,3$. The filled circle indicates the origin of the direct lattice.

which depend upon the form of $\eta_{a \ell}$. As Polyakov pointed out, although the fluctuations of $\chi_{a \ell}$ are large on small scales, they are always bounded in $2+1$-dimensions, so that ultimately these non-vanishing sine-Gordon terms "pin" the $\chi_{\ell}$ fields. This has the consequence of gapping out the "photons" of the putative Coulomb phase, and furthermore, giving a non-zero average to the singlemonopole operators $e^{i \chi_{\ell}}$. According to Eq. (3.29), such averages break space group symmetries, so the resulting state necessarily has some kind of density-wave order. 
To make these manipulations concrete, we give the example of $f=1 / 4$, with the sublattice choice of Eq. (3.17). First, one must solve the Gauss' law constraint, Eq. (3.23). Then, the fields $\eta_{a \ell}$ are determined from Eqs. (3.27) by the Poisson equations,

$$
\Delta^{2} \eta_{a \ell}=2 \pi\left(\epsilon_{\alpha \beta} \Delta_{\alpha} \overline{\mathcal{E}}_{\beta}^{\ell}\right)_{a},
$$

and the requirement that they be bounded and satisfy $\sum_{\ell} \eta_{a \ell}=0 \bmod 2 \pi$. The results are shown in Figs. 10,11. For this set of $\eta_{a \ell}$, one can readily calculate the spatial averages in Eq. (3.30) up to $O\left(\lambda^{4}\right)$. One finds the effective potential takes the form

$$
\begin{aligned}
V\left(\left\{\chi_{\ell}\right\}\right)= & -\sum_{\ell}\left[\lambda_{2} \cos \left(2 \chi_{\ell}-\frac{\pi}{4}\right)+\lambda_{4} \sin 4 \chi_{\ell}\right] \\
& -\lambda_{22} \sum_{\ell<\ell^{\prime}} \cos 2\left(\chi_{\ell}-\chi_{\ell^{\prime}}\right),
\end{aligned}
$$

with $\lambda_{2} \sim \lambda^{2}, \lambda_{4}, \lambda_{22} \sim \lambda^{4}$. This indicates that for $q=4$, individual monopoles occur doubled rather than quadrupled as for $q=2$, in the partition function. They are, however, created with the non-trivial phase factor above $\left(\pi / 4\right.$ phase shift inside the cosine proportional to $\left.\lambda_{2}\right)$.

Let us now turn to the superfluid phase, occurring for $t \gg u$ in Eq. (3.13). In this case, we expect the fractionalized bosons to condense, i.e. neglecting gauge fluctuations, $\left\langle e^{i \hat{\phi}_{\ell}}\right\rangle \neq 0$. By the usual Anderson-Higgs mechanism, the $U(1)^{q-1}$ gauge fields will thereby acquire a gap. With all $q$ fractional bosons condensed, of course the physical boson is also condensed, and one has a superfluid.

What are the elementary excitations in this phase? One expects these to be $q$ flavors of vortices and antivortices, corresponding to phase windings in each of the $q$ boson fields. Despite the presence of the gauge fields, these will have a logarithmic energy cost, since due to the constraint $\sum_{\ell} \overrightarrow{\mathcal{A}}_{\ell}=0(\bmod 2 \pi)$, the gauge fields cannot screen a single vortex. Thus from this (and indeed any other) universal point of view the superfluid is completely conventional.

One can imagine, however, constructing "vortex excitons", i.e. a vortex in the $\ell^{t h}$ boson field and an antivortex in $\ell^{\prime t h}$ field. These excitations carry zero physical vorticity, and indeed can be screened by the gauge fields keeping the constraint satisfied. Most simply, a vortex in the $\ell^{t h}$ and an anti-vortex in the $(\ell+1)^{t h}$ field is screened by a $2 \pi$ (elementary) flux in the field $\overrightarrow{\mathcal{A}}^{\ell}$. Thus each vortex exciton binds an elementary gauge flux. Conversely, an elementary flux of this type will necessarily bind a vortex exciton in the superfluid phase, as the "bare" gauge flux can only penetrate the superfluid in combination with a vortex exciton (at finite energy) by the Meissner effect.

Moreover, there is no microscopic conservation law for such gauge fluxes/vortex excitons, as they carry no net vorticity. Thus there will be some amplitude for tunneling between states with different numbers of such objects.
A space-time event at which such a tunneling occurs is nothing but a monopole of the type discussed in the pure gauge theory. We have already described how to calculate the amplitudes for such processes in the preceding discussion of the insulating problem. In the superfluid state, due to this binding of monopoles to vortex excitons, one may replace the notion of a flux-changing event by the creation of a vortex exciton. Equivalently, if one thinks of the action of such a term on a configuration with one vortex present, this may also be considered as a vortex flavor-changing event.

Regardless of the language used, one may deduce from this discussion the form of the dual vortex action. In particular, it should be written in terms of $q$ vortex fields $\zeta_{q}$, describing vortices in each of the $q$ fractional bosons. By the usual considerations applicable in such a vortex description, the vortex fields should be coupled to a single non-compact $U(1)$ gauge field, $A_{\mu}$, whose 3 -curl represents the physical conserved boson current. As usual, in zero applied magnetic field, time-reversal symmetry implies symmetry between vortices and anti-vortices, so that the theory must be "particle-hole symmetric", i.e. relativistic in these variables. Provided the PSG contains enough symmetry between the different bose fields (as in all cases we have considered), the quadratic part of the vortex action is therefore fixed to have the form in Eq. (2.19). The full action $\mathcal{S}=\mathcal{S}_{0}+\int d^{2} r d \tau V\left(\zeta_{\ell}\right)$ has an additional "potential" part. One expects

$$
V\left(\zeta_{\ell}\right)=V_{0}\left(\left|\zeta_{\ell}\right|\right)+V_{\lambda}\left(\zeta_{\ell}\right)
$$

Here the first term is a vortex-conserving potential deriving from terms not involving monopole events. It can be chosen as, e.g. $V_{0}=u \sum_{\ell}\left|\zeta_{\ell}\right|^{4}$, simply to favor equally all vortex flavors. The second term is deduced from the monopole terms already derived above by the rule

$$
V_{\lambda}\left(\zeta_{\ell}\right)=\left.V\left(\left\{\chi_{\ell}\right\}\right)\right|_{e^{i \chi_{\ell} \rightarrow c \zeta_{\ell}^{*}} \zeta_{\ell+1}},
$$

the latter replacement encoding the coincidence of monopole with vortex flavor-changing events. Here $c$ is a constant associated with amplitude of $\zeta_{\ell}$. In the example of $q=4$, one can readily see that the $\lambda_{2}$ term in Eq. (3.32) leads to a term in $V_{\lambda}$ gauge-equivalent to the $\lambda$ term in Eq. (2.36).

Thus we have recovered the form of the vortex action studied in the remainder of the paper by this reasoning. Of course, rather than the physical arguments used above, we could proceed rather more mechanically by performing duality transformations both for the $U(1)$ gauge fields and for the fractional boson fields. This procedure recovers identical results, and is sketched in Appendix F.

\section{Approach to fractionalization from the density-wave state}

At half-filling, a very intuitive picture of fractionalization at the deconfined QCP could be developed based 
upon topological defects in the valence bond ordered state. In this subsection, we consider the generalization of this notion to a potential deconfined QCP at $f=1 / 4$. We will see that indeed there are intersections of particular "elementary" domain walls in the charge ordered state that can be regarded as discrete vortices. Under not very restrictive conditions, such vortices trap a fractional charge in units of $1 / 4$. Though we frame the discussion in this rather specific context, it is clear that the arguments in this subsection are rather general in nature. Indeed, we expect that it is a common (not universal but true in a sizeable fraction of cases) feature of charge ordered states breaking discrete lattice symmetries, that intersections of domain walls carry fractional charge. Of course, this does not imply in and of itself any exotic physics. Such discrete vortex configurations are certainly "confined" in the charge ordered state, having an energy linear in system size due to their trailing domain walls.

Turning now back to the specific problem of $f=1 / 4$, we first need to understand the order parameter space of the density wave state. As discussed above, the appropriate phase in question corresponds to that in region $\mathrm{E}$ of Fig. 5. From the figure, one can immediately see that the density wave has a 16 site unit cell with a period of 4 lattice spacings in both the $x$ and $y$ directions. For reference, these have the following symmetry properties (see Sec. D):

1. All 16 states are invariant under $T_{x}^{4}$ and $T_{y}^{4}$, i.e. the charge density pattern in the ground state has a period of 4 lattice constants in both $x$ - and $y$ directions.

2. The unit cell contains two inequivalent centers of $\pi / 2$ rotation, separated by two lattice spacings in the $x$ and $y$ directions from one another.

3. Reflection planes along the $x$ and $y$ axes pass through the centers of rotation.

4. All 16 states are related to each other by successive $T_{x}$ or $T_{y}$ operations.

All these symmetry properties, and the proper particle filling, can be reproduced trivially by a wavefunction taken as an exact boson number eigenstate on every site, of the form shown in Fig. 12. It is then clear that this phase may be interpreted as a charge density wave (CDW). Another caricature which is also useful to consider is a state which is a direct product of states defined on the $4 \times 4$ unit cells, with exactly 4 bosons per unit cell, but with charge fluctuations within each unit cell (see Fig. 13). More generally, the ground state of course contains further local charge fluctuations. Nevertheless, we expect that qualitative properties within the CDW phase can be determined by adiabatic continuity from the simpler caricatured states.

Clearly, the order parameter space can be specified by giving the lattice coördinates of one of the two inversion centers, i.e. an ordered pair of integers $(m, n)$ modulo

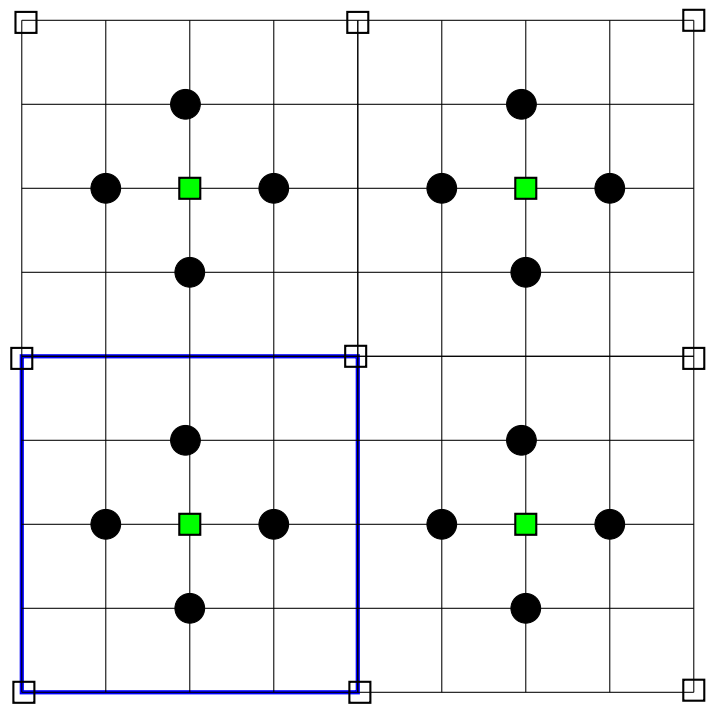

FIG. 12: Caricature of the ground state for the CDW phase at $f=1 / 4$. Here the black circles are occupied sites. A unit cell is outlined in the lower left corner. Sites on the boundary are shared amongst 2 or 4 unit cells. The squares (open and filled) indicate the two inequivalent centers of $\pi / 2$ rotations within each unit cell.

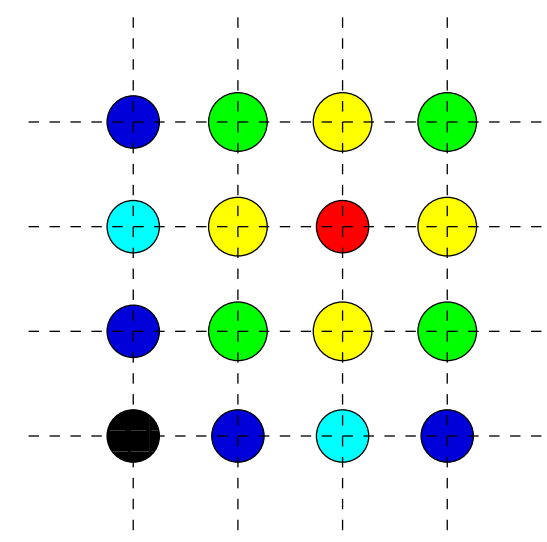

FIG. 13: A less extreme cartoon of a unit cell of the 16fold degenerate CDW ground state. Different shades of gray represent different boson densities.

4. Let us now turn to topological defects. First consider elementary domain walls, which connect "neighboring" states in this order parameter space, i.e. where the two coördinates $\left(m^{\prime}, n^{\prime}\right)$ and $(m, n)$ differ by a unit translation in $x$ or $y$. Clearly, any one state can be separated from four others in this manner. A cartoon of such an elementary domain wall, oriented for simplicity along one of the principle axes, is indicated in Fig. 14. An important question is the nature of the local excitations in the vicinity of the domain wall, assuming for simplicity that it is flat (i.e. not in a rough phase) and pinned at the boundaries, so that it has no translational degrees of freedom.. Theoretically, one may imagine that such a domain wall may be either insulating or conducting. In the simplest 


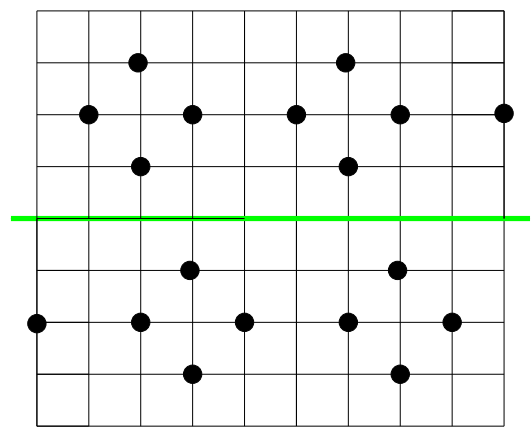

FIG. 14: Cartoon of an elementary domain wall oriented along the $x$ axis. Upon crossing from top to bottom, the state is shifted by one unit in the $x$ direction.

models, as in the cartoon of Fig. 14, the charge configuration in the vicinity of the wall is fully rigid, and one expects it to be insulating. More precisely, the excitation spectrum remains gapped in the presence of the domain wall. Moreover, one can count the excess charge (above $1 / 4$-filling) per unit length associated with the domain wall. In the caricature of Fig. 14, the excess charge is clearly vanishing. Due to the gap in the excitation spectrum, one expects this zero excess charge to be preserved as one perturbs around the caricatured state.

Henceforth we will assume that the elementary domain walls are insulating and uncharged. Now consider the nature of vortices in the CDW order parameter. Some inspiration can be obtained from the point of view of the preceding sections. We have seen that charge $\pm 1 / q$ particles correspond to $\pm 2 \pi$ vortices in each of the $\zeta_{\ell}$ fields. Thus one expects to find a total of 8 distinct such vortex-like excitations in the density wave order parameter. Indeed, by using the explicit transformations of the $\zeta_{\ell}$ fields under translations in Eqs. (C5), one can straightforwardly see that each such vortex corresponds to the intersection of four elementary domain walls. Across each such domain wall, the CDW pattern is translated to that of another of the 16 ground states. For instance, one finds that a positive vorticity vortex in $\zeta_{0}$ corresponds to the sequence of operations:

$$
T_{y}^{-1} T_{x}^{-1} T_{y} T_{x}
$$

More generally, upon moving clockwise around the center of the "vortex" in real space, one walks either clockwise or counter-clockwise in unit steps in the order parameter space. We denote these $Z_{4} \times Z_{4}$ vortices. A schematic illustration is shown in Fig. 15.

Since both the domain walls extending away from the $Z_{4} \times Z_{4}$ vortex and the bulk CDW states are gapped Mott insulators, one expects this state to have a definite and quantized charge. Again, our cartoon wavefunction gives some insight. Both the bulk and domain wall wavefunctions can be written in a form with a definite 4 bosons per $4 \times 4$ unit cell. In the $Z_{4} \times Z_{4}$ state of Fig. 15, all sites are covered by such unit cells except one site in the center of the "vortex". This fact is inevitable by symme-

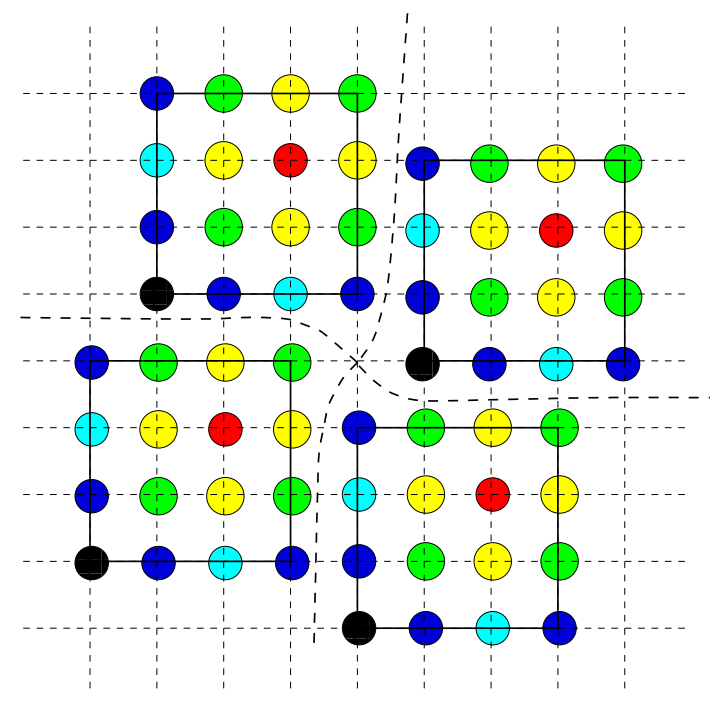

FIG. 15: A $Z_{4} \times Z_{4}$ "vortex" defect CDW phase. The site where 4 domain walls intersect does not belong to any unit cell and thus corresponds to an excitation of charge $-1 / 4$ (if empty as drawn).

try: four domains related by unit translations that can be covered by non-overlapping unit cells are related by rotation around a site of the direct lattice. Thus one can write two such caricatured wavefunctions, having either zero or one boson on this central site. As the occupancy per site in the bulk is $f=1 / 4$, such states have, relative to the ground state, a definite relative charge of $-1 / 4$ (unoccupied central site) or $+3 / 4$ (occupied central site). Because both the bulk and domain walls have been assumed insulating, we expect those quantized charges to be a robust feature of the $Z_{4} \times Z_{4}$ vortex, even for more realistic wavefunctions. This is because, although some charge may "leak" away from the central site, it cannot escape through the insulating environs, so the total excess charge summed over the region around the vortex will not change.

It is notable that one does not obtain in this way two symmetry-related states with charges $Q= \pm 1 / 4$. The difference from the case of $f=1 / 2$ is that there is no particle-hole symmetric limit corresponding to $f=1 / 4$. Indeed, there is no symmetry relating the charge $Q=$ $-1 / 4$ and $q=+3 / 4$ states, and generally these will have different energy. Of course, such a strongly localized form of the wavefunctions can be even approximately appropriate only deep in the CDW state, where any particlehole symmetry is very strongly broken. Thus there is no intrinsic contradiction with the expectations of the preceding considerations of this section. Of course, deep in the CDW phase such a state has an energy linearly diverging with system size, due to the four domain walls extending to the system boundaries. Its emergence as a deconfined "particle" 68 in the spectrum near the critical point is not at all obvious from this perspective.

Apparently if a deconfined QCP obtains in this case, 
particle-hole symmetry must be at least approximately restored in its vicinity, which is remarkable from this point of view. Its absence deep in the CDW phase, while not intrinsically troubling, makes an attempt to formulate a theory of the QCP directly in terms of such microscopic vortex defects not very promising. We therefore leave it an open subject for future work.

\section{IMPURITY PINNING}

This section will show how our approach offers a natural framework for describing recent STM observations $^{4-8,19}$ of modulations in the local density of states of the cuprate superconductors. As we noted in Section I and demonstrated in II, the bosonic theories presented so far apply also to paired electron systems, with the PSG determined in Eq. (1.6) by the density of Cooper pairs. We will interpret the observed density STM modulations as representing density wave order associated with a proximate superfluid-to-insulator transition. Indeed, as we have discussed in Section I, the PSG requires density wave order to be present near all such transitions in which the elementary vortices do not form composites.

As we will demonstrate below, an important advantage of our focus on the $\varphi_{\ell}$ vortex degrees of freedom, and their connection to density wave modulations, is that we are able to use the same theory to compute the impurity-induced pinning of density wave order in zero magnetic field, and also the vortex-induced density wave order in a finite magnetic field. At zero magnetic field, there are vacuum fluctuations of vortex-anti-vortex pairs which, when pinned by impurities, lead to modulations observable in STM. At finite magnetic field, there is a net excess of vortices over anti-vortices (say) but precisely the same pinning potentials again lead to density wave order. This section will consider a simple toy model of the pinning process, with the aim of exposing the very general relationship between the zero field and finite field STM experiments.

The perspective offered here differs considerably from previous analyses of the STM experiments ${ }^{46-55}$ (although VBS order associated with vortices was suggested prior to the experiments in Ref. 56). In Refs. 46-48 the spin and charge order were treated as the primary fluctuating degrees of freedom which were pinned by localized impurities. However, the connection of this pinning to the location of the vortices was indirect, and in a sense, had to be put in by hand. It was assumed that a large coupling $v_{|\mathbf{Q}|}$ in Eq. (1.5) caused each vortex to induce a large pinning potential for the fluctuating density wave order. Our present approach dispenses with this ad hoc procedure, and shows how the connection between vorticity and density wave order emerges directly from the underlying quantum physics.

We will work within the continuum field theory of low energy vortex fluctuations in the superfluid discussed in
Section II C. As our purpose is mainly illustrative, we will only consider this theory within a leading Gaussian approximation; our approach can, in principle, be extended to include the non-linear terms discussed in Section II C using the formalism presented below. However, the gauge potential $A_{\mu}$ has to be considered with some care, and cannot naïvely be dropped: we have to include an average value of $A_{\tau}$ which accounts both for the vortex "chemical potential" (proportional to the applied physical magnetic field) and for the interaction between vortices in the vortex lattice. We therefore consider the following action for the $\varphi_{\ell}$ vortices

$$
\begin{gathered}
\mathcal{S}_{0}=\int d^{2} r d \tau \sum_{\ell=0}^{q-1}\left[\left|\left(\partial_{\tau}-i \bar{A}_{\tau}(\mathbf{r})\right) \varphi_{\ell}\right|^{2}+c^{2}\left|\partial_{\alpha} \varphi_{\ell}\right|^{2}\right. \\
\left.+m_{v}^{2} c^{4}\left|\varphi_{\ell}\right|^{2}\right]
\end{gathered}
$$

where the index $\alpha$ extends over the two spatial directions. This is clearly derived from the action in Eq. (2.19), written in a notation convenient for our purposes here: the energy gap towards creation of a vortex or anti-vortex is $m_{v} c^{2}$, and $c$ is a velocity. Translational invariance is broken by adding a pinning potential: we assume a static impurity potential which couples linearly to the density operator:

$$
\mathcal{S}_{V}=\int d^{2} r d \tau \sum_{m n} V_{m n}(\mathbf{r}) \rho_{m n}^{*}(\mathbf{r}, \tau)
$$

where $\rho_{m n}$ is specified as in Eq. (1.15). Since $\rho_{m n}^{*}(\mathbf{r}, \tau)=$ $\rho_{-m,-n}(\mathbf{r}, \tau)$ we must have $V_{m n}^{*}(\mathbf{r})=V_{-m,-n}(\mathbf{r})$. We will now consider the properties of the action $\mathcal{S}_{0}+\mathcal{S}_{V}$ in zero and non-zero magnetic field in turn in the following subsections.

\section{A. Pinning in zero magnetic field}

In this case we can set $\bar{A}_{\tau}=0$, and perform a perturbative expansion in powers of $V_{m n}$. A one-loop computation of the $\varphi_{\ell}$ susceptibility shows

$$
\left\langle\rho_{m n}(\mathbf{r})\right\rangle=S\left(\left|\mathbf{Q}_{m n}\right|\right)^{2} \int d^{2} r^{\prime} \Pi_{0}\left(\mathbf{r}-\mathbf{r}^{\prime}\right) V_{m n}\left(\mathbf{r}^{\prime}\right)
$$


where the susceptibility is

$$
\begin{aligned}
\Pi_{0}(\mathbf{r})= & -q \int \frac{d^{2} k}{4 \pi^{2}} \frac{d^{2} p}{4 \pi^{2}} \frac{d \omega}{2 \pi} \frac{e^{i \mathbf{k} \cdot \mathbf{r}}}{\left(\omega^{2}+p^{2} c^{2}+m_{v}^{2} c^{4}\right)} \\
& \times \frac{1}{\left(\omega^{2}+(\mathbf{k}+\mathbf{p})^{2} c^{2}+m_{v}^{2} c^{4}\right)} \\
= & -q \int \frac{d^{2} k}{4 \pi^{2}} \frac{e^{i \mathbf{k} \cdot \mathbf{r}}}{4 \pi c^{3} k} \tan ^{-1}\left(\frac{k}{2 m_{v} c}\right) \\
= & -\frac{q}{8 \pi^{2} c^{3}} \int_{0}^{\infty} d k J_{0}(k r) \tan ^{-1}\left(\frac{k}{2 m_{v} c}\right) \\
= & -\frac{q m_{v}}{4 \pi^{2} c^{2}} \int_{1}^{\infty} d y \int_{0}^{\infty} \frac{k d k J_{0}\left(2 m_{v} c r k\right)}{\left(y^{2}+k^{2}\right)} \\
= & -\frac{q m_{v}}{4 \pi^{2} c^{2}} \int_{1}^{\infty} d y K_{0}\left(2 m_{v} r c y\right) \\
\approx & -\frac{q m_{v}}{4 \pi^{2} c^{2}} \sqrt{\frac{\pi}{2}} \frac{e^{-2 m_{v} c r}}{\left(2 m_{v} c r\right)^{3 / 2}} \text { as } r \rightarrow \infty .
\end{aligned}
$$

So the pinned order decays exponentially with $r$ over the length scale $1 /\left(2 m_{v} c\right)$.

\section{B. Pinning in a non-zero magnetic field}

A non-zero magnetic field induces a finite density of vortices, and consequently we have to consider the theory in the presence of a non-zero vortex "chemical potential". Here we wish to focus on the behavior of a single vortex localized near the origin, surrounded by an infinite vortex lattice. In this case, the quantity $\bar{A}_{\tau}$ has two distinct contributions: $(i)$ a spatially independent vortex chemical potential, $\mu_{v}$, proportional to the applied magnetic field, and (ii) a $\mathbf{r}$ dependent potential created by the surrounding vortices of the vortex lattice. The latter potential arises from the superflow around each vortex: in the present dual theory it is the logarithmic "Coulomb" interaction energy associated with the $A_{\mu}$ gauge field created by the total vortex "charge" density. For the vortex under consideration, this "Coulomb" potential is assumed to have a minimum near $\mathbf{r}=0$, and we can safely perform a quadratic expansion of the potential around its minimum. Collecting these contributions, we write

$$
i \bar{A}_{\tau}(\mathbf{r})=-\mu_{v}+\frac{1}{2} m_{v} \omega_{v}^{2} r^{2} .
$$

The second contribution above represents the interaction energy between the vortices, and the frequency $\omega_{v}$ is determined by the superfluid stiffness of the bosons, and the spacing of the vortex lattice. At a more sophisticated level, we really have to consider the collective oscillations of the entire vortex lattice, and quantize the motion of all the vortices together ${ }^{57}$; however, we will be satisfied here with the simple 'Einstein model' of the vortex lattice oscillation spectrum which is implicit in Eq. (4.5).

A finite density of vortices requires that the vortex chemical potential be larger than the energy gap to create a vortex. Hence we have $\mu_{v}>m_{v} c^{2}$. In the analytic computations of this subsection, we will consider here only the additional density modulation created by the vortices, while neglecting the 'vacuum' quantum fluctuations of the vortex-anti-vortex pairs; provided $\left|i \bar{A}_{\tau}-m_{v} c^{2}\right| \ll m_{v} c^{2}$, these 'vacuum' polarization contributions can be assumed to yield an additive contribution to the density modulation equal to that already computed in Section IV A. Technically, we can ignore the vortex-anti-vortex pairs simply by performing a low frequency expansion of Eq. (4.1) in the presence of a nonzero $\mu_{v}$. To this end we define

$$
\varphi_{\ell}=\frac{1}{\sqrt{2 i \bar{A}_{\tau}}} \Psi_{\ell} \approx \frac{1}{\sqrt{2 m_{v} c^{2}}} \Psi_{\ell},
$$

and expand Eq. (4.1) to leading order in temporal derivatives and in $\left|i \bar{A}_{\tau}-m_{v} c^{2}\right|$. This yields the following familiar action for a bosonic vortex "particle" with $q$ 'flavors' $\Psi_{\ell}$

$$
\begin{aligned}
\mathcal{S}_{0}=\int & d^{2} r d \tau \sum_{\ell=0}^{q-1}\left[\Psi_{\ell}^{*} \frac{\partial \Psi_{\ell}}{\partial \tau}+\frac{\left|\partial_{\alpha} \Psi_{\ell}\right|^{2}}{2 m_{v}}\right. \\
& \left.+\left(m_{v} c^{2}-\mu_{v}+\frac{1}{2} m_{v} \omega_{v}^{2} r^{2}\right)\left|\Psi_{\ell}\right|^{2}\right]
\end{aligned}
$$

We also have to insert Eq. (4.6) into Eq. (4.2) and so obtain the pinning potential acting on the vortices: this potential has a non-trivial structure in the 'flavor' space of the $q$ vortices, and this will naturally be crucial in determining the induced density wave modulation.

Just as in Section IV A, we proceed in a perturbative expansion in powers of $V_{m n}$. However, unlike the previous zero magnetic field case, we now find that the perturbation theory is degenerate, and remarkably the leading order result turns out to be independent of the overall scale of the $\left|V_{m n}\right|$. To zeroth order in the $V_{m n}$, notice that the vortex particle described by Eq. (4.7) has a $q$ fold degenerate ground state, with its spatial wavefunction given by the ground state of the harmonic oscillator

$$
\Psi_{\ell}(\mathbf{r})=\mathcal{U}_{\ell}\left(\frac{m_{v} \omega_{v}}{\pi}\right)^{1 / 2} \exp \left(-\frac{m_{v} \omega_{v} r^{2}}{2}\right)
$$

where $\mathcal{U}_{\ell}$ is, for now, an arbitrary unit vector in the internal vortex space. The $\mathcal{U}_{\ell}$ vector is determined by diagonalizing the pinning potential projected into this degenerate ground state manifold. From Eqs. (4.2) and (1.15) we deduce that $\mathcal{U}_{\ell}$ is the ground state eigenvector of the matrix $\mathcal{M}_{\ell m}$,

$$
\sum_{m} \mathcal{M}_{\ell m} \mathcal{U}_{m}=\epsilon_{0} \mathcal{U}_{\ell}
$$

where

$$
\begin{aligned}
& \mathcal{M}_{\ell m}=\frac{\omega_{v}}{2 \pi c^{2}} \int d^{2} r \exp \left(-m_{v} \omega_{v} r^{2}\right) \\
& \times \sum_{n}\left[S\left(\left|\mathbf{Q}_{n, \ell-m}\right|\right) V_{n, \ell-m}(\mathbf{r}) \omega^{-n(\ell+m) / 2}\right] .
\end{aligned}
$$


Note that the overall scale of $\mathcal{M}_{\ell m}$ is immaterial at this order in perturbation theory because it has no influence on $\mathcal{U}_{\ell}$. Finally, the density wave order associated with this vortex is determined by inserting Eqs. (4.6) and (4.8) into Eq. (1.15):

$$
\begin{aligned}
\left\langle\rho_{m n}(\mathbf{r})\right\rangle=\frac{S\left(\left|\mathbf{Q}_{m n}\right|\right) \omega_{v}}{2 \pi c^{2}} \exp \left(-m_{v} \omega_{v} r^{2}\right) \\
\times \omega^{m n / 2} \sum_{\ell} \mathcal{U}_{\ell}^{*} \mathcal{U}_{\ell+n} \omega^{\ell m} .
\end{aligned}
$$

As promised, this result has no prefactor of $V_{m n}$ : the dependence on the pinning potential is entirely in Eqs. (4.9) and (4.10) where the relative values of $V_{m n}$ determine the orientation of the unit vector $\mathcal{U}_{\ell}$ in the complex $q$ dimensional vortex space.

We also note here that the above calculation can be extended to allow for small deviations from the commensurate density of $p / q$. We do this by extending Eq. (4.1) as in Eq. (2.46), and include an average dual 'magnetic' field of strength $2 \pi \delta f / a^{2}$ that acts on the vortices. However, it cannot be assumed that the dual 'magnetic' field is spatially uniform, and its space dependence must be computed self-consistently. We expect that the dual 'magnetic' flux will be partially expelled from the vicinity of the vortices (where the dual 'matter' resides). In the direct boson language, this means that the density of bosons is not spatially uniform in the presence of the vortex lattice, and that the boson density is closer to $p / q$ than average at the positions of the vortex cores. This effect has been discussed in Ref. 58 for the case of Mott insulators with integer filling. Let us assume that the actual boson density deviation from commensurability at the vortex lattice positions is $\delta \widetilde{f}$, where $|\delta \widetilde{f}|<|\delta f|$. We now need to determine the vortex wavevfunction by solving the Schrödinger equation associated with Eq. (4.7) and a 'magnetic' field of strength $\delta \widetilde{f}$. This is equivalent to the problem of a particle in a parabolic potential and a magnetic field; the wavefunction of the vortex retains the Gaussian form in Eq. (4.8), and all subsequent expressions remain as in the paragraphs above apart from the shift in the frequency $\omega_{v} \rightarrow\left(\omega_{v}^{2}+\left(2 \pi \delta \widetilde{f} /\left(2 m_{v} a^{2}\right)\right)^{2}\right)^{1 / 2}$.

\section{Discussion}

The above computations of pinned density wave order in zero and non-zero magnetic field have a number of unusual features which deserve further comment. It would be useful to have tests of these features in future STM experiments.

The pinned order in zero field in Eq. (4.4) has a conventional form similar to that obtained in other approaches. The induced order decays exponentially from the pinning site, on a scale $1 /\left(2 m_{v} c\right)$ related to the distance to the superfluid-to-insulator transition. This decay length equals the intrinsic correlation length for density wave order fluctuations in the superfluid, as might naïvely be expected. Also, for weak pinning, the amplitude of the pinned order is proportional to the strength of the pinning.

In contrast, the finite magnetic field result in Eq. (4.11) has some remarkable features. Quite generally, for weak pinning, the envelope of the density wave order has the functional form of a Gaussian in the small $r$ region where the harmonic approximation holds. The width of this Gaussian is not determined by the correlation length of the density wave order, but by the scale over which the vortex undergoes quantum zero point motion. The latter distance is determined by the inverse square root of the mass of the vortex, $m_{v}$, and the vortex lattice oscillation frequency, $\omega_{v}$. It should be possible to compute the magnetic field dependence of $\omega_{v}$, and thus quantitatively compare Eq. (4.11) with STM experiments in a varying magnetic field. Finally, the prefactor of Eq. (4.11) is independent of the strength of the pinning field, but has an interesting dependence on $\omega_{v}$, which places further constraints on possible experimental comparisons; the independence on the pinning field strength can readily tested in numerical studies such as those in Refs. 36, 59, and 60.

\section{CONCLUSIONS}

This paper has presented a general framework for describing the superfluid-to-insulator transition on regular lattices. We examined simple model boson systems on the square lattice, but we expect our results to be quite general, and applicable also to paired electron systems exhibiting transitions between superconducting and Mott insulating states. The latter connection is established in more detail in a companion paper $^{20}$ II.

There were three important themes underlying our approach. The first is a feature common to several recent studies of quantum phase transitions with multiple order parameters ${ }^{13,15,16,45}$ : the quantum mechanics of the defects of the order parameter in one phase induce correlations of the second phase. In our case, we focused on the vortex defects of the superfluid state. These were found to have remarkable symmetry properties which linked them intricately to density wave order. The condensation of such vortices, apart from leading to the destruction of superfluid order, also led to the simultaneous appearance of density wave order in the Mott insulator. This constitutes an important mechanism for the breakdown of the Landau-Ginzburg-Wilson theory of the phase transition.

The second theme was the crucial role played by projective representations of the space group of the lattice (the PSGs). PSGs have been highlighted in the recent work of $\mathrm{Wen}^{31}$ in his study of ground states insulating spin systems (equivalently, of bosons at half-filling). We argued that the vortices of the superfluid in the vicinity of a commensurate Mott insulator state transform under a PSG defined by Eqs. (1.8) and (1.10). The key defining 
parameter of this PSG is the unimodular complex number $\omega$, and for a Mott insulator with average density of $p / q$ bosons per lattice site $\omega$ was defined in Eqs. (1.9) and (1.11). Note that the superfluid (or a supersolid phase, if present) need not be exactly at this density, and our formalism allows small variations in density away from that of the commensurate Mott insulator (see Section IIF). The existence of such a PSG was shown to imply that there are $q$ degenerate species of vortices in the superfluid. Moreover, we argued that any linear superposition of such vortices was necessarily associated with density wave order at wavelengths which are integer divisors of $q$ lattice spacing: consequently, as noted above, vortex condensation produces a Mott insulator with long-range density wave order.

Section IV presented an analysis of the consequences of impurities in the superfluid. Any impurity breaks the lattice symmetry, and hence it also lifts the degeneracy between the $q$ vortex species. It then follows that any localized vortex has an associated halo of density wave order, and we offered this as the fundamental explanation of the STM observations of Hoffman et al. ${ }^{19}$ on $\mathrm{Bi}_{2} \mathrm{Sr}_{2} \mathrm{CaCu}_{2} \mathrm{O}_{8+\delta}$.

The third theme of our paper was one of particle number fractionalization. This arose in an attempt to interpret the dual vortex theory of the LGW-forbidden superfluid-insulator transition in the language of the direct lattice bosons. Following the 'deconfined criticality' proposal of Senthil et al. ${ }^{15}$, we hypothesized a theory in which the boson fractionalized into $q$ constituent particles each with particle number $1 / q$. By performing a duality analysis upon such a fractionalized boson theory, we were able to reproduce the dual theory of $q$ vortices, but only for special values of $q$. The selected values of $q$ were those for which it was possible to obtain a permutative representation of the PSG.

Moving beyond simple boson systems, our analysis makes clear that the key ingredient needed for other superfluids is the value of the parameter $\omega$ controlling the PSG of its vortices. With the knowledge of $\omega$, the general structure of the field theory in Section II C is fully determined, and subsequent results follow. We invite the reader to now proceed to II, where we consider electron systems with short range pairing, and show that $\omega$ is determined by the density of Cooper pairs.

\section{Acknowledgments}

We thank M. P. A. Fisher, T. Senthil, Z. Tešanović, and J. Zaanen for valuable discussions. This research was supported by the National Science Foundation under grants DMR-9985255 (L. Balents), DMR-0098226 (S.S.), and DMR-0210790, PHY-9907949 at the Kavli Institute for Theoretical Physics (S.S.), the Packard Foundation (L. Balents), the Deutsche Forschungsgemeinschaft under grant BA 2263/1-1 (L. Bartosch), and the John Simon Guggenheim Memorial Foundation (S.S.). S.S. thanks the Aspen Center of Physics for hospitality. K.S. thanks S. M. Girvin for support through ARO grant 1015164.2.J00113.627012.

\section{APPENDIX A: VORTICITY MODULATIONS}

We have shown in the body of the paper that the vortex fields $\varphi_{\ell}$ are naturally connected to modulations in the generalized density (which could be any observable invariant under time reversal and spin rotations); explicitly, the two were connected by the formula in Eq. (1.15). In this appendix we will examine the issue of the modulations in the vorticity.

Following Ivanov et al. ${ }^{33}$, we define the vorticity as a observable associated with each plaquette of the direct lattice (or each site of the dual lattice), equal to the sum of the particle (boson) currents flowing anticlockwise around the plaquette. The vorticity is odd under time-reversal, and because all the states considered in the body of the paper are time-reversal invariant, its expectation value vanishes. However, when a magnetic field is applied, as in Section IV B, then the vorticity can have quite a rich spatial structure. In the superfluid phase, we know that the magnetic field induces a lattice of vortices. We also argued in Section IV B that there is a density modulation superimposed on this vortex lattice described by Eq. (4.11). Here we shall show that there is also a corresponding pattern of vorticity modulations at the wavevectors $\mathbf{Q}_{m n}$. Thus the appearance of "staggered vorticity" is quite a general feature of superfluids near a Mott transition, and not a special characteristic of certain spin liquids as implied by Ivanov et al. ${ }^{33}$.

We can obtain an expression for the vorticity either by explicit derivation or by symmetry considerations alone. In the first approach, we notice that the vorticity is conjugate to a magnetic field applied on the plaquette of a dual lattice, and the latter couples to the operator $\psi_{a}^{*} \partial_{\tau} \psi_{a}-\partial_{\tau} \psi_{a}^{*} \psi_{a}$. In the second approach, we need the action of the time-reversal operation, $\mathcal{T}$, on the varies fields in the paper; a simple analysis shows that under $\mathcal{T}$

$$
\begin{gathered}
\tau \rightarrow-\tau ; x \rightarrow x \quad ; y \rightarrow y \quad ; \hat{\phi} \rightarrow-\hat{\phi} ; \\
J_{\tau} \rightarrow J_{\tau} ; J_{x} \rightarrow-J_{x} ; J_{y} \rightarrow-J_{y} ; \\
A_{\tau} \rightarrow-A_{\tau} \quad ; A_{x} \rightarrow A_{x} ; A_{y} \rightarrow A_{y} ; \\
\psi \rightarrow \psi ; \varphi_{\ell} \rightarrow \varphi_{\ell} .
\end{gathered}
$$

From these mappings we can construct the simplest observable which is odd under $\mathcal{T}, I_{x}^{\text {dual }}, I_{y}^{\text {dual }}$, and transforms like Eq. (1.16) under translations and lattice rotations. We define $V_{m n}$ to be the Fourier component of the vorticity at the wavevector $\mathbf{Q}_{m n}$ defined in Eq. (1.14), and obtain (compare Eq. (1.15))

$$
\begin{aligned}
& V_{m n}=S_{V}\left(\left|\mathbf{Q}_{m n}\right|\right) \omega^{m n / 2} \sum_{\ell=0}^{q-1} \omega^{\ell m} \\
& \times\left(\varphi_{\ell}^{*} \frac{\partial \varphi_{\ell+n}}{\partial \tau}-\frac{\partial \varphi_{\ell}^{*}}{\partial \tau} \varphi_{\ell+n}\right) .
\end{aligned}
$$


Here $S_{V}(Q)$ is an unknown structure factor which depends smoothly on $Q$.

We can now easily extend the discussion in Section IV B to obtain the mean vorticity modulation in the vortex lattice. The analog of the result in Eq. (4.11) is

$$
\begin{gathered}
\left\langle V_{m n}(\mathbf{r})\right\rangle=\frac{S_{V}\left(\left|\mathbf{Q}_{m n}\right|\right) m_{v} \omega_{v}}{\pi} \exp \left(-m_{v} \omega_{v} r^{2}\right) \\
\times \omega^{m n / 2} \sum_{\ell} \mathcal{U}_{\ell}^{*} \mathcal{U}_{\ell+n} \omega^{\ell m} .
\end{gathered}
$$

\section{APPENDIX B: OTHER LATTICES}

We illustrate the extension of the PSG to other lattices by considering the case of bosons on a honeycomb lattice. We consider boson density $f$ per site, and as in the case of a square lattice we write $f=p / q$, with $p$ and $q$ relatively prime integers and define $\omega=e^{2 \pi i f}$. The vortices reside on the dual triangular lattice, and so we have to consider the PSG of motion on a triangular lattice in the presence of a 'magnetic' field.

The triangular lattice is spanned by the two basis vectors

$$
\mathbf{a}_{1}=\mathbf{e}_{x}, \quad \mathbf{a}_{2}=-\frac{1}{2} \mathbf{e}_{x}+\frac{\sqrt{3}}{2} \mathbf{e}_{y} .
$$

Here $\mathbf{e}_{x}$ and $\mathbf{e}_{y}$ are orthogonal unit vectors. Any position vector of the lattice can be written as $\mathbf{a}=a_{1} \mathbf{a}_{1}+a_{2} \mathbf{a}_{2}$ with $a_{1}$ and $a_{2}$ integers. It is also convenient to define

$$
\mathbf{a}_{d}=\mathbf{a}_{1}+\mathbf{a}_{2}=\frac{1}{2} \mathbf{e}_{x}+\frac{\sqrt{3}}{2} \mathbf{e}_{y} .
$$

Here we will use the Landau gauge $\bar{A}_{a \tau}=\bar{A}_{a 1}=0$ and

$$
\bar{A}_{a 2}=2 f a_{1}, \quad \bar{A}_{a d}=f\left(2 a_{1}+1\right) .
$$

For the translation and rotation operators we obtain

$$
\begin{aligned}
T_{1}: \psi\left(a_{1}, a_{2}\right) & \rightarrow \psi\left(a_{1}-1, a_{2}\right) \omega^{2 a_{2}} \\
T_{2}: \psi\left(a_{1}, a_{2}\right) & \rightarrow \psi\left(a_{1}, a_{2}-1\right) \\
T_{d}: \psi\left(a_{1}, a_{2}\right) & \rightarrow \psi\left(a_{1}-1, a_{2}-1\right) \omega^{2 a_{2}-1} \\
R_{\pi / 3}^{\text {dual }}: \psi\left(a_{1}, a_{2}\right) & \rightarrow \psi\left(a_{2}, a_{2}-a_{1}\right) \omega^{2 a_{1} a_{2}-a_{2}^{2}} .
\end{aligned}
$$

As in the case of a square lattice, the translation operators do not commute, and in addition to $T_{1} T_{2}=\omega^{2} T_{2} T_{1}$ we have

$$
T_{1} T_{2}=\omega T_{d} .
$$

As expected, the following relations of the triangular lattice are satisfied,

$$
\begin{aligned}
T_{1} R_{\pi / 3}^{\text {dual }} & =R_{\pi / 3}^{\text {dual }} T_{2}^{-1} \\
T_{2} R_{\pi / 3}^{\text {dual }} & =R_{\pi / 3}^{\text {dual }} T_{d} \\
T_{d} R_{\pi / 3}^{\text {dual }} & =R_{\pi / 3}^{\text {dual }} T_{1} \\
\left(R_{\pi / 3}^{\text {dual }}\right)^{6} & =1
\end{aligned}
$$

To discuss the translation and rotation operations in momentum space it is convenient to introduce the reciprocal lattice to our triangular lattice. The reciprocal lattice is also a triangular lattice which, with respect to the original (dual) triangular lattice, is rotated by 30 degrees and spanned by the basis vectors

$$
\mathbf{b}_{1}=\mathbf{e}_{x}+\frac{1}{\sqrt{3}} \mathbf{e}_{y}, \quad \mathbf{b}_{2}=\frac{2}{\sqrt{3}} \mathbf{e}_{y} .
$$

We can now write any wave-vector $\mathbf{k}$ as $\mathbf{k}=k_{1} \mathbf{b}_{1}+k_{2} \mathbf{b}_{2}$. Using $\mathbf{a}_{i} \cdot \mathbf{b}_{j}=\delta_{i j}$ we have $\mathbf{k} \cdot \mathbf{a}=k_{1} a_{1}+k_{2} a_{2}$ and obtain for the translation and rotation operations in momentum space

$$
\begin{aligned}
& T_{1}: \psi\left(k_{1}, k_{2}\right) \rightarrow \psi\left(k_{1}, k_{2}-4 \pi f\right) e^{-i k_{1}} \\
& T_{2}: \psi\left(k_{1}, k_{2}\right) \rightarrow \psi\left(k_{1}, k_{2}\right) e^{-i k_{2}} \\
& T_{d}: \psi\left(k_{1}, k_{2}\right) \rightarrow \psi\left(k_{1}, k_{2}-4 \pi f\right) \omega e^{-i k_{1}-i k_{2}} \\
& R_{\pi / 3}^{\text {dual }}: \psi\left(k_{1}, k_{2}\right) \rightarrow \\
& \frac{1}{q} \sum_{m, n=0}^{q-1} \psi\left(k_{1}+k_{2}+4 \pi f n,\right. \\
& \left.-k_{1}-4 \pi m f\right) \omega^{m(m-2 n)} \quad, q=2 \nu+1 \\
& \frac{2}{q} \sum_{m, n=0}^{q / 2-1} \psi\left(k_{1}+k_{2}+4 \pi f n,\right. \\
& \left.-k_{1}-4 \pi m f\right) \omega^{m(m-2 n)} \quad, q=4 \nu \\
& \frac{2}{q} \sum_{m, n=0}^{q / 2-1} \psi\left(k_{1}+k_{2}+4 \pi f(n-1 / 2),\right. \\
& \left.-k_{1}-4 \pi m f\right) \omega^{m(m-2 n+1)} \quad, q=4 \nu+2,
\end{aligned}
$$

where $\nu$ is an integer. Note that we find a different transformation form for the rotations depending upon the value of $q(\bmod 4)$. There is also a compact expression for the rotation operator valid for general $q$, but this obscures the underlying symmetries by containing terms which cancel each other in the case of even $q$.

As in the case of the square lattice in Section IIB, let us now consider the important vortex fluctuations at momenta where the spectrum has minima. If $|\Lambda\rangle$ is a state at a minimum of the spectrum with $T_{2}|\Lambda\rangle=e^{-i k_{2}^{*}}$, then acting successively with $T_{1}$ on this state gives us

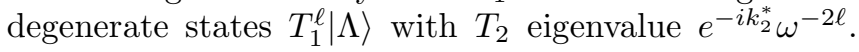
This procedure actually does not completely span the degenerate manifold for $q=4 \nu+2$, and we will limit our considerations for now to the other cases.

$$
\text { 1. } q=2 \nu+1
$$

For odd $q$ the eigenvalues $e^{-i k_{2}^{*}} \omega^{-2 \ell}$ are all different, and so the degeneracy of the vortex fields has to be at least $q$. For the Hofstadter problem on a triangular lattice we find that there are exactly $q$ minima located at the wavevectors $(0,4 \pi \ell p / q)$ with $\ell=0 \ldots q-1$. Analogously to the case of the square lattice in Section IIB, we can now proceed and find for the transformation laws of the 
vortex fields $\varphi_{\ell}$ at these minima

$$
\begin{aligned}
T_{1} & : \varphi_{\ell} \rightarrow \varphi_{\ell+1} \\
T_{2} & : \varphi_{\ell} \rightarrow \varphi_{\ell} \omega^{-2 \ell} \\
T_{d} & : \varphi_{\ell} \rightarrow \varphi_{\ell+1} \omega^{-(2 \ell+1)} \\
R_{\pi / 3}^{\text {dual }} & : \varphi_{\ell} \rightarrow \frac{e^{-i \pi(q-1) / 12}}{\sqrt{q}} \sum_{m=0}^{q-1} \varphi_{m} \omega^{(\ell-2 m) \ell} .
\end{aligned}
$$

It can be verified that these transformation laws obey Eqs. (B5) and (B6).

$$
\text { 2. } q=4 \nu
$$

If $q$ is a multiple of 4 we only have $q / 2$ different vortex minima which are located at the wavevectors $(0,4 \pi \ell p / q)$ with $\ell=0 \ldots q / 2-1$. The transformations among these fields are

$$
\begin{aligned}
T_{1}: \varphi_{\ell} & \rightarrow \varphi_{\ell+1} \\
T_{2}: \varphi_{\ell} & \rightarrow \varphi_{\ell} \omega^{-2 \ell} \\
T_{d}: \varphi_{\ell} & \rightarrow \varphi_{\ell+1} \omega^{-(2 \ell+1)} \\
R_{\pi / 3}^{\text {dual }} & : \varphi_{\ell} \rightarrow \frac{e^{-i p \pi / 12}}{\sqrt{q / 2}} \sum_{m=0}^{q / 2-1} \varphi_{m} \omega^{(\ell-2 m) \ell} .
\end{aligned}
$$

These also obey Eqs. (B5) and (B6).

\section{APPENDIX C: VORTEX BAND STRUCTURE AND PSG IN THE "SYMMETRIC GAUGE"}

In this appendix we show how to directly obtain a permutative representation of the PSG for $f=1 / 4$ by a judicious choice of gauge for the dual magnetic flux. We will loosely call this the "symmetric gauge". It is most conveniently expressed pictorially and is shown in Fig. 16, along with the corresponding magnetic unit cell labelling convention. A unique property of this gauge choice, that will play a crucial role in the discussion below, is that the Bravais net of the magnetic lattice is invariant under $\pi / 2$ rotations. It is clear that such a gauge choice is not possible in general, but is at least for filling factor $f=p / q$, satisfying the condition $q=2, n^{2}, n=2,3,4, \ldots$ (for the square lattice). We do not, however, explore this beyond $q=4$.

Directly diagonalizing the lattice vortex action in Eq. (2.8) we find 4 low energy vortex modes at wavevectors:

$$
\begin{aligned}
& \mathbf{k}_{0}=(0,-\pi / 2), \\
& \mathbf{k}_{1}=(\pi,-\pi / 2), \\
& \mathbf{k}_{2}=(\pi, \pi / 2), \\
& \mathbf{k}_{3}=(0, \pi / 2),
\end{aligned}
$$

which are defined in the first Brillouin zone of the magnetic lattice and we have taken the magnetic lattice constant to be equal to one. The corresponding normalized

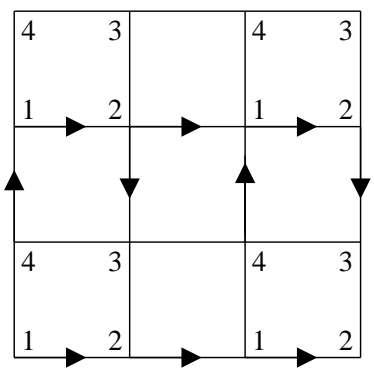

FIG. 16: Pictorial representation of the "symmetric gauge" . Arrows represent a factor $e^{-i \pi / 2}$. Magnetic unit cell labelling convention is also shown.

eigenvectors are given by:

$$
\begin{aligned}
& v^{0}=(0,1 / 2,1 / \sqrt{2}, 1 / 2), \\
& v^{1}=(-i / 2,1 / \sqrt{2}, 1 / 2,0), \\
& v^{2}=(1 / \sqrt{2}, i / 2,0,1 / 2), \\
& v^{3}=(1 / 2,0,1 / 2,1 / \sqrt{2}),
\end{aligned}
$$

As before, concentrating on the long-wavelength vortex dynamics near the transition, the vortex fields can be written as linear combinations of the 4 low-energy vortex modes:

$$
\psi_{\alpha}(\mathbf{R})=\sum_{\ell=0}^{3} v_{\alpha}^{\ell}(\mathbf{R}) \zeta_{\ell},
$$

where $\mathbf{R}$ labels magnetic unit cells, $\alpha$ labels sites within each magnetic unit cell and

$$
v_{\alpha}^{\ell}(\mathbf{R})=v_{\alpha}^{\ell} e^{i \mathbf{k}_{\ell} \cdot \mathbf{R}} .
$$

Using Eqs.(2.8) and (C3) we can work out the transformation properties of the low-energy vortex modes $\varphi_{\ell}$ under the symmetry operations of the microscopic Hamiltonian (2.2). These transformations turn out to have the following form:

$$
\begin{aligned}
& T_{x}: \zeta_{0} \rightarrow \zeta_{3}, \zeta_{1} \rightarrow \zeta_{2}, \zeta_{2} \rightarrow-\zeta_{1}, \zeta_{3} \rightarrow \zeta_{0}, \\
& T_{y}: \zeta_{0} \rightarrow \zeta_{1}, \zeta_{1} \rightarrow \zeta_{0}, \zeta_{2} \rightarrow i \zeta_{3}, \zeta_{3} \rightarrow i \zeta_{2}, \\
& R_{\pi / 2}^{\text {dir }}: \zeta_{0} \rightarrow \zeta_{1}, \zeta_{1} \rightarrow i \zeta_{2}, \zeta_{2} \rightarrow \zeta_{3}, \zeta_{3} \rightarrow \zeta_{0}, \\
& R_{\pi / 2}^{\text {dual }}: \zeta_{0} \rightarrow \zeta_{0}, \zeta_{1} \rightarrow-\zeta_{3}, \zeta_{2} \rightarrow i \zeta_{2}, \zeta_{3} \rightarrow \zeta_{1}, \\
& I_{x}^{\text {dir }}: \zeta_{0} \rightarrow i \zeta_{1}^{*}, \zeta_{1} \rightarrow i \zeta_{0}^{*}, \zeta_{2} \rightarrow \zeta_{3}^{*}, \zeta_{3} \rightarrow \zeta_{2}^{*}, \\
& I_{y}^{\text {dir }}: \zeta_{0} \rightarrow \zeta_{3}^{*}, \zeta_{1} \rightarrow \zeta_{2}^{*}, \zeta_{2} \rightarrow \zeta_{1}^{*}, \zeta_{3} \rightarrow \zeta_{0}^{*}, \\
& I_{x}^{\text {dual }}: \zeta_{0} \rightarrow-\zeta_{0}^{*}, \zeta_{1} \rightarrow-\zeta_{1}^{*}, \zeta_{2} \rightarrow \zeta_{2}^{*}, \zeta_{3} \rightarrow \zeta_{3}^{*}, \\
& I_{y}^{\text {dual }}: \zeta_{0} \rightarrow \zeta_{0}^{*}, \zeta_{1} \rightarrow-\zeta_{1}^{*}, \zeta_{2} \rightarrow \zeta_{2}^{*}, \zeta_{3} \rightarrow \zeta_{3}^{*} .
\end{aligned}
$$

Here $R_{\pi / 2}^{\text {dir }}$ denotes a $\pi / 2$-rotation about a direct lattice site, $R_{\pi / 2}^{\text {dual }}$ is a $\pi / 2$-rotation about a dual lattice site, $I_{x, y}^{\text {dir }}$ 
are reflections with respect to $x, y$ axes passing through a direct lattice site, while $I_{x, y}^{\text {dual }}$ are reflections with respect to $x, y$ axes passing through a dual lattice site. Clearly, the above rotational and translational transformations are all of the form of a unitary diagonal matrix multiplying a permutation matrix. Hence by composing each with a suitable global (gauge) phase rotation, they realize a permutative representation of the PSG.

It is straightforward to see that the most general quartic potential invariant under these transformations has the same form as Eq. (2.36). Thus one directly obtains in this manner the end result obtained in the text by first using Landau gauge and then unitarily transforming to $\zeta_{\ell}$ variables.

\section{APPENDIX D: SOME GROUND STATES AND THEIR PROPERTIES FOR $q=4$}

In this appendix we present a few more details of the structure of the saddle points of effective potential in Eq. (3.34) for the equal amplitude state identified as E in Fig 5. Writing $\zeta_{\ell} \propto e^{i \vartheta_{\ell}}$, the relevant term in the energy density (Euclidean Lagrange density) is (assuming first that $\lambda<0)$ :

$$
\begin{aligned}
\mathcal{L}_{4} & =-|\lambda|\left[\cos 2\left(\vartheta_{0}-\vartheta_{1}\right)-\cos 2\left(\vartheta_{1}-\vartheta_{2}\right)\right. \\
& \left.+\cos 2\left(\vartheta_{2}-\vartheta_{3}\right)+\cos 2\left(\vartheta_{3}-\vartheta_{0}\right)\right] .
\end{aligned}
$$

In these variables it is not immediately obvious what the ground state is. We change variables to

$$
\begin{aligned}
& \vartheta_{0}=\tilde{\vartheta}_{0}, \\
& \vartheta_{1}=\tilde{\vartheta}_{1}+\frac{\pi}{8}, \\
& \vartheta_{2}=\tilde{\vartheta}_{2}-\frac{\pi}{4}, \\
& \vartheta_{3}=\tilde{\vartheta}_{3}-\frac{\pi}{8} .
\end{aligned}
$$

In the new variables the mean-field energy density becomes:

$$
\begin{aligned}
\mathcal{L}_{4} & =-|\lambda|\left[\cos 2\left(\tilde{\vartheta}_{0}-\tilde{\vartheta}_{1}-\frac{\pi}{8}\right)+\cos 2\left(\tilde{\vartheta}_{1}-\tilde{\vartheta}_{2}-\frac{\pi}{8}\right)\right. \\
& \left.+\cos 2\left(\tilde{\vartheta}_{2}-\tilde{\vartheta}_{3}-\frac{\pi}{8}\right)+\cos 2\left(\tilde{\vartheta}_{3}-\tilde{\vartheta}_{0}-\frac{\pi}{8}\right)\right] .
\end{aligned}
$$

It is now clear that the minimum is achieved when

$$
\begin{aligned}
& \tilde{\vartheta}_{0}-\tilde{\vartheta}_{1}=0, \pi, \\
& \tilde{\vartheta}_{1}-\tilde{\vartheta}_{2}=0, \pi, \\
& \tilde{\vartheta}_{2}-\tilde{\vartheta}_{3}=0, \pi,
\end{aligned}
$$

and

$$
\begin{aligned}
& \tilde{\vartheta}_{0}-\tilde{\vartheta}_{1}=\frac{\pi}{4}, \frac{5 \pi}{4}, \\
& \tilde{\vartheta}_{1}-\tilde{\vartheta}_{2}=\frac{\pi}{4}, \frac{5 \pi}{4}, \\
& \tilde{\vartheta}_{2}-\tilde{\vartheta}_{3}=\frac{\pi}{4}, \frac{5 \pi}{4} .
\end{aligned}
$$

Thus the ground state has a 16-fold degeneracy. To see what do these states look like in real space it is sufficient to understand how they transform under the symmetry operations of the microscopic Hamiltonian Eq. (2.2). Using Eq. (C5) one finds that the ground state manifold has the following properties.

1. All 16 states are invariant under $T_{x}^{4}$ and $T_{y}^{4}$, i.e. the charge density pattern in the ground state has a period of 4 lattice constants in both $x$ - and $y$ directions.

2. There are 2 states that are invariant under rotations by $\pi / 2$ about a given direct lattice site. These states are, in the original variables:

$$
\begin{aligned}
& \vartheta_{0}-\vartheta_{1}=-\frac{\pi}{8}, \vartheta_{1}-\vartheta_{2}=\frac{3 \pi}{8}, \vartheta_{2}-\vartheta_{3}=-\frac{\pi}{8} \\
& \text { and } \\
& \vartheta_{0}-\vartheta_{1}=\frac{7 \pi}{8}, \vartheta_{1}-\vartheta_{2}=-\frac{5 \pi}{8}, \vartheta_{2}-\vartheta_{3}=\frac{7 \pi}{8}
\end{aligned}
$$

These 2 states are related to each other by $\left(T_{x} T_{y}\right)^{2}$ transformation. More simply, this implies that each state has two inequivalent centers of rotation within each unit cell.

3. The states are also invariant under $I_{x}$ and $I_{y}$ transformations through the centers of rotation.

4. All 16 states are related to each other by successive $T_{x}$ or $T_{y}$ operations.

It is then clear that the ground state is a charge density wave (CDW), of the general form shown in Fig.13.

Now consider the case $\lambda>0$. In this case there are 16 degenerate ground states as well. They are given by:

$$
\begin{aligned}
& \tilde{\vartheta}_{0}-\tilde{\vartheta}_{1}=\frac{\pi}{2}, \frac{3 \pi}{2}, \\
& \tilde{\vartheta}_{1}-\tilde{\vartheta}_{2}=\frac{\pi}{2}, \frac{3 \pi}{2}, \\
& \tilde{\vartheta}_{2}-\tilde{\vartheta}_{3}=\frac{\pi}{2}, \frac{3 \pi}{2} .
\end{aligned}
$$

and

$$
\begin{aligned}
& \tilde{\vartheta}_{0}-\tilde{\vartheta}_{1}=-\frac{\pi}{4}, \frac{3 \pi}{4}, \\
& \tilde{\vartheta}_{1}-\tilde{\vartheta}_{2}=-\frac{\pi}{4}, \frac{3 \pi}{4}, \\
& \tilde{\vartheta}_{2}-\tilde{\vartheta}_{3}=-\frac{\pi}{4}, \frac{3 \pi}{4} .
\end{aligned}
$$

These states have exactly the same symmetry properties as the ones at $\lambda<0$ and thus are physically the same states. The two ground states that are invariant under $R_{\frac{\pi}{2}}$ in this case are:

$$
\vartheta_{0}-\vartheta_{1}=\frac{3 \pi}{8}, \vartheta_{1}-\vartheta_{2}=\frac{7 \pi}{8}, \vartheta_{2}-\vartheta_{3}=\frac{3 \pi}{8},
$$

and

$$
\vartheta_{0}-\vartheta_{1}=-\frac{5 \pi}{8}, \vartheta_{1}-\vartheta_{2}=-\frac{\pi}{8}, \vartheta_{2}-\vartheta_{3}=-\frac{5 \pi}{8}
$$




\section{APPENDIX E: PERMUTATIVE REPRESENTATIONS OF THE PSG}

As discussed in Sec. III A, the problem of determining whether a fractionalized boson interpretation of the $q$ vortex theory at filling $f=p / q$ reduces to a problem in representation theory. In particular, we must find $q$ dimensional matrices $x, y, r$ representing the generators $T_{x}, T_{y}, R_{\pi / 2}^{\text {dual }}$ (for this appendix we neglect the inversion operations)

$$
\begin{aligned}
x & =\bar{x} \hat{x}, \\
y & =\bar{y} \hat{y}, \\
r & =\bar{r} \hat{r},
\end{aligned}
$$

where $\bar{x}, \bar{y}, \bar{r}$ are diagonal unitary matrices (i.e. diagonal matrices with all entries of absolute value one), and $\hat{x}, \hat{y}, \hat{z}$ are permutation matrices. We seek matrices of this form satisfying

$$
\begin{aligned}
x y & =\omega y x, \\
r^{-1} x r & =y^{-1}, \\
r^{-1} y r & =x, \\
r^{4} & =1,
\end{aligned}
$$

with $\omega^{k}=1$ if and only if $k=0(\bmod \mathrm{q})$. The form of Eq. (E1) requires that the permutation (hat) parts of the generators satisfy very similar relations to Eq. (E2), to wit:

$$
\begin{aligned}
\hat{x} \hat{y} & =\hat{y} \hat{x}, \\
\hat{r}^{-1} \hat{x} \hat{r} & =\hat{y}^{-1}, \\
\hat{r}^{-1} \hat{y} \hat{r} & =\hat{x}, \\
\hat{r}^{4} & =1 .
\end{aligned}
$$

This leads to a few general observations. First, recall that any permutation can be written (decomposed) as a product of disjoint cycles. Since $\hat{r}^{4}=1, \hat{r}$ can contain only cycles of length 1,2 , and 4 . Hence, $\hat{r}^{2}$ contains only 1 -cycles and 2-cycles, and an even number of the latter. Another interesting identity is

$$
r^{2} y r^{2}=r^{-2} y r^{2}=r^{-1} x r=y^{-1}
$$

Hence

$$
\begin{aligned}
& r^{2} y r^{2} y=1, \\
& r^{2} x r^{2} x=1
\end{aligned}
$$

the latter equation being obtained similarly. Thus $\hat{r}^{2} \hat{x}$ can contain only 1-cycles and 2-cycles as well.

Finally, to obtain a non-vanishing commutator of $x$ and $y$, clearly at least one of $\hat{x}, \hat{y}$ must not be the identity permutation.

Unfortunately we have so far been unable to solve this representation problem generally. The explicit examples for $q=2,4$ have been discussed in the text. In addition, we have been able to prove there is no such representation for $q=3$. This proof follows. First, note that $\hat{r}$ can contain either one 2-cycle or be trivial. Suppose first $\hat{r}=1$. Then from the third of Eqs. (E3), we see that $\hat{x}=\hat{y}=\hat{p}$, some permutation which must be non-trivial according to the above consideration. But since $\hat{r}=1$, the second of Eqs. (E3) implies $\hat{p}^{2}=1$. Thus, without loss of generality, we can take $\hat{p}=(12)$ (we use cycle notation, so this is just the permutation of the first two objects). Inserting this into $x y=\omega y x$, one finds that, since the third element is not permuted, $\bar{x}_{33} \bar{y}_{33}=\omega \bar{x}_{33} \bar{y}_{33}$, which is impossible. Thus our assumption that $\hat{r}=1$ must be false.

Hence if such a representation exists, we must take $\hat{r}$ to be a 2-cycle, and without loss of generality, we may take $\hat{r}=(12)$. Thus $\hat{r}^{2}=1$, so from Eq. (E5), $\hat{x}^{2}=1$ and $\hat{y}^{2}=1$. Now at least one of $\hat{x}, \hat{y}$ is non-trivial, so let us assume, again without loss of generality, that $\hat{x} \neq 1$. It must be a 2-cycle since its square is unity. There are only two inequivalent possibilities. If $\hat{x}=(12)$, then $\hat{x}=\hat{r}$, which implies $\hat{y}=(12)$ as well. We have already seen that there is no solution with $\hat{x}=\hat{y}=(12)$, so this is inconsistent. The other inequivalent choice is $\hat{x}=(23)$. Then we can compute $\hat{y}=\hat{r} \hat{x} \hat{r}^{-1}=(13)$. But it is easy to see that this violates the first of Eqs. (E3).

Thus all assumptions for $q=3$ have been proven inconsistent save one: that no such representation exists.

We have found some explicit examples for $q=8,9$.

For $q=8$, with the notation $\gamma=e^{i \pi / 16}\left(\gamma^{4}=\omega\right)$, we find

$$
\begin{aligned}
& x=\left(\begin{array}{cccccccc}
0 & 0 & 0 & 0 & 0 & \gamma^{9} & 0 & 0 \\
\gamma^{30} & 0 & 0 & 0 & 0 & 0 & 0 & 0 \\
0 & 0 & 0 & 0 & 0 & 0 & 0 & \gamma^{15} \\
0 & 0 & \gamma^{2} & 0 & 0 & 0 & 0 & 0 \\
0 & \gamma^{17} & 0 & 0 & 0 & 0 & 0 & 0 \\
0 & 0 & 0 & 0 & 1 & 0 & 0 & 0 \\
0 & 0 & 0 & \gamma^{23} & 0 & 0 & 0 & 0 \\
0 & 0 & 0 & 0 & 0 & 0 & 1 & 0
\end{array}\right), \\
& y=\left(\begin{array}{cccccccc}
0 & 0 & 0 & 0 & 0 & 0 & \gamma^{23} & 0 \\
0 & 0 & 0 & 0 & 0 & 0 & 0 & \gamma^{17} \\
1 & 0 & 0 & 0 & 0 & 0 & 0 & 0 \\
0 & 1 & 0 & 0 & 0 & 0 & 0 & 0 \\
0 & 0 & \gamma^{15} & 0 & 0 & 0 & 0 & 0 \\
0 & 0 & 0 & \gamma^{9} & 0 & 0 & 0 & 0 \\
0 & 0 & 0 & 0 & \gamma^{2} & 0 & 0 & 0 \\
0 & 0 & 0 & 0 & 0 & \gamma^{30} & 0 & 0
\end{array}\right), \\
&\left(\begin{array}{cccccccc}
0 & 0 & 0 & 0 & 0 & 0 & 1 & 0 \\
0 & 0 & 0 & 0 & 1 & 0 & 0 & 0 \\
0 & 0 & 0 & 0 & 0 & 0 & 0 & 1 \\
0 & 0 & 0 & 0 & 0 & 1 & 0 & 0 \\
0 & 0 & 1 & 0 & 0 & 0 & 0 & 0 \\
1 & 0 & 0 & 0 & 0 & 0 & 0 & 0 \\
0 & 0 & 0 & 1 & 0 & 0 & 0 & 0 \\
0 & 1 & 0 & 0 & 0 & 0 & 0 & 0
\end{array}\right) .
\end{aligned}
$$

For $q=9$, with the notation $\tilde{\omega}=e^{i \pi / 9}\left(\tilde{\omega}^{2}=\omega\right)$, we 
find

$$
\begin{aligned}
& x=\left(\begin{array}{ccccccccc}
0 & 0 & \tilde{\omega}^{5} & 0 & 0 & 0 & 0 & 0 & 0 \\
1 & 0 & 0 & 0 & 0 & 0 & 0 & 0 & 0 \\
0 & \tilde{\omega}^{16} & 0 & 0 & 0 & 0 & 0 & 0 & 0 \\
0 & 0 & 0 & 0 & 0 & \tilde{\omega}^{9} & 0 & 0 & 0 \\
0 & 0 & 0 & 1 & 0 & 0 & 0 & 0 & 0 \\
0 & 0 & 0 & 0 & 1 & 0 & 0 & 0 & 0 \\
0 & 0 & 0 & 0 & 0 & 0 & 0 & 0 & \tilde{\omega}^{13} \\
0 & 0 & 0 & 0 & 0 & 0 & \tilde{\omega}^{2} & 0 & 0 \\
0 & 0 & 0 & 0 & 0 & 0 & 0 & 1 & 0
\end{array}\right), \\
& y=\left(\begin{array}{ccccccccc}
0 & 0 & 0 & 0 & 0 & 0 & \tilde{\omega}^{13} & 0 & 0 \\
0 & 0 & 0 & 0 & 0 & 0 & 0 & \tilde{\omega}^{9} & 0 \\
0 & 0 & 0 & 0 & 0 & 0 & 0 & 0 & \tilde{\omega}^{5} \\
\tilde{\omega}^{2} & 0 & 0 & 0 & 0 & 0 & 0 & 0 & 0 \\
0 & 1 & 0 & 0 & 0 & 0 & 0 & 0 & 0 \\
0 & 0 & 1 & 0 & 0 & 0 & 0 & 0 & 0 \\
0 & 0 & 0 & 1 & 0 & 0 & 0 & 0 & 0 \\
0 & 0 & 0 & 0 & 1 & 0 & 0 & 0 & 0 \\
0 & 0 & 0 & 0 & 0 & \tilde{\omega}^{16} & 0 & 0 & 0
\end{array}\right) \text {, } \\
& r=\left(\begin{array}{lllllllll}
0 & 0 & 0 & 0 & 0 & 0 & 1 & 0 & 0 \\
0 & 0 & 0 & 1 & 0 & 0 & 0 & 0 & 0 \\
1 & 0 & 0 & 0 & 0 & 0 & 0 & 0 & 0 \\
0 & 0 & 0 & 0 & 0 & 0 & 0 & 1 & 0 \\
0 & 0 & 0 & 0 & 1 & 0 & 0 & 0 & 0 \\
0 & 1 & 0 & 0 & 0 & 0 & 0 & 0 & 0 \\
0 & 0 & 0 & 0 & 0 & 0 & 0 & 0 & 1 \\
0 & 0 & 0 & 0 & 0 & 1 & 0 & 0 & 0 \\
0 & 0 & 1 & 0 & 0 & 0 & 0 & 0 & 0
\end{array}\right)
\end{aligned}
$$

These were obtained as follows. First, we obtained $\hat{x}, \hat{y}, \hat{r}$ from a simple physical construction. In particular, we constructed a covering of the sites of the dual square lattice by $q$ sublattices. For $q=n^{2}$ ( $n=3$ for $q=9$ above), these are chosen by breaking the lattice up into a square grid of $n \times n$ blocks, labeling the sites in each block identically from $\ell=1 \ldots q$. Taking the site of the direct lattice at the center of one of these blocks as the origin, one can see that rotations and translations all act as permutations of the $q$ labels. These permutations are taken as $\hat{x}, \hat{y}, \hat{r}$ and by construction satisfy Eqs. (E3). For $q=2 n^{2}$ ( $n=2$ for $q=8$ above), we break the sites of the dual lattice again up into a square grid of $n \times n$ blocks, and further break up these blocks into staggered $A$ and B sublattices. We label the sites of each block of the A sublattice identically from $1 \ldots n^{2}$ and the sites of each block of the B sublattice identically from $n^{2}+1 \ldots 2 n^{2}$. Now taking the origin as a point of the direct lattice at the corner of two A and two B blocks, we once again obtain from physical rotations a set of permutations on $q$ variables, which solve Eqs. (E3).

We then take $\bar{x}, \bar{y}, \bar{r}$ as unknowns, and look for solutions of Eqs. (E2). These reduce to many redundant equations for $3 q$ unknowns (the phases of each diagonal entry of $\bar{x}, \bar{y}, \bar{r})$. We find for $q=8,9$ many solutions, of which the above examples are particular ones. This procedure can clearly be generalized to arbitrary $n$ as defined above. We do not, however, have a proof at present that solutions for the diagonal unknowns exist in general. The overabundance of solutions for $q=8,9$ suggests, however, that they do exist - hence the speculation in the text.

\section{APPENDIX F: DUALITY FOR $q$-SLAVE BOSON GAUGE THEORY}

In this appendix, we directly dualize the hamiltonian $\mathcal{H}_{\tilde{q}}=\mathcal{H}_{\tilde{q}}^{0}+\mathcal{H}_{\tilde{q}}^{1}$ in Eqs. (3.13), (3.15). The first step is to shift the slave rotor number operators to new variables with an average zero density:

$$
\hat{n}_{i \ell} \rightarrow \hat{n}_{i \ell}+p m_{i \ell}
$$

Note that, using $\tilde{q}=q$, the spatial average of $p m_{i \ell}$ is $p / q=f$, so that the shifted $\hat{n}_{i \ell}$ fields have zero spatial average. This shift is allowed and canonical because $p$ is an integer. After this shift, the Hamiltonian becomes, up to a constant,

$$
\begin{aligned}
& \mathcal{H}_{\tilde{q}}= \\
& -t \sum_{i \ell} \cos \left(\Delta_{\alpha} \hat{\phi}_{i \ell}-\mathcal{A}_{i \alpha}^{\ell}+\mathcal{A}_{i \alpha}^{\ell-1}\right) \\
& +u\left(\hat{n}_{i \ell}-\tilde{\mu}\left(m_{i \ell}-\frac{1}{q}\right)\right)^{2} \\
& +\frac{v}{2} \sum_{i \ell}\left|\overrightarrow{\mathcal{E}}_{i}^{\ell}\right|^{2}-K \sum_{a \ell} " \cos ^{" \prime}\left(\vec{\Delta} \times \overrightarrow{\mathcal{A}}^{\ell}\right)_{a}
\end{aligned}
$$

with $\tilde{\mu}=-\left(\tilde{\mu}_{s}-p\right) / 2$. The Gauss' law constraint is changed to

$$
\left[\vec{\Delta} \cdot \overrightarrow{\mathcal{E}}^{\ell}\right]_{i}=\hat{n}_{i, \ell}-\hat{n}_{i, \ell+1}+\epsilon_{i \ell}
$$

At this point, we can recover the analysis of Sec. III B 2 by assuming the bosons are gapped, $\hat{n}_{i \ell} \approx 0$, and treating the pure compact $U(1)^{q-1}$ gauge theory with the static source charges $\epsilon_{i \ell}$. Here, we will connect to the dual $q$-vortex theory directly. This is accomplished by performing a separate duality transformation for each of the $q$ fractional boson fields. This is conveniently done, as elsewhere in the paper, in a path integral formulation. The appropriate world-line form of the partition function, analogous to Eq. (2.5), but with the gauge fields included is

$\mathcal{Z}=\sum_{\left\{J^{\ell}, \mathcal{B}^{\ell}\right\}} \int d \mathcal{A}^{\ell} \prod_{i \ell} \delta\left(\Delta \cdot J_{i}^{\ell}\right) \prod_{a \mu} \delta\left(\sum_{\ell} \mathcal{B}_{\mu}^{\ell}\right) e^{-\mathcal{S}_{q}},(\mathrm{~F}$

where

$$
\begin{aligned}
\mathcal{S}_{q}= & \sum_{i \ell} \frac{1}{2 e^{2}}\left|J_{i}^{\ell}-\tilde{\mu}\left(m_{i \ell}-\frac{1}{q}\right) \hat{\tau}\right|^{2} \\
& -i\left(J_{i}^{\ell}-J_{i}^{\ell+1}+\epsilon_{i \ell} \hat{\tau}\right) \cdot \mathcal{A}_{i}^{\ell} \\
+ & \sum_{a \ell} i \frac{\mathcal{B}_{a}^{\ell}}{2 \pi} \cdot\left(\Delta \times \mathcal{A}^{\ell}\right)_{a}+\frac{\tilde{e}^{2}}{2}\left|\mathcal{B}_{a}^{\ell}\right|^{2} .
\end{aligned}
$$


Here and in the remainder of this section, we will suppress 3 -vector indices and spatial coordinate labels when possible, and use the usual vector notations $(\cdot, \times)$ for 3 -vector operations. Physically, $J^{\ell}=\left(n_{\ell}, \overrightarrow{J_{\ell}}\right)$ is an integer-valued vector field representing the 3 -current of fractional boson $\ell, \mathcal{A}^{\ell}=\left(\mathcal{A}_{0}^{\ell}, \overrightarrow{\mathcal{A}}^{\ell}\right)$ is the $2 \pi$-periodic 3 -vector potential for the $\ell^{t h}$ gauge symmetry. The $\mathcal{B}^{\ell}$ is a $2 \pi \times$ integer-valued field defined on the links of the dual lattice, which, when summed over, gives a Villain potential for the $\ell^{\text {th }}$ gaugefield kinetic energy. Finally, $\hat{\tau}=(1,0,0)$ is the unit vector in the time direction.

To proceed, we solve the divergence constraints on the boson currents by defining vector potentials $A_{a}^{\ell}$ on the dual lattice,

$$
J_{i}^{\ell}=\frac{\left(\Delta \times A^{\ell}\right)_{i}}{2 \pi},
$$

and for convenience we find some fixed non-fluctuating $2 \pi \times$ integer fields $\overline{\mathcal{B}}^{\ell}$ on the dual lattice satisfying

$$
\frac{\left(\Delta \times \overline{\mathcal{B}}^{\ell}\right)_{i}}{2 \pi}=\epsilon_{i \ell} \hat{\tau} .
$$

Since $\sum_{\ell} \epsilon_{i \ell}=0$, the background fields can (and should!) be chosen to satisfy $\sum_{\ell} \bar{B}^{\ell}=0$. Inserting these into the partition function, one has

$$
\mathcal{Z}=\sum_{\left\{A^{\ell}, \mathcal{B}^{\ell}\right\}} \int d \mathcal{A}^{\ell} \prod_{a \mu} \delta\left(\sum_{\ell} \mathcal{B}_{\mu}^{\ell}\right) e^{-\mathcal{S}_{q}^{1}},
$$

with

$$
\begin{aligned}
\mathcal{S}_{q}^{1}= & \sum_{i \ell} \frac{1}{8 \pi^{2} e^{2}}\left|\left(\Delta \times A^{\ell}\right)_{i}-2 \pi \tilde{\mu}\left(m_{i \ell}-\frac{1}{q}\right) \hat{\tau}\right|^{2} \\
& -\frac{i}{2 \pi} \mathcal{A}_{i}^{\ell} \cdot\left[\Delta \times\left(A^{\ell}-A^{\ell+1}+\overline{\mathcal{B}}^{\ell}-\mathcal{B}^{\ell}\right)\right]_{i} \\
+ & \sum_{a \ell} \frac{\tilde{e}^{2}}{2}\left|\mathcal{B}_{a}^{\ell}\right|^{2} .
\end{aligned}
$$

At this point one may integrate out the $\mathcal{A}^{\ell}$ fields, which gives a delta-function constraining

$$
\Delta \times \mathcal{B}^{\ell}=\Delta \times\left(A^{\ell}-A^{\ell+1}+\bar{B}^{\ell}\right) .
$$

The general solution is

$$
\mathcal{B}_{\mu}^{\ell}=A_{\mu}^{\ell}-A_{\mu}^{\ell+1}+\bar{B}_{\mu}^{\ell}-\Delta_{\mu} \chi_{\ell},
$$

where $\chi_{\ell}$ is a $2 \pi \times$ integer-valued scalar field. To preserve the constraint $\sum_{\ell} \mathcal{B}^{\ell}=0$, we should impose $\sum_{\ell} \chi_{\ell}=0$ (or at least a constant). The partition function becomes

$$
\mathcal{Z}=\sum_{\left\{A^{\ell}, \chi_{\ell}\right\}} \prod_{a} \delta\left(\sum_{\ell} \chi_{a \ell}\right) e^{-\mathcal{S}_{q}^{2}}
$$

with

$$
\begin{aligned}
\mathcal{S}_{q}^{2}= & \sum_{i \ell} \frac{1}{8 \pi^{2} e^{2}}\left|\left(\Delta \times A^{\ell}\right)_{i}-2 \pi \tilde{\mu}\left(m_{i \ell}-\frac{1}{q}\right) \hat{\tau}\right|^{2} \\
& +\sum_{a \ell} \frac{\tilde{e}^{2}}{2}\left|A_{\mu}^{\ell}-A_{\mu}^{\ell+1}+\overline{\mathcal{B}}_{\mu}^{\ell}-\Delta_{\mu} \chi_{\ell}\right|^{2} .
\end{aligned}
$$

Next we "soften" the $2 \pi \times$ integer constraints on $\chi_{\ell}, A^{\ell}$, converting the partition sum to an integral, and adding terms in the action to weight integer values more heavily (alternatively, this can be done exactly by Poisson resummation, but we prefer to keep things simple):

$$
\mathcal{Z}=\int d A^{\ell} d \chi_{\ell} \prod_{a} \delta\left(\sum_{\ell} \chi_{a \ell}\right) e^{-\mathcal{S}_{q}^{3}}
$$

with

$$
\mathcal{S}_{q}^{3}=\mathcal{S}_{q}^{2}-\sum_{a \ell}\left[y_{q v} \cos A_{a \mu}^{\ell}+\lambda \cos \chi_{a \ell}\right] .
$$

At this point it is advantageous to perform a few manipulations in a row to bring the partition function into a simple form. Rather than show the results of the individual steps, we simply give the procedure, to be executed in order:

1. Decompose the background fields into longitudinal and transverse parts according to $\overline{\mathcal{B}}_{\mu}^{\ell}=\Delta_{\mu} \eta_{\ell}+\breve{\mathcal{B}}_{\mu}^{\ell}$, where $\Delta \cdot \breve{\mathcal{B}}^{\ell}=0$, and one chooses $\sum_{\ell} \eta_{\ell}=0$.

2. Shift $\chi_{a \ell} \rightarrow \chi_{a \ell}-\vartheta_{a \ell}+\vartheta_{a, \ell+1}+\eta_{a \ell}$, and integrate the partition function over the $\vartheta_{a \ell}$ so introduced at every dual lattice site.

3. Next shift $\vartheta_{\ell} \rightarrow \vartheta_{\ell}+\sum_{\ell^{\prime}=\ell}^{q-1} \chi_{\ell^{\prime}}$.

4. Finally, shift $A_{\mu}^{\ell} \rightarrow A_{\mu}^{\ell}-\Delta_{\mu} \vartheta_{\ell}$.

At this point, all explicit $\chi_{\ell}$ dependence will be eliminated from the action, and the integral over $\chi_{\ell}$ can be done to give an unimportant constant multiplier. The rescaled partition function is then

$$
\mathcal{Z}=\int d A^{\ell} d \vartheta_{\ell} e^{-\mathcal{S}_{q}^{4}},
$$

with

$$
\begin{aligned}
& \mathcal{S}_{q}^{4}=\sum_{i \ell} \frac{1}{8 \pi^{2} e^{2}}\left|\left(\Delta \times A^{\ell}\right)_{i}-2 \pi \tilde{\mu}\left(m_{i \ell}-\frac{1}{q}\right) \hat{\tau}\right|^{2} \\
& +\sum_{a \ell} \frac{\tilde{e}^{2}}{2}\left|A_{\mu}^{\ell}-A_{\mu}^{\ell+1}+\breve{\mathcal{B}}_{\mu}^{\ell}\right|^{2} \\
& -\sum_{a \ell}\left[y_{q v} \cos \left(\Delta_{\mu} \vartheta_{\ell}-A_{\mu}^{\ell}\right)+\cos \left(\vartheta_{\ell}-\vartheta_{\ell+1}-\eta_{\ell}\right)\right] .
\end{aligned}
$$

We have nearly achieved the desired dual form. Indeed, due to the $\tilde{e}^{2}$ "Higgs" term, the only gapless gauge fluctuations are those with $A^{\ell}=A$ equal for all $\ell$. The relative fluctuations of the different $A^{\ell}$ fields will be small 
and can be neglected. There is in general, however, a mean value (average over configurations) for each of the $A^{\ell}$ fields, or more importantly the fluxes $\Delta \times A^{\ell}$. This mean value is oscillatory and has zero spatial average, since both the $m_{i \ell}-1 / q$ term has zero spatial average as does $\Delta \times \breve{\mathcal{B}}^{\ell}=2 \pi \epsilon_{i \ell} \hat{\tau}$. Once can show by a consideration of the vortex band structure that, generically, the uniform vortex modes are essentially unaffected by this spatially oscillating flux with zero average. A careful inspection shows, however, that the staggered potential $\mu_{s}$ is crucial here. For the non-generic case $\mu_{s}=0$, so that $\tilde{\mu}=p / 2$, one can show that the average flux is

$$
\left\langle\left(\Delta \times A^{\ell}\right)_{i}\right\rangle=2 \pi p\left(m_{i \ell}-\frac{1}{q}\right), \quad \text { for } \mu_{s}=0 .
$$

Although this has zero spatial average, this zero value is deceiving, since the background flux $\Delta \times A^{\ell}$ differs on different plaquettes by a multiple of $2 \pi$. Since the vortex kinetic energy is $2 \pi$-periodic, such a "zero average" flux has the effect of a uniform one, and drastically modifies the vortex spectrum. In the generic situation with $\mu_{s} \neq 0$, however, the differences in fluxes between plaquettes is truly non-trivial, and it is correct to neglect this oscillating average flux.

In this case, we may take the continuum limit by setting $A^{\ell} \approx A, e^{i \vartheta_{\ell}} \rightarrow|\bar{\varphi}|^{-1} \zeta_{\ell}$, adding potential terms that keep the magnitude of $\zeta_{\ell}$ approximately constant, and assuming slow spatial variations of the $\zeta_{\ell}$ fields. One obtains, after coarse-graining, an effective potential of the same form as Eq. (3.33) in the main text.
1 N. P. Ong, Y. Wang, S. Ono, Y. Ando, and S. Uchida, Annalen der Physik 13, 9 (2004); Y. Wang, S. Ono, Y. Onose, G. Gu, Y. Ando, Y. Tokura, S. Uchida, and N. P. Ong, Science 29986 (2003).

2 V. J. Emery and S. A. Kivelson, Nature 374, 434 (1995); N. Trivedi and M. Randeria, Phys. Rev. Lett. 75, 312 (1995); M. Franz and A. J. Millis, Phys. Rev. B 58, 14572 (1998); H.-J. Kwon and A. T. Dorsey, Phys. Rev. B 59, 6438 (1999).

3 M. A. Steiner, G. Boebinger, and A. Kapitulnik, condmat/0406232; M. A. Steiner and A. Kapitulnik, condmat/0406227.

4 S. A. Kivelson, E. Fradkin, V. Oganesyan, I. P. Bindloss, J. M. Tranquada, A. Kapitulnik, and C. Howald, Rev. Mod. Phys. 75, 1201 (2003).

5 A. Fang, C. Howald, N. Kaneko, M. Greven, and A. Kapitulnik, Phys. Rev. B 70, 214514 (2004).

6 M. Vershinin, S. Misra, S. Ono, Y. Abe, Y. Ando, and A. Yazdani, Science 303, 1995 (2004).

7 K. McElroy, D.-H. Lee, J. E. Hoffman, K. M. Lang, E. W. Hudson, H. Eisaki, S. Uchida, J. Lee, and J. C. Davis, cond-mat/0404005.

8 T. Hanaguri, C. Lupien, Y. Kohsaka, D.-H. Lee, M. Azuma, M. Takano, H. Takagi, and J. C. Davis, Nature 430, 1001 (2004).

${ }^{9}$ K.-S. Liu and M. E. Fisher, J. Low. Temp. Phys. 10, 655 (1972); J. M. Kosterlitz, D. R. Nelson, and M. E. Fisher Phys. Rev. B 13, 412 (1976).

10 S. Sachdev and E. Demler, Phys. Rev. B 69, 144504 (2004).

11 S.-C. Zhang, Science 275, 1089 (1997).

12 L. Balents, M. P. A. Fisher, and C. Nayak Phys. Rev. B 60, 1654 (1999); T. Senthil and M. P. A. Fisher Phys. Rev. B 62, 7850 (2000).

13 C. Lannert, M. P. A. Fisher, and T. Senthil, Phys. Rev. B 63, 134510 (2001).

14 S. Sachdev and K. Park, Annals of Physics, N.Y. 298, 58 (2002).

15 T. Senthil, A. Vishwanath, L. Balents, S. Sachdev, and M. P. A. Fisher, Science 303, 1490 (2004); T. Senthil, L. Balents, S. Sachdev, A. Vishwanath, and M. P. A. Fisher, Phys. Rev. B 70, 144407 (2004).
16 N. Read and S. Sachdev, Phys. Rev. Lett. 62, 1694 (1989); Phys. Rev. B 42, 4568 (1990).

17 E. Frey and L. Balents, Phys. Rev. B 55, 1050 (1997).

18 J. A. Hertz, Phys. Rev. B 14, 1165 (1976).

19 J. E. Hoffman, E. W. Hudson, K. M. Lang, V. Madhavan, S. H. Pan, H. Eisaki, S. Uchida, and J. C. Davis, Science 295, 466 (2002).

20 L. Balents, L. Bartosch, A. Burkov, S. Sachdev, and K. Sengupta, cond-mat/0409470; this companion paper is referred to as II in the text.

21 E. Fradkin and S. A. Kivelson, Mod. Phys. Lett. B 4, 225 (1990).

22 D. S. Rokhsar and S. A. Kivelson, Phys. Rev. Lett. 61, 2376 (1988).

23 S. Sachdev, Phys. Rev. B 40, 5204 (1989).

24 G. Baskaran and P. W. Anderson Phys. Rev. B 37, 580 (1988); S. Sachdev and N. Read, Int. J. Mod. Phys. B 5, 219 (1991), cond-mat/0402109; X.-G. Wen and P. A. Lee, Phys. Rev. Lett. 76, 503 (1996); Phys. Rev. Lett. 80, 2193 (1998).

25 C. Dasgupta and B. I. Halperin, Phys. Rev. Lett. 47, 1556 (1981).

26 D.R. Nelson, Phys. Rev. Lett. 60, 1973 (1988).

27 M. P. A. Fisher and D.-H. Lee, Phys. Rev. B 39, 2756 (1989); D.-H. Lee and M. P. A. Fisher, Phys. Rev. Lett. 63, 903 (1989).

28 J. Zak, Phys. Rev. 134, A1602 (1964); Phys. Rev. 134, A1607 (1964).

29 D. R. Hofstadter, Phys. Rev. B 14, 2239 (1976).

30 M. Hamermesh, Group Theory and its Application to Physical Problems, Dover, New York (1989), Chapter 12.

31 X.-G. Wen, Phys. Rev. B 65, 165113 (2002).

32 In fact, any of these operators can be composed with a $U(1)$ gauge transformation without any physical consequences. If we wish the gauge field to transform normally under the space-group operations of the PSG, this freedom reduces to the option to compose each of the generators with a global $U(1)$ rotation. This leaves Eq. (1.8) unchanged, while Eqs. (1.10) need only be satisfied up to an arbitrary phase factor. It is sometimes convenient to use this freedom to simplify explicit forms of the representation matrices. Of 
course, any such form can readily be transformed by phase rotations into a solution of Eqs. (1.10).

33 D. A. Ivanov, P. A. Lee, and X.-G. Wen, Phys. Rev. Lett. 84, 3958 (2000).

34 J. Zaanen, Z. Nussinov, S. I. Mukhin, Annals of Phys. 310, 181 (2004).

35 Z. Tešanović, Phys. Rev. Lett. 93, 217004 (2004); A. Melikyan and Z. Tešanović, cond-mat/0408344.

36 A. W. Sandvik, S. Daul, R. R. P. Singh, and D. J. Scalapino, Phys. Rev. Lett. 89, 247201 (2002).

37 S. Sachdev and M. Vojta, J. Phys. Soc. Jpn. 69, Suppl. B, 1 (2000), cond-mat/9910231.

38 B. I. Halperin, T. C. Lubensky, and S.-k. Ma, Phys. Rev. Lett. 32, 292 (1974).

39 J.-H. Chen, T. C. Lubensky, and D. R. Nelson, Phys. Rev. B 17, 4274 (1978).

40 E. Brézin, J. C. Le Guillou, and J. Zinn-Justin, Phys. Rev. B 10, 89 (1974).

41 S. Sachdev in Quantum magnetism, U. Schollwöck, J. Richter, D. J. J. Farnell, and R. A. Bishop eds, Lecture Notes in Physics, Springer, Berlin (2004); condmat/0401041.

42 J. Smiseth, E. Smørgrav, and A. Sudbø, Phys. Rev. Lett. 93, 077002 (2004).

43 N. Arkani-Hamed, A. G. Cohen, and H. Georgi, Phys. Rev. Lett. 86, 4757 (2001); C. T. Hill, S. Pokorski, and J. Wang, Phys. Rev. D 64, 105005 (2001).

44 O. Motrunich and A. Vishwanath, Phys. Rev. B 70, 075104 (2004).

45 M. Levin and T. Senthil, Phys. Rev. B 70, 220403 (2004).

46 Y. Zhang, E. Demler, and S. Sachdev, Phys. Rev. B 66, 094501 (2002).

47 A. Polkovnikov, M. Vojta, and S. Sachdev, Phys. Rev. B 65, 220509 (2002); A. Polkovnikov, S. Sachdev, and M. Vojta, Physica C 388-389, 19 (2003).

48 D. Podolsky, E. Demler, K. Damle, and B. I. Halperin, Phys. Rev. B, 67, 094514 (2003).

49 Y. Chen and C. S. Ting, Phys. Rev. B 65, 180513 (2002).

50 J.-X. Zhu, I. Martin, and A. R. Bishop, Phys. Rev. Lett. 89, 067003 (2002).

51 M. Franz, D. E. Sheehy, and Z. Tešanović, Phys. Rev. Lett. 88, 257005 (2002).

${ }^{52}$ H.-D. Chen, J.-P. Hu, S. Capponi, E. Arrigoni, and S.C. Zhang, Phys. Rev. Lett. 89, 137004 (2002).

53 A. Ghosal, C. Kallin, and A. J. Berlinsky, Phys. Rev. B 66, 214502 (2002).

54 B. M. Andersen, P. Hedegard, and H. Bruus, Phys. Rev. B 67, 134528 (2003).

${ }_{55}$ M. Takigawa, M. Ichioka, and K. Machida, J. Phys. Soc. Jpn. 73, 450 (2004).

${ }^{56}$ K. Park and S. Sachdev, Phys. Rev. B 64, 184510 (2001).

57 L. Bartosch, L. Balents, and S. Sachdev, condmat/0502002.

58 C. Wu, H.-D. Chen, J.-P. Hu, and S.-C. Zhang, Phys. Rev. A 69, 043609 (2004).

59 R. G. Melko, A. W. Sandvik, and D. J. Scalapino, Phys. Rev. B 69, 100408 (2004).

60 A. Kuklov, N. Prokof'ev, and B. Svistunov, Phys. Rev. Lett. 93, 230402 (2004).

61 Long range Coulomb interactions between Cooper pair bosons can be readily included, and do not significantly modify the present discussion.

62 The vortex degrees of freedom are defined only on the dual lattice sites, and the reader may wonder how we are able to obtain information on direct lattice sites in such a facile manner. The resolution is to note that Eq. (1.15) is a bilinear in the vortices. Therefore, by taking bilinear products of vortex operators on different sites around a single dual lattice plaquette, we can construct a measure of "density" on the direct lattice site on its center. We can similarly construct measures of bond variables such as the exchange or pairing energy. The advantage of the formalism in the paper is that all of this is automatically performed simply by studying the PSG properties of the observables, and then by evaluating Eq. (2.26) at the appropriate spatial position.

63 We caution that although such continuous transitions are allowed in principle, the actual critical theories in question are modified by fluctuation effects in $2+1$ dimensions. We know of no reliable analytic means of addressing the properties and stability of the strongly-coupled critical theories in question. Indeed, both $1 / N$ and $\epsilon=3-d$ expansion techniques are known to give incorrect results for the few examples of similar theories that can be addressed by other means (duality mappings and numerical simulation). These latter methods show that stable critical field theories exist for $q=1,2$, at least in a range of parameter space. Hence we expect that some but not all of the continuous transitions predicted at the mean field (in the $\varphi_{\ell}$ fields) level will remain generically continuous beyond mean field theory, while others may be driven first order by fluctuation effects. This is an important - and vast - open problem in critical phenomena which we will not address.

64 For the case of long-range Coulomb interactions with $\Lambda_{i j} \sim$ $1 /|i-j|$, the coupling $e^{2}$ will acquire a $\sim|k|$ momentum dependence. This will modify the renormalization group analysis in Section IIE, as discussed in M. P. A. Fisher and G. Grinstein, Phys. Rev. Lett. 60, 208 (1988) and J. Ye, Phys. Rev. B 58, 9450 (1998).

65 We were unable to find a similar result in the literature, though it or a more precise refinement of it likely exists. Therefore we give a brief argument here. For $f=1 / q$, the magnetic field is very small, and for the lowest energy states the continuum limit is a good zeroth order approximation. The lowest Hofstadter band should then be regarded as the lowest Landau level (LLL) of the continuum problem, weakly broadened by the periodic potential of the lattice. The states in the LLL correspond to electrons in Landau orbits that can be taken as Gaussian (e.g. in symmetric gauge) packets with a width of order the magnetic length, $\ell_{B} \sim \sqrt{q}$. The broadening of this LLL is due to the non-zero harmonics of the periodic potential, which oscillates on the lattice scale. Its matrix elements in the LLL are thus of order the Fourier transform of the aforementioned Gaussian packet at a reciprocal lattice vector $Q=(2 \pi, 2 \pi)$. Hence one expects the bandwidth $e^{- \text {const } Q^{2} \ell_{B}^{2}} \sim e^{-c q}$.

66 Note that other Mott states are possible for $p>1$, e.g. ones in which $p$ of the $q$ sites of the sublattice unit cell are occupied by one fractional boson, rather than one site being occupied by $p$ fractional bosons as assumed here for simplicity. Different such choices correspond to different PSGs.

67 The reader unfamiliar with $U(1)$ dualities may find it educational to compare this to the Villain version of duality carried out in the partition function, used elsewhere in the paper.

68 Of course, even in the deconfined scenario, there is no sharp 
sense in which this excitation is particle-like at the QCP. 